OPEN ACCESS

Edited by:

Lionel G. Nowak,

UMR5549 Centre de Recherche Cerveau et Cognition (CerCo), France

Reviewed by: Christian Casanova, Université de Montréal, Canada Roman Shusterman, University of Oregon, United States

*Correspondence: Chantal Milleret chantal.milleret@college-de-france.fr

Received: 14 June 2017 Accepted: 15 June 2018 Published: 18 July 2018

Citation:

Milleret C and Bui Quoc E (2018) Beyond Rehabilitation of Acuity, Ocular Alignment, and Binocularity in Infantile Strabismus.

Front. Syst. Neurosci. 12:29. doi: 10.3389/fnsys.2018.00029

\section{Beyond Rehabilitation of Acuity, Ocular Alignment, and Binocularity in Infantile Strabismus}

\author{
Chantal Milleret ${ }^{1 *}$ and Emmanuel Bui Quoc ${ }^{2}$ \\ ${ }^{1}$ Center for Interdisciplinary Research in Biology, Centre National de la Recherche Scientifique, College de France, INSERM, \\ PSL Research University, Paris, France, ${ }^{2}$ Department of Ophthalmology, Robert Debré University Hospital, Assistance \\ Publique - Hôpitaux de Paris, Paris, France
}

Infantile strabismus impairs the perception of all attributes of the visual scene. High spatial frequency components are no longer visible, leading to amblyopia. Binocularity is altered, leading to the loss of stereopsis. Spatial perception is impaired as well as detection of vertical orientation, the fastest movements, directions of movement, the highest contrasts and colors. Infantile strabismus also affects other vision-dependent processes such as control of postural stability. But presently, rehabilitative therapies for infantile strabismus by ophthalmologists, orthoptists and optometrists are restricted to preventing or curing amblyopia of the deviated eye, aligning the eyes and, whenever possible, preserving or restoring binocular vision during the critical period of development, i.e., before $\sim 10$ years of age. All the other impairments are thus ignored; whether they may recover after strabismus treatment even remains unknown. We argue here that medical and paramedical professionals may extend their present treatments of the perceptual losses associated with infantile strabismus. This hypothesis is based on findings from fundamental research on visual system organization of higher mammals in particular at the cortical level. In strabismic subjects (as in normal-seeing ones), information about all of the visual attributes converge, interact and are thus inter-dependent at multiple levels of encoding ranging from the single neuron to neuronal assemblies in visual cortex. Thus if the perception of one attribute is restored this may help to rehabilitate the perception of other attributes. Concomitantly, vision-dependent processes may also improve. This could occur spontaneously, but still should be assessed and validated. If not, medical and paramedical staff, in collaboration with neuroscientists, will have to break new ground in the field of therapies to help reorganize brain circuitry and promote more comprehensive functional recovery. Findings from fundamental research studies in both young and adult patients already support our hypothesis and are reviewed here. For example, presenting different contrasts to each eye of a strabismic patient during training sessions facilitates recovery of acuity in the amblyopic eye as well as of 3D perception. Recent data also demonstrate that visual recoveries in strabismic subjects improve postural stability. These findings form the basis for a roadmap for future research and clinical development to extend presently applied rehabilitative therapies for infantile strabismus.

Keywords: infantile strabismus, extending rehabilitation, cortical plasticity, interdisciplinary approach 


\section{INTRODUCTION AND OVERVIEW}

The visual scene may be decomposed into what are referred to as visual attributes, i.e., image location, orientations (horizontal, vertical, oblique), spatial frequencies (ranked from low to high, corresponding to gross to fine details respectively), velocities/directions of movement, binocularity (subtending $2 \mathrm{D}$ and 3D perception), contrasts and colors (Figure 1A). In infantile strabismus, i.e., strabismus occurring during childhood, the perception of each of these visual attributes can be altered as well are vision-dependent processes such as postural stability. But presently rehabilitative therapies by ophthalmologists, orthoptists and optometrists are restricted to preventing or curing only a few perceptual deficits among these. In the interest of helping these medical and paramedical practitioners evolve these therapies, we (i.e., a fundamental researcher and an ophthalmologist) hypothesize here that rehabilitation after infantile strabismus should be extended further, and we develop arguments in favor of such hypothesis.

This is pertinent since infantile strabismus occurs in $2-3 \%$ of children worldwide, and is a rather complex pathology occurring at a key period in the development of the visual system. Recall that strabismus is characterized by the two eyes not aligning simultaneously under normal conditions. One or both of the eyes may be deviated medially, laterally, upwards or downwards from the forward resting gaze position. The orientation shift may be constant or intermittent. Accordingly, the origins of these problems may be multiple, i.e., peripheral or central, sensory or motor, genetic or epigenetic (Bui Quoc and Milleret, 2014). Whatever the type and origin of such misalignment of the eyes, the symptoms first appear in childhood (Figure 2). When they appear in the first 2 years this is referred to as "early" infantile strabismus (early onset strabismus; $10 \%$ of the cases) while when they appear later than this, it is considered as "late" infantile strabismus ( $90 \%$ of the cases). In all cases, unfortunately, this corresponds to the peak of sensitivity of the "critical period" (or "sensitive period"), i.e., the time window when visual processing circuits of the growing brain (which are the neural bases for visual perception) have elevated plasticity and show heightened responsiveness to environmental influences (Hubel and Wiesel, 1970). In humans, considering together the processing of all of the diverse visual attributes, this period begins globally soon after birth, peaks between 3 months and 3 years (depending on the attribute) and terminates at about 10-12 years of age (Banks et al., 1975; Leguire et al., 1991; Epelbaum et al., 1993; Keech and Kutschke, 1995; Lewis and Maurer, 2005).

Consequently, the development of visual perception is altered in cases of infantile strabismus. The earlier the strabismus is the more important the visual perceptive alterations are. The development of perception of high spatial frequency components of images is severely affected because of the mismatch of information coming from the 2 eyes, which can lead to amblyopia, and hence to a loss in visual acuity. The development of binocularity and the resulting $3 D$ visual perception might also be altered. But it is less known that the development of perception of all the other attributes of the visual scene is also altered, including perception of image position, orientation, velocities/directions of movements, contrasts and colors (cf. Figure 1A). In other words, infantile strabismus leads to a general alteration of visual perception (Ho and Giaschi, 2006, 2009; Davis et al., 2008; Thompson et al., 2008; Husk et al., 2012; Husk and Hess, 2013; Li et al., 2015; Meier et al., 2016; cf. also Milleret, 1994; Kiorpes and McKee, 1999; Ho et al., 2006; Kiorpes, 2006; Hamm et al., 2014; for reviews). Visually-dependent processes are also affected in infantile strabismus. This is well illustrated when considering postural stability. Visionary medical practitioners were the first to detect this (Marucchi, 1987; Marucchi and Gagey, 1987) and it has been confirmed more recently by researchers in collaboration with medical and paramedical practitioners (Lions et al., 2014; Ezane et al., 2015).

Thus, infantile strabismus must be treated comprehensively. Otherwise, the above-mentioned losses may persist systematically. A three-step program is presently applied for this during the first post-natal years (when plasticity of the visual cortex is maximal) by ophthalmologists assisted by orthoptists and optometrists. This can eliminate, or at least limit, the negative consequences of infantile strabismus and to restore to normal impaired functions as much as possible. But unfortunately only a few visual attributes are treated in this program and their restoration is not always possible. First, optic corrections of refractive and/or accommodative errors of both eyes are performed. Then amblyopia of the "lazy" (deviated) eye is eliminated (or reduced or prevented) through occlusion of the "best" (non-deviated) eye. This helps restore the acuity balance between the two eyes as much as possible. Finally, the visual axes of the eyes can be realigned through surgery of the extraocular muscles and their tendons. This can facilitate binocular vision (and thus stereopsis). Gaze symmetry is also desirable for "esthetic" reasons. These goals are achieved in many but, unfortunately, not all, cases. This is because the medical and paramedical professionals have to deal with several difficult problems. First, as indicated above, infantile strabismus may have different origins and there are several types, with sometimes very complex combinations of symptoms. Second, each eye muscle does not work in isolation: rather the six extraocular muscles of each eye work in coordination with the others and with those of the other eye; thus they interact with one another through biomechanical and/or proprioceptive and/or brainstem and/or cortical mechanisms. A consequence of this is that when one or several extraocular muscle(s) is (are) operated, there is some impact on the others and this is not always predictable. Third, the motor activity of these extraocular muscles is under central influences that are not completely manageable by medical and paramedical professionals. Fourth, while rehabilitation of perceptual losses such as binocular vision is possible, this is strongly dependent on the timing of the occurrence of infantile strabismus (early vs. late onset). Thus, early onset infantile strabismus is, in general, considered to completely prevent the development of binocular vision and thus the development of stereoscopic vision. This is because in these cases the neuronal networks underlying binocularity have not yet developed in the brain before strabismus onset and may not develop later on (cf. Figure 2). Thus, whatever the post-natal age, neither eye surgery nor intramuscular injection of botulinum toxin may 


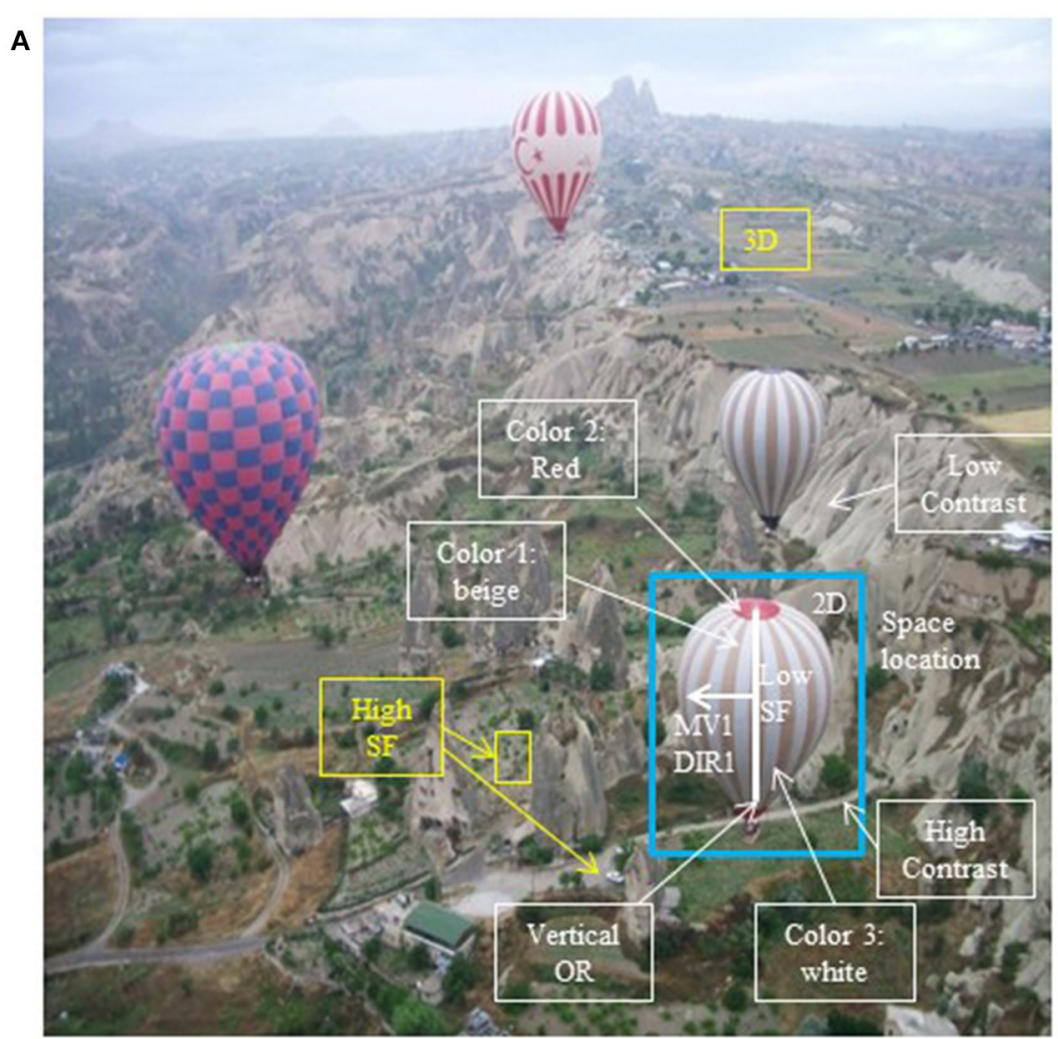

B

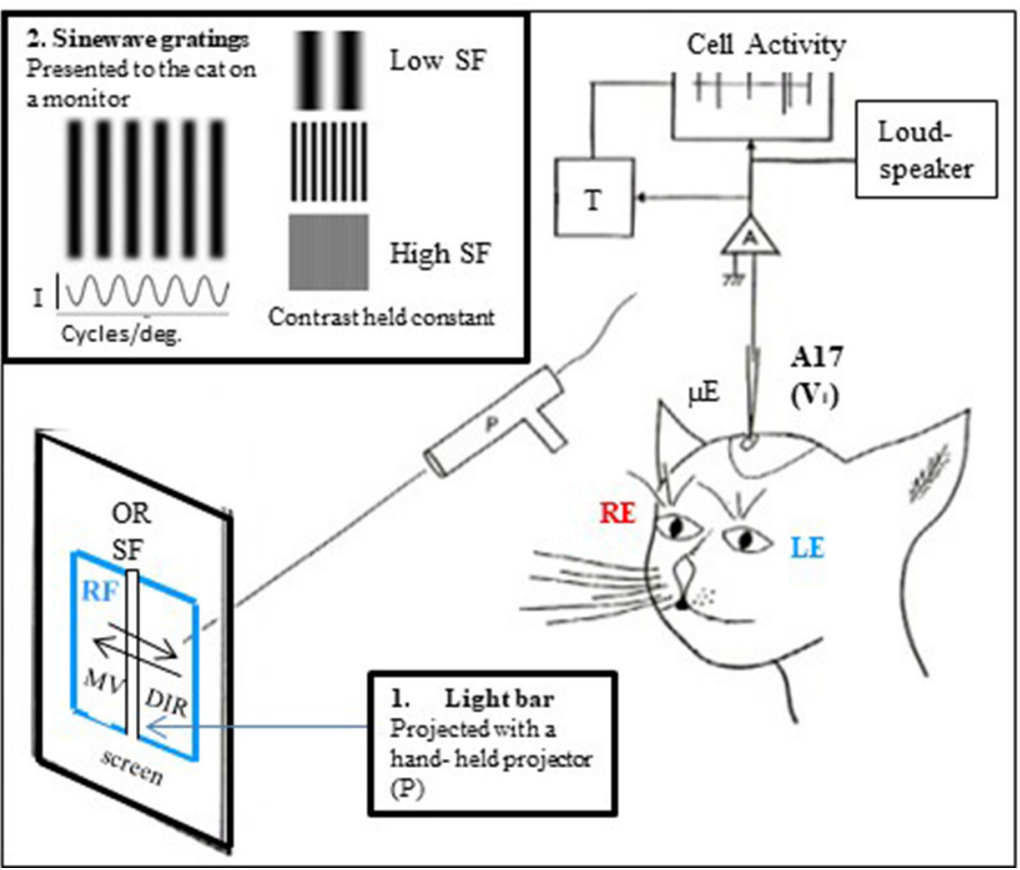

FIGURE 1 | The visual scene and its different visual attributes. (A) A real visual scene and its various attributes. As a general rule, the visual scene may be decomposed into the so-called visual attributes, i.e., space locations, edge orientations (ORs), spatial frequencies (SFs, ranked as low to high ones corresponding respectively to gross to fine details in the visual scene), movement velocities (MVs), directions of movement (DIRs), 2D and 3D perception subtended by binocularity, contrasts and colors. To illustrate this here, a photograph taken from a hot-air balloon flying over Cappadocia in Turkey has been analyzed: the nearest hot-air balloon (selected in the blue rectangle) is located in front, on the right ( $=3 \mathrm{D}$ and $2 \mathrm{D}$ localizations respectively). Its general orientation is vertical (vertical $\mathrm{OR}$, white line). The balloon displays large vertical stripes of equal width (= low SF) which are alternatively beige and white (colors 1 and 3); in contrast, its top and its basket are red (color 2). 
FIGURE 1 | This hot-air balloon is moving slowly $\left(\mathrm{MV}_{1}\right)$ toward the left $\left(\mathrm{DIR}_{1}\right)$. It is surrounding by 3 other hot-air balloons (= low $\mathrm{SFs}$ ) which are located at various distances from one another ( $3 \mathrm{D}$ localization). At this altitude, the bushes and cars on the ground are small (= high SFs). They are however easy to distinguish from surroundings (high contrast). On the other hand, the mountain slope appears uniform despite the presence of some heterogeneous elements (low contrast). The SFs and contrast are indeed tightly linked (Campbell and Maffei, 1981; see text). After infantile strabismus, the perception of all of these attributes is altered. But only the losses of high SFs and 3D perception (in yellow) are presently treated by medical professionals. (B) Cue attributes used during experiments performed in animal models to explore functionally the primary visual cortex. In a real visual scene, the visual attributes are varied, scattered and mixed (A) rendering it difficult to understand how each one specifically activates $V_{1}$ neurons. To solve this problem, Hubel and Wiesel $(1962,1965)$ positioned an anaesthetized, paralyzed cat in front of a screen oriented tangentially relative to the visual field. The screen projections were calibrated in degrees of the subject's visual angle. The area centralis (= foveas) and optic discs were also back-projected onto the screen to be able to determine the positions of the vertical and horizontal meridians onto the screen (Vakkur et al., 1963). Then the extracellular activity of single neurons was electrophysiologically recorded step by step (every 50 or $100 \mu \mathrm{m}$ ) in the different layers of the primary visual cortex (from layer I to layer VI) with a microelectrode $(\mu \mathrm{E})$ oriented perpendicularly or obliquely with respect to the cortical surface. The spikes generated by each recorded neuron were amplified (A), continuously visible onto an oscilloscope (cell activity), transformed into impulsions (T) and transmitted to a "Ioad-speaker." This allowed online identification of spontaneous or visually evoked changes of the neurons' activity. For each recorded neuron, the visual stimulus was a static or moving elongated (light or dark) bar manually projected (P) onto the tangent screen. Stimulus size and contrast were optimized by trial and error. The left eye (LE) and the right eye (RE) were systematically stimulated separately. Of particular interest here was the innovation of the use of a bar as a visual stimulus permitting for the first time to identify each neuron's receptive field (RF) to which it is sensitive. Then, still using such bar, they systematically characterized the visual attributes (except for colors) that best activated each neuron in $V_{1}$. These included the most effective orientation (OR), spatial frequency (SF), velocity and direction of movement (MV and DIR respectively) of the bar. The ocular dominance could also be determined by comparing visual responses to each eye individually. In more recent works, sine-wave gratings on a monitor placed in front of the animal were also used as visual stimuli for testing the respective attributes (e.g., Maffei et al., 1979; Albrecht et al., 1980; Albrecht and De Valois, 1981). An advantage of grating relative to bars is that the use of gratings also permitted to analyze precisely the neuronal responses to various $\mathrm{SFs}$, which values could be determined with great precision (in cycles/deg; see inset at top left). I, luminance intensity.

be effective (cf. Klainguti, 2005; but see Banks et al., 1975 who reported development of some binocularity in subjects with "early" onset infantile strabismus with very early corrective surgery). As a consequence, in most, if not all cases, early onset infantile strabismus patients will be limited to monocular vision for the rest of their life. In contrast, in cases of late onset infantile strabismus, the neuronal connectivity underlying binocular vision has had time to develop during the critical period before strabismus occurs. In effect, even if their vision is functionally altered by the strabismus, these patients can still recover stereopsis, provided however that the strabismus was properly managed.

Regrettably, nowadays the other visual perceptive losses mentioned above, including image localization, orientation discrimination, detection of velocities/direction of movement, contrasts, colors and the postural losses, are not taken into account in the rehabilitative therapy of infantile strabismics. Yet these losses are no less important than those that are currently treated. Beyond the tendency of medical and paramedical professionals to focus on monocular visual acuity and binocular vision, this neglect of other perceptual symptoms results from at least three other reasons. The first is the existence of some unavoidable limitations in the brain function that prevent any rehabilitation, whatever the medical action. For example, as evoked above, it is generally considered impossible to establish binocular vision and thus depth perception in a patient with early infantile strabismus. The second reason is our lack of knowledge. For example, perception of each attribute of the visual scene does not mature at the same age although their respective developmental timelines display clear overlaps (Bui Quoc and Milleret, 2014; Figure 2). Experimental data from higher mammals (cats, monkeys) and observations in humans have shown that each visual attribute has also its own critical period with its own time course, although they have not yet all been established (Wiesel and Hubel, 1963; Berman and Daw, 1977; Daw et al., 1978; Harwerth et al., 1986; Wang et al., 2010; see also Daw, 1998, 2009; Kiorpes, 2015; for reviews). In humans, at least to our knowledge, the time courses of only two critical periods are indeed presently known precisely. Both the critical periods for the development of human binocular vision and the one for visual acuity start very soon after birth and end rather late at $\sim 10-12$ years of age. But the former critical period peaks between 1 and 3 years of age (Banks et al., 1975) while the later has been reported to peak at $\sim 2$ post-natal years (Epelbaum et al., 1993). Note that in the latter paper the age when plasticity is reported as maximal is however likely imprecise because of difficulties in testing visual perception in infants under 2 years of age. Based on other methods, including clinical practice, this peak for visual acuity more likely occurs earlier at $\sim 3-6$ postnatal months (Leguire et al., 1991), thus earlier than the peak for binocularity. The third reason that perceptual symptoms resulting from infantile strabismus are neglected is the lack of therapeutically proven methods to rehabilitate perception of visual attributes other than high spatial frequencies (i.e., acuity) and binocular vision. As a consequence, overall, rehabilitation after infantile strabismus is presently rather limited.

Here, the main goal is thus to explore possibly more comprehensive approaches and whether solutions may be proposed to circumvent the current limitations. The final aim is to motivate and inspire new strategies to rehabilitate impaired perception of all (or almost all) of the visual attributes and facilitate recovered or at least improved function in "visuodependent" processes such as those which subtend postural stability. Our hypothesis is that this might be possible because of the organization and the functioning of the visual cortex which are overall governed by the same principles in subjects with normal vision and patients with infantile strabismus. One underlying principle is the convergence of information about the different attributes of the visual scene, in particular at the cortical levels (area $V_{1}$ and beyond), where global visual perception is elaborated. A second resulting principle is that of interactions and of interdependency of the various attributes of the visual scene during the elaboration of visual perception. Note that the visual system also has a substantial impact on the functioning 


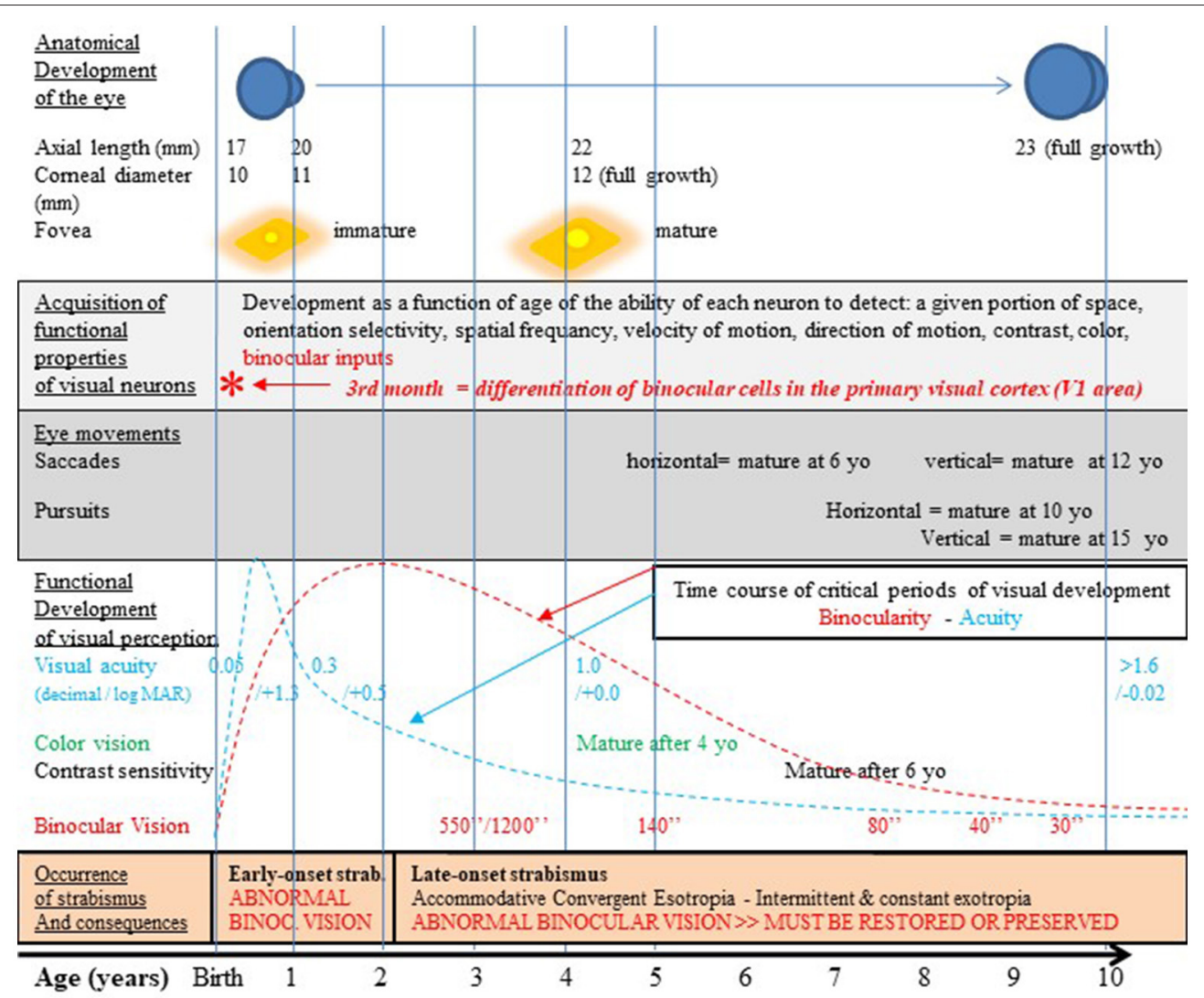

FIGURE 2 | Normal visual development in humans after birth and strabismus onset timings. The development of the visual system occurs first pre-natal and continues post-natal until at least 10-12 years as illustrated here. It includes the growth of the eye, an increase of the corneal diameter and the progressive formation of numerous and organized connections between the eyes and the cortex. This latter process at least occurs in concert with functional changes which are strongly vision-dependent. Thus, the retina matures, in particular within the fovea. Neurons in sub-cortical and cortical structures also acquire progressively adult functional characteristics. Among the latter processes, neurons in $V_{1}$ progressively acquire the capacity to be activated by stimuli of given positions in space and particular orientations, spatial frequencies, velocities and directions of movement, contrasts and colors. They also acquire binocular responses while they are initially mostly activated through the contralateral eye. In other words, cortical neurons progressively acquire specific selectivity for each visual attribute. Ocular movements such as saccades and pursuit also mature with age but not all at the same rate. Altogether, this leads to the development of visual perception including acuity, color vision, contrast sensitivity, binocular vision and 3D perception; this also leads to the development of space location, the ability to detect orientations and sensibility to movement (not indicated in the figure). All these processes occur during the so-called "critical period" of development which corresponds to a period of high plasticity with a peak during the first few post-natal years. Note that each visual attribute has its own critical period, with its own time course. For example, the critical period for acuity peaks at 3-6 PN months (blue curve; Epelbaum et al., 1993) while the one for binocular vision peaks later on at 1-3 years (red curve; Banks et al., 1975). Unfortunately, infantile strabismus (with early- or late-onset), which is associated to an abnormal post-natal visual experience, precisely occurs during these periods of very high plasticity (cf. the lowest part of the figure for details). The development of the anatomo-functional properties of the visual system and the development of visual perception of all the visual attributes may thus be greatly altered. But currently used treatments by medical and paramedical professionals and the strategy we propose in this paper may prevent, limit or eliminate such effects (cf. the text for further details). yo: years old; *, 3rd month: differentiation of binocular cells in the primary visual cortex. Reproduced from Figure 1 in Bui Quoc and Milleret (2014) with permission from Frontiers in Integrative Neuroscience and copyrights.

of other "vision-dependent" systems. Each of these principles is developed below. Also of interest here, considering the extensive adaptative potential of the CNS, is how these principles may apply at different stages of development, including adulthood. The plasticity of the visual cortex is indeed maximal during the post-natal critical period, from birth to $10-12$ years of age (as mentioned above) but some forms of plasticity still persist in adulthood (Milleret and Buser, 1984, 1993; Watroba et al., 2001; Baroncelli et al., 2011). After the detailed presentation of each of these principles, to support our hypothesis, a few examples will be provided for illustration. These will show the application of such principles and how they can guide new approaches through an inter-disciplinary approach to extending current therapies for rehabilitation of perception to multiple visual attributes and vision-dependent processes after infantile strabismus.

\section{PRINCIPLE OF CONVERGENCE IN VISUAL CORTEX}

\section{Convergence in Primary Visual Cortex}

In higher mammals with frontal normal vision, the different attributes of the visual scene are first processed in parallel (thus separately) within the primary visual pathway which includes 
the retina, the dorsal lateral geniculate nucleus located in the thalamus and the primary visual cortex (area $\mathrm{V}_{1}$, or A17).

The first evidence that parallel pathways in the mammalian visual system process different visual attributes came from electrophysiological recordings in the retina of the cat. EnrothCugell and Robson (1966) showed that a group of cells called $X$-ganglion cells respond to contrast and spatial frequency of an image. Others called Y-ganglion cells respond preferentially to moving stimuli. A third group of $W$-ganglion cells show still different (and very heterogeneous) functional characteristics (Wässle and Boycott, 1991 for review). Interestingly, these functionally distinct $\mathrm{X}, \mathrm{Y}$, and $\mathrm{W}$ ganglions cells correspond respectively to morphologically distinct $\beta, \alpha$, and $\gamma$ retinal ganglion cells whose proportional distributions are 45,5 , and $60 \%$ (Stone, 1983). These three classes of ganglion cells are at the origin of distinct pathways projecting differently through the dorsal lateral geniculate nucleus to the primary visual cortex which includes, in the cat, the three areas A17, 18, and A19 (cf. Payne and Peters, 2002 for details).

An equivalent organization was then found in primates (including humans). Three channels referred to as the parvocellular (P), magnocellular (M), and koniocellular (K) channels respectively ensure the processing of the different visual attributes. They correspond to the X, Y, and $\mathrm{W}$ channels in the cat respectively. As illustrated in Figure 3, each channel also originates from a distinct set of retinal ganglion cells ( $\mathrm{M}, \mathrm{P}$, and $\mathrm{K}$ respectively) which project in different manners to the dorsal lateral geniculate nucleus and then to $\mathrm{V}_{1}$ (corresponding to A17 only in primate). Livingstone and Hubel (1988) established that the $\mathrm{P}$ channel processes information relevant to the perception of form and color [red and green only, originating from the long-wavelength ("red") and the middle-wavelength ("green") cones], while the $\mathrm{M}$ channel processes information relevant to the perception of motion (originating mostly from the rods). Hendry and his collaborators then established that the $\mathrm{K}$ channel processes information relevant specifically to the perception of the color blue by originating strictly from the short-wavelength ("blue") cones; this information is however associated to "red-green" information, i.e., "yellow" at the cortical level (Hendry and Yoshioka, 1994; see also Hendry and Reid, 2000 for review). Note that the organization of the above described color channels is directly related to the fact that the perception of color originates from a comparison between "red" vs. "green" and "blue" vs. "yellow."

Nevertheless, whether in cat or primate, information processed through these different channels finally interact strongly in $V_{1}$. Each indeed differentially projects across the six cortical layers (I-VI) of $\mathrm{V}_{1}$ but numerous intra-cortical connections extensively inter-link these different layers both vertically and horizontally (Figure 3; see also Payne and Peters, 2002). Thus, as developed below, most neurons and most neuronal networks in $V_{1}$ can each be activated by most visual attributes, leading us to introduce here the notion of "convergence."

\section{Convergence at the Level of Single Neurons}

In their 1981 Nobel prize winning work, Hubel and Wiesel $(1962,1965)$ first showed with extracellular electrophysiological

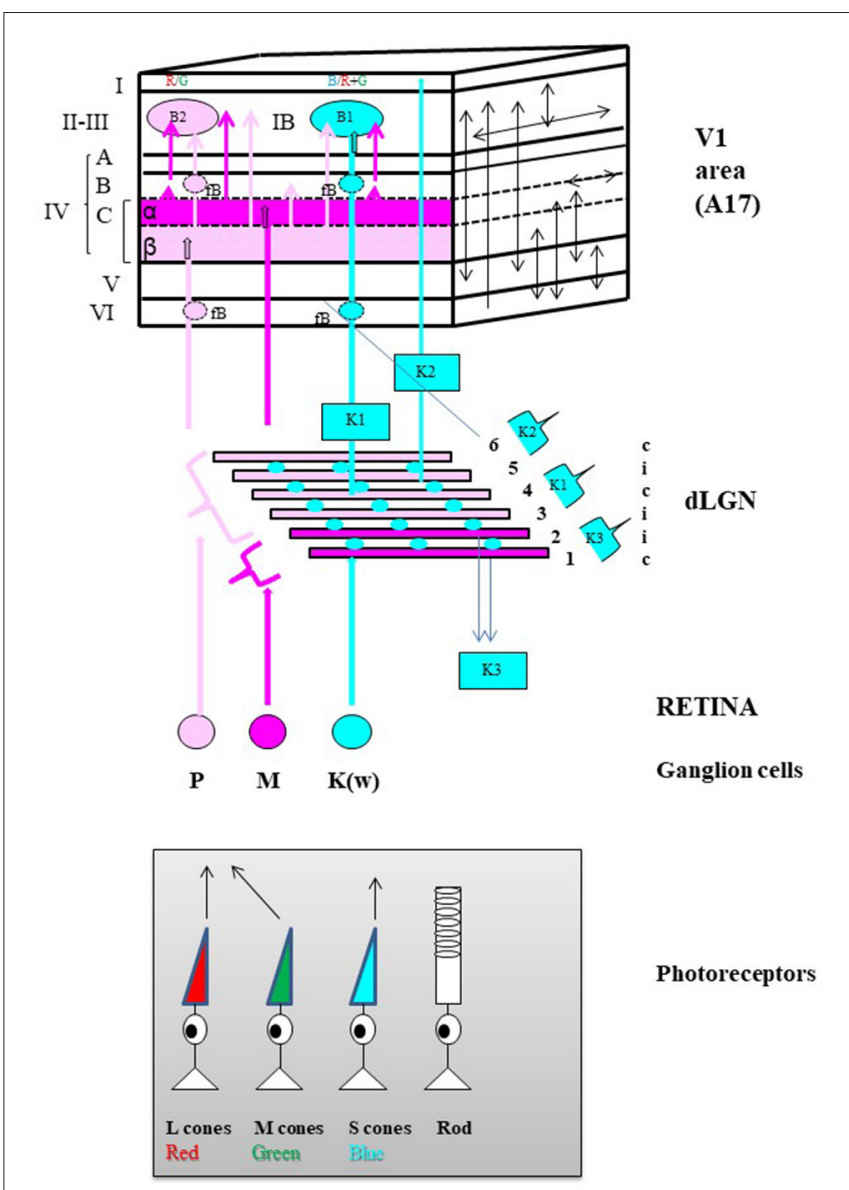

FIGURE 3 | General anatomo-functional organization of the primary visual pathway in primates. Main organization of the three "primary" parallel visual input channels. The primary visual pathway transmits $~ 90 \%$ of the retinal inputs to $V_{1}$. It is classically divided into three channels running in parallel designated respectively as magnocellular (M), parvocellular $(\mathrm{P})$ and koniocellular $\left(\mathrm{K}_{(\mathrm{W})}\right)$ which each process information about different subsets of visual attributes, but all respecting retinotopy, i.e., with receptive fields relative to their position in the retina. The $\mathrm{M}$ channel (in Magenta) mainly processes information relevant to the perception of motion, originating almost exclusively from rods located in the peripheral retina while the $\mathrm{P}$ channel (in light Pink) processes information relevant to the perception of form and colors (Red $[R]$ and Green [G] only), originating from the long-wavelength ( $L$, in red below) and the middle-wavelength ( $\mathrm{M}$, in green) sensitive cones mostly located in the central retina. Finally, the $\mathrm{K}$ channel processes information relevant to only the perception of the color blue since it originates strictly from the

short-wavelength ( $\mathrm{S}$, in blue) cones also mostly located in the central retina. Retinal processing develops some of the attribute characteristics of the respective channels which then leave the retina via axons of their $M, P$, and $\mathrm{K}_{(\mathrm{W})}$ retinal ganglion cells $(\mathrm{GG})$ respectively. Note that the latter group of GGs is referred to a " $K_{(W}$ " because it includes $G G$ cells which have not a specific name in the primate but which are similar in physiology and connectivity to the bi-stratified GG cells belonging to the W group in the cat retina (Hendry and Reid, 2000). Then the GG cell types of each eye project bilaterally to dedicated layers in the thalamic dorsal lateral geniculate nucleus (dLGN). M type GG cells project to the 2 deepest dLGN layers numbered 1 and 2 in the Figure (Magnocellular layers, in Magenta) while the $\mathrm{P}$ cells project to the 4 superficial layers numbered 3-6 (Parvocellular layers, in light Pink). Note the illustrated distributions from the contralateral (c) and ipsilateral (i) eyes. In contrast, the $\mathrm{K}_{(\mathrm{W})} \mathrm{GG}$ cells project to the inter-laminar regions of

the dLGN as intercalated layers (Blue circles). Finally, the respective dLGN layers

(Continued) 
FIGURE 3 | and inter-laminar regions have distinct projection patterns within $V_{1}$ while still respecting retinotopy along the cortical surface. Of the six main cellular layers of $V_{1}$ (in roman numerals), layer IV is the main target for geniculate inputs. It is composed of three different sub-layers: IV-A, IV-B, and IV-C with layer IV-C further sub-divided into sub-layers IV-C- $\alpha$ and IV-C- $\beta$. The $M$ retino-geniculo-cortical channel terminates in layer IV-C- $\alpha$ while the $P$ channel ends in layer IV-C- $\beta$. The main part of the $\mathrm{K}$ channel originates from the middle parts of the dLGN (indicated as "K1") and terminates in patches within layers II and III called "blobs" (B1, in Blue). Note that layers II and III are usually combined (as layer II-III) because they are difficult to be dissociated whether functionally or on the basis of histological material; also blobs may be rendered visible in histological preparations reacted for the energy metabolism marker cytochrome oxidase (not shown). A smaller projection also originates from the dorsal parts of the dLGN (indicated as "K2") and terminates in layer I. The final dLGN output pathway originates from the ventral parts of the dLGN (indicated as "K3") and projects outside $V_{1}$ to superior colliculus (Hendry and Reid, 2000). Numerous intracortical connections are then established both between the different cortical layers and within each cortical layer of $V_{1}$ (schematized as arrows on the plane at the right). For example, both the $\mathrm{M}$ and $\mathrm{P}$ channels project to all the blobs B1 and B2 (B2, see below) as well as outside the blobs (IB, inter-blob zone) of layer II-III. A few important notes about the organization of color processing: (a) By receiving information from both the $\mathrm{P}$ and $\mathrm{K}$ channels, the $\mathrm{B} 1$ blobs are dedicated to Blue/Yellow (B/) opponency, with $Y$ resulting from the combination of $R$ and $G$; (b) In contrast, by receiving information from the $P$ channel, the $B 2$ blobs are dedicated specifically to $R / G$ opponency. Thus there are two distinct processors of colors: $B / Y$ and R/G; (c) B2 (R/G opponency) blobs are 3 times more numerous than B1 (B/ $)$ blobs; (d) Neurons of the same color opponency seem to form vertical columns from layer II-III to layer VI, including the faint blobs (fB) of layers IV-B and VI; (e) Cells in the same type of color opponency blob have short intra-blob connections, and display correlated activities; (f) Cells belonging to adjacent blobs of the same type (i.e., which process the same color opponency) display correlated activities through short to long horizontal connections (e.g., Livingstone and Hubel, 1984a,b; Ts'o and Gilbert, 1988 for further details).

recordings that single neurons in $\mathrm{V}_{1}$ can be activated by several visual attributes of the visual scene. To do this they projected a static or moving elongated (light or dark) bar of optimal size (in terms of length and width) and optimal contrast onto a screen facing the animal (cf. Figure 1B for details) and tested each eye successively. They demonstrated that most $\mathrm{V}_{1}$ neurons respond selectively to the following attributes:

- A specific region of visual space. This region was generally rectangular and is referred to as the neuron's receptive field (RF in Figure 1B). Importantly, the neurons in $\mathrm{V}_{1}$ are organized by retinotopy, that is, along the surface of the cortex they are arranged according to the positions of their receptive fields in the retina. Note that a single neuron can have two overlapping receptive fields stimulated by the respective eyes.

- A preferred stimulus orientation which can be horizontal, vertical or oblique. This was shown by comparing responses to static bars projected $\mathrm{ON}$ and OFF onto the screen within the receptive field at various orientations. Two main types of orientation selective cells (with rectangular receptive fields) were identified in $\mathrm{V}_{1}$ (cf. Figure 4): (i) simple cells whose receptive fields displayed adjacent parallel regions responding respectively to ON and OFF visual stimulations. The orientation of these parallel regions defined the most effective orientation of the visual stimulus to activate the cell since the ON zones and the OFF ones are antagonistic; (ii) complex cells which displayed ON and OFF responses

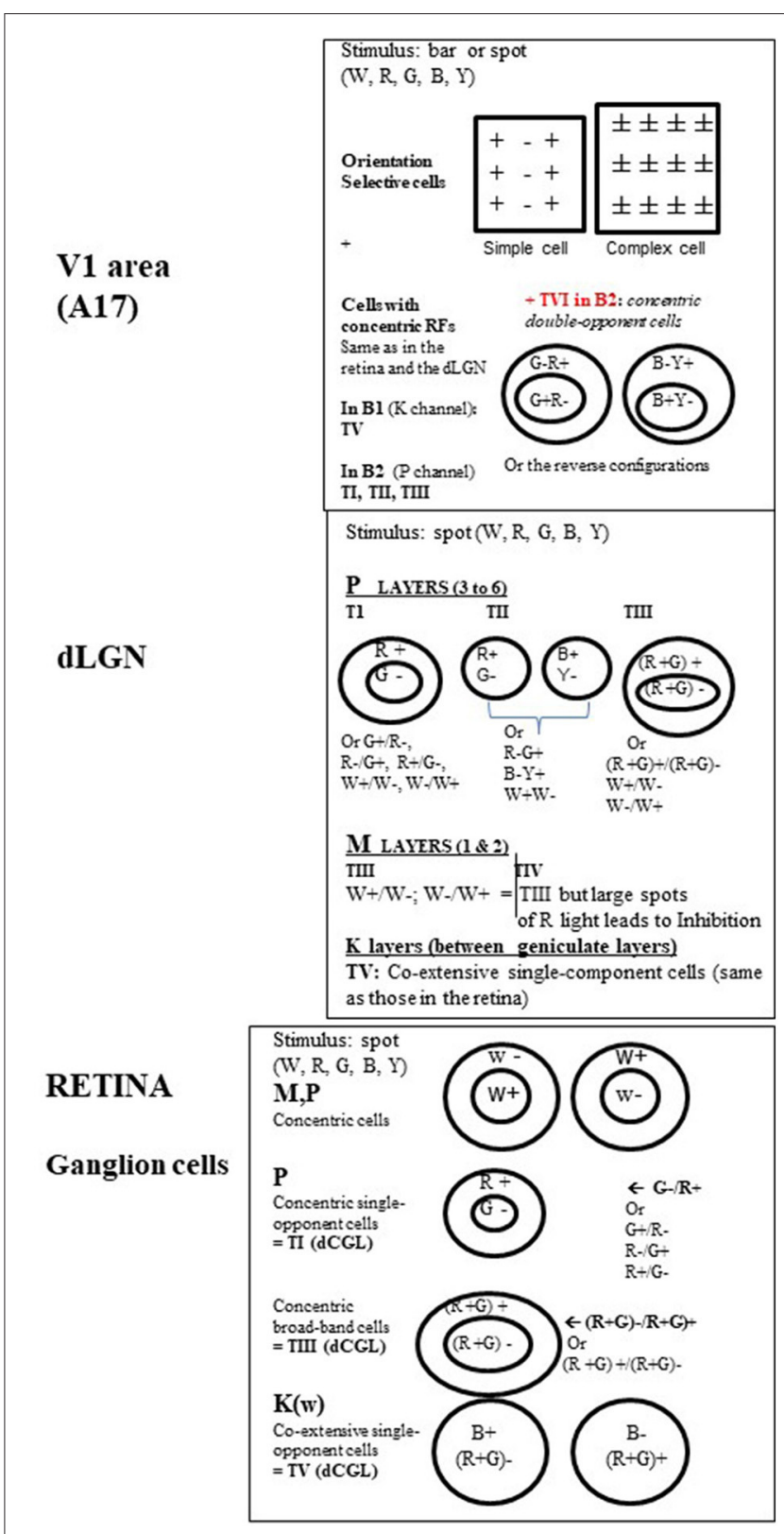

FIGURE 4 | Organization of the visual receptive fields (RFs) from retina to $V_{1}$ in the primate. Retina. In M, P, and K channels, the RFs of the ganglion cells (GGs) are all circular. This is shown using a stationary spot, the most effective stimulus at this level (Kuffler, 1953). In M and P channels, the GG RFs additionally respond to a light flashed "ON" at the center and "OFF" in the surround or display the reverse organization. In other words, they are of concentric center-surround opponent organization. But while M pathway GG cells only respond transiently to white (W) light (associated to motion detection), P pathway GG cells responses are sustained (i.e., last as long as the visual stimulus is present in the RF) and may be evoked by white (W), red $(R)$ and green $(G)$ light for both form and color detection. Two types of $P$ cells have been distinguished while activated by $R$ and $G$ light: (a) the concentric single-opponent cells ( $\mathrm{P}, 1$ st row) with for example a RF with center $\mathrm{G}$ - and a surround $\mathrm{R}+$ (see other possible combinations at right in the Figure). This is center-surround spatial and chromatic opponency; b) the concentric broad-band cells (P, 2nd row) for which, for example, the RF includes R and G both in the center and the surround but with opposite

actions. In the K channel (bottom row), the GG RFs do not display any concentric (Continued) 
FIGURE 4 | organization but display co-extensive single opponent responses, with opponent responses to blue (B) and Yellow $(\mathrm{Y})$ light, the latter resulting from $R$ and $G$ association (cf. Schwartz et al., 2000). They are evidently also implicated in color detection but they are the least numerous of the GGs. Note that the RFs associated with color perception also occur in the dLGN and were labeled types I, III, and $V\left(T_{\mid}, T_{\| I}\right.$, and $T_{V}$ respectively) by Wiesel and Hubel (1966). For convenience, this same nomenclature is applied here for the retina. dLGN. While the retina and the dLGN thus share some RF types, some new ones emerge here as well. Again, RFs are all circular but not all are of concentric organization. In the parvocellular layers $(P)$, in addition to types $T_{\text {I }}$ (the most frequent: 80\%) and $T_{|| l}$ ones, Wiesel and Hubel (1966) defined type $T_{\|}$. Contrasting with others, it exhibits opponent responses $(W+M$ - or $R / G$ or $\mathrm{B} / \mathrm{Y}$ ) but no center-surround arrangement and thus have been designated as single opponent cells. In the magnocellular layers $(M)$, beside type $T_{\text {III }}$ with solely $\mathrm{W}+\mathrm{W}$ - opponent between the center of the surround of the RF, is one type of color-sensitive RFs labeled as TIV RFs. It resembles $T_{\text {III }}$ RFs but large spots of $\mathrm{R}$ light produce a dominant and long-lasting inhibition of activity (Inh.). Considering that this latter type is "broad-band" with respect to color detection, and that only few cells were concerned, the role of the M pathway in color perception is considered to be rather limited. Finally, only $T_{V} R F s$ are in the inter-laminar K zone of the dLGN (Schwartz et al., 2000). On the basis of these characteristics, both the GG cells and the dLGN neurons are considered primarily to detect spatial position, luminance and contrast. In addition, $T_{l}$ and $T_{V}$ cells are specifically considered as "performing a sort of calculation that is necessary to disambiguate wavelength and intensity" and as "building blocks for color vision" (Conway, 2009). Note however that a few cells in both the retina and dLGN are better activated by stimuli of a given orientation (OR) and/or a particular direction of movement (DIR) of a visual stimulus (e.g., Soodak et al., 1987; Tailby et al., 2010; see also Wei and Feller, 2011; Vaney et al., 2012), characteristics principally found at the cortical level (see below). This indicates that, in spite of the segregation M, P, and $\mathrm{K}$ at these sub-cortical levels, a few neurons may be already activated by different visual attributes. $\mathbf{V}_{\mathbf{1}}$. In contrast with the retina and dLGN, most RFs in $V_{1}$ are rectangular in shape, are orientation selective and are better activated by a moving $W$ light bar of a given orientation. But stationary bars of a given orientation are also effective. Hubel and Wiesel $(1962,1965)$ first described these RFs as two main groups: simple, with alternating parallel regions responding respectively to ON and OFF light flashes (spot or bar), and complex which contrastingly display $\mathrm{ON}$ and OFF responses everywhere in their RFs. $\mathrm{V}_{1}$ cells are also activated by the respective visual attributes without color responses (except a few $C$ cells; cf. Table 1). The remaining $\mathrm{RF}_{S}$ in $\mathrm{V}_{1}$ recall those of the retina and the dLGN, being circular with concentric organization and responses to $W, R, G, B$, and $Y$ light (Livingstone and Hubel, 1984a; Ts'o and Gillbert, 1988). These include type $T_{\mid}$(concentric single-opponent cells), $T_{||}$(single opponent cells), $T_{\| I}$ (concentric broad-band cells), TIV (atypical concentric broad-band cells) and likely also TV ones (co-extensive single-component cells) RFs, with a specific distribution in the blobs B1 and B2 (cf. Figure 3). Another RF type found in B2 blobs are referred to here as Type $\mathrm{VI}\left(\mathrm{T}_{\mathrm{VI}}\right)$. These display center-surround RFs but each portion of the RF may respond to two colors $(R / G$ or $B / Y)$ and the response to a given color is reverse in sign in the center and the surround (cf. figure, on the top at right). In contrast with the other RF types, $T_{\mathrm{VI}}$ responds poorly, or not at all, to white light of any form, or to diffuse light at any wavelength. The corresponding cells are called concentric double-opponent cells. Although not numerous (5-10\% of $\mathrm{V}_{1}$ neurons), these cells are considered to underlie perception of local color contrast and color constancy (but not of color itself) by comparing color signals across visual space (e.g., Conway, 2009 for review). Thus they would contribute to perception of changes in the appearance of a color when contrasted with another one (for example, gray looks bluish if surrounded by yellow), and to make a color constant under very different viewing conditions. Globally, $V_{1}$ thus includes two distinct neuronal populations, those with rectangular RFs and others one with circular ones, with very different properties. See Table 1 for further details.

throughout their receptive fields. The orientation selectivity of these cells (whether simple or complex) was confirmed (and appeared even more clearly) by projecting the image of a moving bar of various orientations. Note that orientations at slight angular deviations from the most effective orientation also activated the recorded neuron but with decreasing efficacy which progressively diminished to zero when a certain angle was reached. This allowed them to define an angular range of orientation selectivity for each cell.

- A certain range of movement velocities. This was established by moving the image of the bar on the screen in a direction perpendicular to the orientation preferred by the neuron (Figure 1B). Orban et al. (1981) further demonstrated that neurons in cat primary visual cortex could be ranked into four main classes according to their movement response characteristics: "low pass" and "high pass" neurons responding respectively to only low or high speeds (up to $700^{\circ} / \mathrm{s}$ !), while "tuned" cells respond selectively to certain intermediate speeds and the "broad-band" cells respond to all speeds of movement of the visual stimulus.

- One or two opposite directions of movement. This was also established by moving the image of the bar perpendicular to the preferred orientation.

- The highest luminance contrast. This was assessed by studying the activity of each neuron in $\mathrm{V}_{1}$ while increasing the contrast between the luminance of the bar and the screen.

In addition, Hubel and Wiesel demonstrated that most ( $90 \%)$ neurons in cat $\mathrm{V}_{1}$ (A17) can be activated through both eyes, which strongly contrasts with geniculate neurons which are almost all activated by one eye (cf. Figure 3 ). In other words, most neurons in $V_{1}$ are normally binocular. This is very important here since pathways conveying all visual attributes converge from each eye onto virtually almost each cortical neuron. This thus leads to aberrant processing in cases of infantile strabismus because the bilateral ocular inputs do not correspond to the same part of the visual field and the precision of this convergence is fundamental for 3D perception (further discussed below).

These seminal studies have been confirmed and built upon by other observations, in various mammalian species including primates (e.g., Zeki, 1983; Hubel and Wiesel, 1988). Thus, for example, it has also been shown that:

- Most neurons in $\mathrm{V}_{1}$ are specifically activated by a larger portion of visual space than that described initially by Hubel and Wiesel $(1962,1965)$. With intracellular recordings (instead of extracellular ones), Bringuier et al. (1999) demonstrated that the visually evoked "synaptic integration field" of each neuron is in fact 6-8 times larger than previously thought. In other words, in addition to the "classical" receptive field established on the basis of spike activity, they found an additional surrounding zone in which changes of membrane potential of the neuron could be detected, but without evoking action potentials. This subthreshold activity was characterized by responses to flashed stimuli which decreased in strength and increased in latency at increasing distance from the "classical" receptive field (see also Frégnac et al., 1996 for review). Importantly in the present context, spikes from neurons recorded in $\mathrm{V}_{1}$ of cat have been detected in this same zone which is 6-8 times larger than the "classical" receptive field only a few weeks after monocular strabismus or monocular 
occlusion is induced in adulthood (Milleret and Buser, 1993; Watroba et al., 2001; Milleret et al., 2005). A likely explanation is that the "normal" sub-threshold activity may grow to become spiking activity as a compensatory consequence of the pathological viewing conditions. Supporting this, the same receptive field expansion was observed in cat $V_{1}$ after partial deafferentation of the retino-geniculo-cortical pathways through either optic chiasm section (Milleret and Buser, 1984) or retinal lesions (Gilbert et al., 1996; Abe et al., 2015).

- Most neurons in $\mathrm{V}_{1}$ are activated preferentially by a specific spatial frequency. This was demonstrated by combining extracellular recordings in $\mathrm{V}_{1}$ and the use of sine-wave gratings of various spatial frequencies as visual stimuli instead of a single bar (e.g., Maffei et al., 1979; Albrecht et al., 1980; Albrecht and De Valois, 1981). The sine-wave gratings were indeed pertinent since they included light and dark stripes which intensity changes gradually, in a sinusoidal fashion and their spatial frequency can thus be expressed easily and with precision as a number of cycles per degree of visual angle (cf. Figure 1B). Similar to other attributes such as orientation and velocity/direction of movement (see above), spatial frequencies near the preferred spatial frequency were less effective while very different spatial frequencies were ineffective.

- Most neurons in $\mathrm{V}_{1}$ display binocular disparity. This attribute is characteristic of binocularly activated neurons only, i.e., those neurons which display one receptive field for each eye (these receptive fields are located in the binocular visual field). The spatial overlap of the two receptive fields may be exact and thus binocular disparity would be nul. But, most often, receptive fields of a pair are rather separated very slightly $\left(<1^{\circ}\right)$, referred to as position disparity (which results from the retinal disparity of the image). Without going into detail, depending on the relative spatial position of the two receptive fields, this disparity may be crossed or uncrossed, depending on whether the target is located in front of or behind the fixation plane. This is also fundamental here since it contributes to elaborate depth perception at the cortical level (Ohzawa et al., 1990; DeAngelis et al., 1991; Trotter et al., 1992; Poggio, 1995; Anzai et al., 1997; Trotter and Celebrini, 1999; Durand et al., 2007; see also Poggio and Poggio, 1984; Trotter et al., 2004 for review).

However not all of the visual attributes converge onto all neurons of $\mathrm{V}_{1}$. Investigations performed in primates, in particular concerning the neural bases for color perception, lead to the conclusion that information about most visual attributes converges onto most neurons in $\mathrm{V}_{1}$ while the remaining neurons receive fewer inputs with diverse attributes. In other words, there are not one but rather two neuronal populations in $V_{1}$ (we shall call them Pop 1 and Pop 2 respectively) which differ both by their size and their degree of convergence.

These two neuronal populations are quite distinguishable when synthesizing, for example, data from Livingstone and Hubel (1984a) and those from Ts'o and Gilbert (1988), who precisely defined the responses to the different visual attributes (including color) of neurons recorded systematically within the different cortical layers of $\mathrm{V}_{1}$ in foveal and para-foveal regions of non-human primates (cf. Table 1). The largest neuronal population with the most convergence (Pop 1; panels in gray in Table 1) is found in the inter-blob zones (IB) of layer II-III, layer IV-B, and layer VI as well as in layer IV-C- $\alpha$ and layer V (cf. Figure 3 and legend for details). It includes strictly neurons with rectangular receptive fields (simple or complex; cf. Figure 4) which are activated specifically by orientation and velocity/direction of movement and which are also mostly binocular. However, these neurons are not selective for color (except in part IB of layer II-III, where a few complex cells have been found to be color selective). In contrast, the population with low convergence is smaller (Pop 2; panels without color in Table 1). It is restricted to the blobs of layers II-III, IV-B, and VI (cf. Figure 3 for details), layer IVC$\beta$ and a few cells in both layers V and VI. Its neurons only have circular RFs which are almost exclusively activated by colors (Types $T_{I}, T_{I I}, T_{I I I}, T_{V}$, and $T_{V I}$; see Figure 4 for details) and they are mostly monocular.

Considering the distribution of the $\mathrm{M}, \mathrm{P}$, and $\mathrm{K}$ channels in $V_{1}$ (cf. Figure 3), the greatest convergence of the different visual attributes occurs in neurons activated through the $M$ and/or the $\mathrm{P}$ channels (with the $\mathrm{P}$ channel being implicated in form perception). In contrast, the least convergence occurs in neurons mostly implicated in color perception, activated through the $\mathrm{P}$ and/or the $\mathrm{K}$ channels, which process respectively the antagonisms Red/Green and Blue/Yellow (cf. Figure 3).

In cases of infantile strabismus, as established again experimentally through electrophysiological recordings in $\mathrm{V}_{1}$ of animals (cats and non-human primates), both the Pop 1 and Pop 2 neuronal populations in $V_{1}$ still exist and remain different. The general organization of the visual system is indeed sustained. But responses to each visual attribute are altered in both populations (Pop 1: Chino et al., 1983; Kiorpes et al., 1998; Milleret and Houzel, 2001; Schmidt et al., 2004; Milleret et al., 2005; Watanabe et al., 2005; Bui Quoc et al., 2012; see also Von Noorden, 1978; Milleret, 1994; Wong, 2012 for reviews; Pop 2: Koçak-Altintas et al., 2000; Davis et al., 2008; Rajavi et al., 2015). Thus, in the Pop 1 neuronal population, each neuron still responds selectively to a specific region of visual space but some of them display larger receptive fields than in normal vision. About half of the neurons are still simple or complex (thus orientation selective) but neurons selectively activated by the vertical orientation may be rare or absent (in particular in case of infantile strabismus in the horizontal plane); the other half of neurons are not orientation selective. Also, neurons still respond best to a given spatial frequency but the low spatial frequencies become the most effective at the expense of high spatial frequencies; spatial frequency specificity is also generally lower than in normal vision (responding to a wider range of spatial frequencies). Altogether, the large receptive fields, the poor orientation selectivity and the poor spatial frequency selectivity observed in Pop 1 are the neural bases for amblyopia. Furthermore, most neurons now appear as monocularly driven through one eye or the other; only few binocular neurons 
TABLE 1 | Comparison of the degree of convergence of information about the various visual attributes of the visual scene onto neurons recorded at various depths in foveal and para-foveal regions of $V_{1}$ of primate macaque monkey (adapted from Livingstone and Hubel, 1984a; Ts'o and Gillbert, 1988).

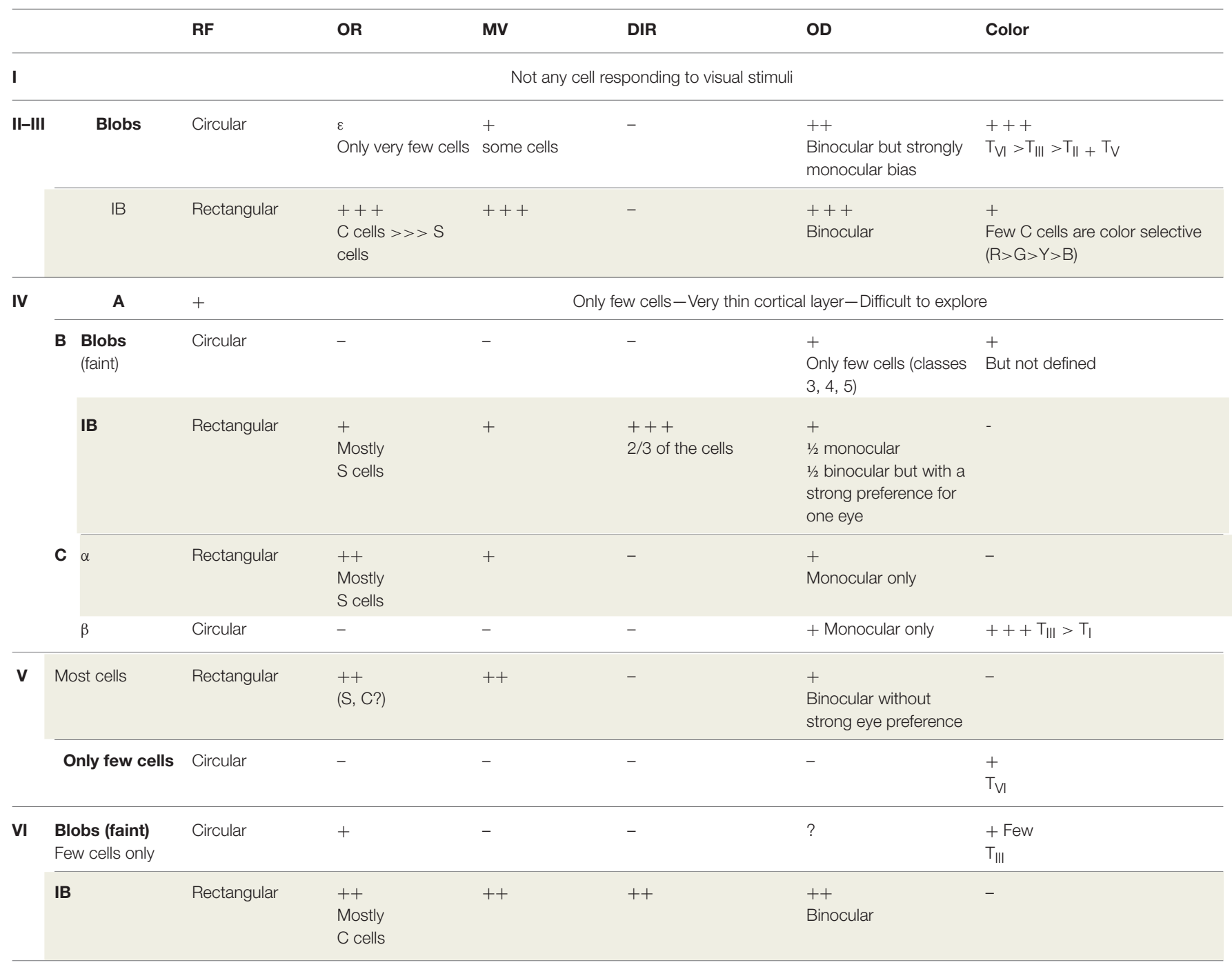

I-VI, cellular layers of $V_{1}$ from the most superficial to the deepest one; Blobs, mostly apparent in layer II-III but also slightly visible in layers IV-B and VI (Faint blobs); IB, inter-blob zone (cf. Figure 3 for details). Visual attributes considered here: RF, receptive field shape; OR, edge orientation; MV, movement velocity; DIR, direction of movement; OD, ocular dominance and color (Recall that cells selective for orientation OR are also selective for spatial frequency SF, not shown here). Analyzing the Table reveals that $V_{1}$ neurons may be ranked into two quite different populations. The first one (Pop 1), including strictly simple (S) and complex (C) cells with rectangular RFs, i.e., the most common cells in $V_{1}$, receives the greatest convergence of the various visual attributes information (in gray colored areas). They are distributed in IB of layers II-III, IV-B, and VI as well as in layers IV-C- $\alpha$ and V (in gray). By contrast the second one (Pop 2), located in blobs of layers II-III, IV-B, and VI as well as in layers IV-C-B and V (in non-colored areas), displays the smallest size and the smallest convergence. It indeed mainly includes monocularly activated cells with circular RFs of type $T_{l}, T_{I l}, T_{I I I}, T_{V}$, and $T_{V I}$ cells underlying color perception (cf. definition and specific locations in Figure 4).

may still be found. This is because, although still present, interactions between both eyes are altered. Without going into detail, this underlies the "interocular suppression" which helps avoid diplopia (Chino et al., 1994; Sengpiel et al., 1994, 2006; Sengpiel and Blakemore, 1996; Smith et al., 1997; discussed below). Binocular disparities are consequently completely abnormal because of the lack of binocularity and the inter-ocular suppression. The loss in binocularity and the impairment of binocular disparity are the neural bases for the poor (or even non-existent) depth perception. In addition, while neurons in strabismics still respond to a specific range of velocities of movement, the effective velocities of movement are slower than normal (meaning that the effective temporal frequencies are lower than normal), in particular in the periphery of the visual field. Although neurons remain selective for one or two directions of movement, the average directional bias of responses is significantly reduced. Note however that although eye movements of human may be asymmetric in case of early onset infantile strabismus, no prevalence of responding to naso-temporal directions of stimulus movement was found in animals in case of convergent or divergent infantile strabismus. Finally, the sensitivity to contrast also decreases markedly, whichever eye is visually activated. In the Pop 2 neuronal population, neurons retain their ability to respond rather 
specifically to color signal originating from one eye despite the pathology. But their responses to color are lower and slower than normal whether the amblyopic or the fixating eye is visually stimulated. Altogether, this indicates that the neural bases for the perception of all the visual attributes are altered after infantile strabismus.

Experimental approaches in animals have also allowed to establish that in case of infantile strabismus: (a) the more the central vision is concerned, the more the impairment is marked; (b) the more the deviation of the eyes develops near the peak of the critical period of a given attribute, the more the impairment is substantial (e.g., ocular dominance: Yinon, 1978; see also Figure 2); (c) there is no discernible relation between the degree of alteration of the neural bases for visual perception and the amplitude of the angle of deviation of the ocular axes after infantile strabismus (Yinon, 1978; Kalil et al., 1984); (d) M, P and $\mathrm{K}$ channels are all affected; (e) Among the above reported abnormalities, some at least likely result from the maintenance of functional immaturity in $V_{1}$ because of the misalignment of the eyes (for example large receptive fields, lack of orientation selectivity, poor ability to detect fast motions, etc...). Note that such observations were primarily made in experimental models of "early onset" strabismus in animals. This involves muscle surgery (in general by removing the external rectus) at an early age. Thus, it is rather a palsy that is created and it is not exactly similar to what occurs in human with innate infantile strabismus. But, the research community generally concurs that such data are applicable to humans with in particular an early onset strabismus, although the question of cause vs. effect must still be discussed (cf. Bui Quoc and Milleret, 2014 for details).

- Most neurons in $\mathrm{V}_{1}$ (belonging to the Pop 1 population) integrate information about most of the visual attributes, both in normally viewing and infantile strabismic human subjects. In other words, information about most attributes of the visual scene converges at the level of most single neurons in both viewing conditions.

- The remaining neurons in $V_{1}$ (belonging to the Pop 2 population) do not integrate as many types of attribute information as the Pop 1 population, whether subjects have normal sight or are strabismic since they have much less convergence of information of the respective attributes (in most cases, color and information from one eye only).

- In both populations, however, proximity between information from different visual attributes during visual perception may unavoidably favor interactions, an aspect which also interests us here (further discussed below).

\section{Convergence Within the Cortical Neuronal Networks}

As a general rule, each neuron in $V_{1}$ of higher mammals (such as cat and primate) functions as an integrated element of intracortical and/or interhemispheric neuronal networks which have both excitatory and inhibitory connections. This inspires the question of whether and how the principle of convergence described above might be extended to these neuronal networks. The following sections describe how this takes place both in subjects with normal vision and in infantile strabismus. What is possible because the neurons sharing the same properties are always preferentially interconnected whether considering single or several visual attribute(s).

\section{Anatomical organization of the neural networks of primary visual cortex}

Briefly, the neuronal networks in $\mathrm{V}_{1}$ of higher mammals with normal vision are composed of both vertical and horizontal cortical connections (cf. Figure 3 for a summary in the primate):

- Vertical connections form intra-cortical networks only. In primate, they originate mostly from layers IV-C- $\alpha$ and IV$\mathrm{C}-\beta$ which receive projections from the $\mathrm{M}$ and $\mathrm{P}$ channels respectively. Both project principally to layer II-III. From there, connections are established with all the other cortical layers through various vertical intracortical connections (e.g., Mitzdorf and Singer, 1978; Livingstone and Hubel, 1984a,b; Mitzdorf, 1985; Bolz and Gilbert, 1986; see also Gilbert, 1983; Payne and Peters, 2002 for reviews).

- Horizontal connections may be short or long-range intralaminar connections and thus may form intra- or interhemispheric networks. They mostly originate from layer IIIII although they may be present in all layers of $\mathrm{V}_{1}$ except apparently in layer I, and these are often reciprocal (e.g., Gilbert and Wiesel, 1983; Rockland and Lund, 1983; Houzel et al., 1994; Buzás et al., 2001; Rochefort et al., 2009 for details; see also Kisvárday, 2016 for review).

Globally, this organization of the cortical networks is sustained after infantile strabismus. But it includes abnormalities because the retino-geniculo-cortical pathway has developed some abnormalities first (Garraghty et al., 1989; Löwel, 1994; Li et al., 2011; Duan et al., 2015). Thus, for example, intracortical horizontal connections in cat primary visual cortex are modified (Schmidt and Löwel, 2008; see also below). Long-range interhemispheric callosal connections also develop asymmetrically between the hemispheres instead of symmetrically as in normal vision because of the stabilization of some juvenile callosal projections within the hemisphere ipsilateral to the deviated eye during post-natal development, projections which are normally eliminated (Innocenti and Frost, 1979; Lund and Mitchell, 1979; Bui Quoc et al., 2012). Of interest here, the above described data about callosal connections have been established in cats with early induced unilateral convergent strabismus. But similar data have been found recently in humans with spontaneous infantile strabismus (Ten Tusscher et al., 2018).

- Neurons in $\mathrm{V}_{1}$ are thus anatomically interconnected and form more or less extended neuronal networks both within and between the hemispheres. This holds true in subjects with normal vision or with infantile strabismus. This indicates that neuronal networks in both viewing conditions are organized in such a way that they may subtend the functional convergence which interests us here and this occurs within the whole extent of $\mathrm{V}_{1}$. 


\section{Functional organization of the neural networks in the primary visual cortex}

The principle of convergence also applies to the functioning neuronal networks in $\mathrm{V}_{1}$ of higher mammals. This is demonstrated here by first presenting the functional organization of the cortical neuronal networks implicated in the perception of a single attribute and then those underlying the perception of various attributes appearing simultaneously in the visual scene.

Functional organization of the neural networks implicated in the visual perception of one given attribute

(a) Subjects with normal vision

Those neurons of $\mathrm{V}_{1}$ which are activated by a particular attribute of the visual scene are first organized into columns oriented perpendicularly to the cortical surface and extending through all cortical layers (except layer I which is almost strictly composed of transversely connecting fibers). This constitutes a modular organization which is a general principle in the neocortex (cf. Mountcastle, 1997; for review). Hubel and Wiesel (1963a) again first showed this by using the same experimental protocol as described in Figure 1B. For example, they found orientation columns of neurons activated specifically by bars oriented at $45^{\circ}$ (see Figure 5A). They also showed that such orientation columns are surrounded by other columns selective for other orientations. Hence columns selective for a particular value (such as $45^{\circ}$ ) of a particular attribute (such as orientation) are scattered and thus display a discontinuous distribution throughout $\mathrm{V}_{1}$. Note that the numbers of columns for the respective values of an attribute (for orientation, this would be the various angles) are equal, thus preventing bias favoring any particular orientation. However, in most cases, neighboring columns include neurons activated specifically by nearby values of the attribute (such as orientation angles). A progressive shift in preferred attribute values thus occurs with distance so that all $360^{\circ}$ are covered by the network. This columnar organization applies to all other visual attributes except contrast (e.g., Hubel and Wiesel, 1962). Thus, for example, neurons recorded in a single vertical electrode track penetration in $\mathrm{V}_{1}$ may display similar ocular dominance. Of interest, all these columnar organizations in $\mathrm{V}_{1}$ are embedded in the retinotopic representation of visual space. Thus, neurons in each vertical electrode track include overlapping RFs (Figure 5A). But in contrast with the other visual attributes, the distribution of columns underlying the representation of visual space is continuous throughout $\mathrm{V}_{1}$. Note that such columnar organization are maintained after infantile strabismus in spite of the impairment of the functional properties of some neurons within these columns (e.g., Milleret and Houzel, 2001; Bui Quoc et al., 2012).

Second, the neuronal columns activated by a given visual attribute form a specific functional cortical map (Figures 5B,C). Considering the characteristics of each column, not surprisingly, each of these maps extend both radially over layers II-III to VI and horizontally over the whole surface of $\mathrm{V}_{1}$. The orientation and ocular dominance cortical maps were first visualized post-mortem
A

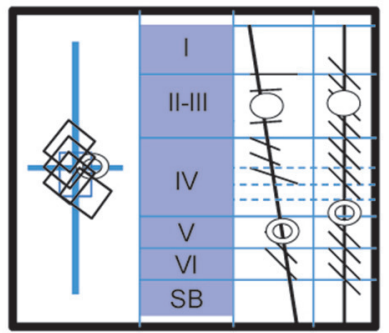

B

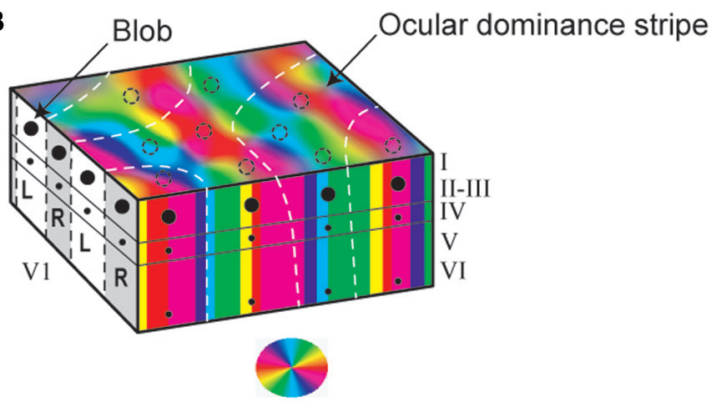

C

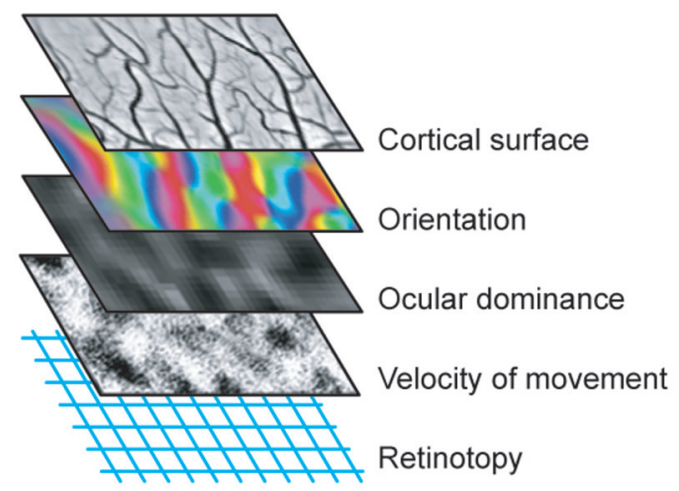

FIGURE 5 | Functional organization of $V_{1}$ neurons. (A) Columnar organization (demonstrated first by Hubel and Wiesel, 1963a with the protocol of Figure 1B). The visual responses evoked by neurons recorded in the respective oblique (3rd panel) or vertical (4th panel) electrode tracks were reconstructed from histological preparations, using as references the electrolytic lesions (represented as circles) applied through the microelectrodes at well determined depths at the end of each track. When the electrode penetration was vertical, thus perpendicular to the cortical surface, the neurons recorded in succession displayed overlapping receptive fields, i.e., were activated when overlapping and proximal portions of visual space were stimulated (rectangles in the left panel; vertical and horizontal blue lines correspond to the visual field meridians). Furthermore, all were activated by the same orientation (here diagonal) of the light bar (right two panels), thus demonstrating a columnar organization. In contrast, the oblique penetrations crossed various orientation columns and neurons successively lower in the same track were thus activated by different stimulus orientations rotating progressively from layer II-III through layer VI. Their receptive fields also gradually shift in the visual field (not shown). (B) From columns to cortical maps. Three distinct visual attributes (orientation, ocular dominance and color) serve here as examples to illustrate how columns of different attributes are organized to form maps in $V_{1}$. In the block of $V_{1}$ represented here, the vertical columns of neurons activated by the same orientation of the stimulus are represented by a given color according to the wheel shown below. Altogether they form a map which extends throughout the whole thickness of $V_{1}$ and which is visible from the cortical surface. Note that, within this map,

(Continued) 
FIGURE 5 | additionally, there are normally singularities where many different orientation columns abut, which are referred to as "pinwheels." They are not visible in the present orientation map but they resemble the color wheel below. Concerning ocular dominance, $L$ and $R$ indicate columns activated by the left and right eyes respectively (represented in white and gray respectively at left of the panel). In the superior part of the block of $\mathrm{V}_{1}$, these ocular dominance columns are represented here as forming a map with "stripes" (represented this time as white dashed lines). Note that the ocular dominance map is normally most easily detected in layer IV, where the geniculo-cortical afferents terminate and are still separate for each eye. In the other cortical layers, cells are indeed mostly binocular preventing the establishment of ocular dominance map (see text). But an ocular dominance map as illustrated here can be detected from any cortical layer (except layer I) after an abnormal post-natal visual experience such as in infantile strabismus since most cells are monocularly activated whatever their location (see text). Concerning color, contrasting with the two previous examples, each column is discontinuous since it is formed by vertically aligned blobs in layer II-III as well as faint blobs in layers IVB and VI (represented here as black spots at left and at front of the figure; cf. also Figure $\mathbf{3}$ and legend for details). However, they form a color map visible from the brain surface with various imaging techniques as illustrated by the dashed circles. These diverse cortical maps have specific geometrical relationships.

Thus, for example, the global ocular dominance map mostly crosses the global orientation map orthogonally. Also the blobs are mainly located in the center of the ocular dominance stripes. (C) Interlacing of cortical maps of different attributes in $V_{1}$. One cortical map per visual attribute exists in $V_{1}$ (except for contrast). Each may be visualized and characterized by optical imaging of intrinsic signals from the cortical surface following appropriate visual stimulations since each map extends through the $V_{1}$ layers in columns. Some of these attribute maps are represented here: orientation and ocular dominance (where black and white indicate ipsi- and contralateral dominance) as global cortical maps while the movement velocity map shows only one velocity selectivity as black points. Note that each has a unique distribution pattern. Here it is as if they overlap each other. But in fact they are interlaced. This permits all permutations of the respective attributes to be represented in a column for each part of visual space (also see text). Reproduced from Figure 2.3 in C. (Milleret, 2017), with permission from Elsevier Masson and copyrights.

by combining radioactive tracer injections and monocular visual stimulation (cat: Löwel and Singer, 1987; monkey: Hubel and Wiesel, 1969; Hubel et al., 1978; LeVay et al., 1985). Then, from the 90's until recently, cortical maps have been preferentially characterized both in cats and monkeys by using optical imaging of intrinsic signals (e.g., Bonhoeffer and Grinvald, 1991). Imaging of activity from the surface of the cortex of experimental animals exposed to visual cues thus established in vivo the global cortical maps for each visual attribute (retinotopy, orientation, spatial frequency, velocity/direction of movement, ocular dominance etc.) by visualizing the distribution along the cortical surface of the tops of the active columns (appearing as "patches," also called "domains") (see Figure 5C). To demonstrate, for example, the global orientation map in a surface activity imaging experiment, visual stimuli with eight different orientations (covering $360^{\circ}$ ) are first presented separately to establish the corresponding "single cortical maps"; then all the "single condition maps," each associated with a given color, are superimposed (Figures 5B,C). Note that, while the general organization is found in different individuals, the precise organization of the maps varies between individuals. The first global map that was visualized using such an imaging technique was of ocular dominance in primates. This was obtained by successive stimulations of each eye (Blasdel and Salama, 1986; Frostig et al., 1990; Ts'o et al., 1990; Grinvald et al., 1991). Many studies followed, establishing the global orientation cortical map, and showing its iso-orientation domains and singularities ("pinwheels"; cf. Figure 5B for illustration), with the respective orientation responses being arranged like the spokes of a wheel in which OR changes continuously around at the pinwheel center (Bonhoeffer and Grinvald, 1991, 1993a,b; Bonhoeffer et al., 1995; Maldonado et al., 1997; Shmuel and Grinvald, 2000; see also Schummers et al., 2002; Ohki et al., 2006). Up until recently, other functional global maps in $\mathrm{V}_{1}$ were characterized either with optical imaging or two-photon calcium imaging methods, including retinotopy (Bosking et al., 2000; Buzás et al., 2003; Schiessl and McLoughlin, 2003), spatial frequency (Issa et al., 2000; Nauhaus et al., 2012; Ribot et al., 2013, 2016), direction of movement (e.g., Bonhoeffer and Grinvald, 1993a; Shmuel and Grinvald, 1996; Kisvárday et al., 2000, 2001; Ohki et al., 2005), color (Livingstone and Hubel, 1984b; $\mathrm{Lu}$ and Roe, 2007, 2008), binocular disparity (Kara and Boyd, 2009) and temporal frequency (Yen et al., 2011). Not surprisingly, only the retinotopic global map was found to be organized continuously with "bands" of activity (corresponding to the various azimuths and elevations in the visual field) while the other global maps were found to be organized discontinuously (with "patches"). On the other hand, although neuronal activity in cortical maps of $\mathrm{V}_{1}$ clearly increases linearly with contrast, i.e., luminance (e.g., Lu and Roe, 2007), consistent with the absence of columns, no specific map has ever been found for this visual attribute: a contrast invariance was rather found over the whole extent of the cortex (cat: Carandini and Sengpiel, 2004, confirmed by Lu and Roe, 2007, in monkey). But a modular (thus discontinuous) representation of luminance polarity (ON or $\mathrm{OFF}$ ) has been found recently in layer IV of $\mathrm{V}_{1}$ (Smith et al., 2015a; Vidyasagar and Eysel, 2015; Kremkow et al., 2016), which receives thalamic afferent inputs (cf. Figure 3 and Table 1). Importantly, because this $\mathrm{ON}-\mathrm{OFF}$ organization originates from the clustering of $\mathrm{ON}$ and OFF thalamic afferents in $\mathrm{V}_{1}$, the authors propose that "all features of visual cortical topography, including orientation, direction of movement and retinal disparity, follow a common organizing principle that arranges thalamic axons with similar retinotopy and ON-OFF polarity in neighboring cortical regions" in $\mathrm{V}_{1}$. Note finally that subthreshold facilitation and suppressive surround maps, in correlation with "active" zone and "silent" surrounding zones of receptive fields (see above) were also found in cat visual cortex (Toth et al., 1996; Vanni and Casanova, 2013; see also below in section Principle of Interactions and of InterDependency of all the Attributes of the Visual Scene).

In the healthy subject, these global $V_{1}$ maps have several additional common important properties. Thus, the feed-forward retino-geniculo-cortical pathways as well as the intra-cortical and interhemispheric connections are organized congruently both anatomically and functionally, complementing one another. This ensures the convergence 
of information about each visual attribute in $\mathrm{V}_{1}$ in a coherent way (Bosking et al., 2000; Rochefort et al., 2007; Ribot et al., 2013). All the cortico-cortical connections (i.e., intra-cortical and interhemispheric ones) preferentially inter-link neurons with the same functional characteristics (e.g., Gilbert and Wiesel, 1989; Bosking et al., 1997; Rochefort et al., 2009) and thus ensure correlated activities within and between all the columns encoding for the same visual attribute in $\mathrm{V}_{1}$ of both hemispheres, for example those activated by the vertical orientation within the global orientation maps (Gray et al., 1989; Engel et al., 1991; Fries et al., 2002; see also Singer and Gray, 1995; Singer, 1999, 2013; Engel et al., 2001 for reviews). The mechanisms underlying functional architecture of $\mathrm{V}_{1}$ are so strong that the cortical representation of most visual attributes (thus the respective cortical maps) may emerge spontaneously, without any specific visual stimulation (Tsodyks et al., 1999; Kenet et al., 2003). Within a given global map, for example for orientation, activity changes within columns encoding for a certain orientation (for example vertical) through adapting (learning) processes may however lead to changes within the columns encoding for the other orientations over both short and long distances (cf. section Single Attributes). Each global cortical map (orientation, ocular dominance, direction of movement etc...) can be experimentally detected individually. For convenience, they are generally considered as if they overlap (Figure 5C). But, in fact, they are both anatomically and functionally tightly inter-linked in all layers of the visual cortex, which favors interactions (this underlies the main hypothesis advanced here).

(b) Patients with infantile strabismic

Of importance here, cortical maps are still present even in cases of infantile strabismus. The retinotopic, orientation, spatial frequency and ocular dominance maps have been described in experimental animals (e.g., Löwel et al., 1998; Bosking et al., 2000; Engelmann et al., 2002; Schmidt et al., 2004; Schmidt and Löwel, 2006a,b, 2008). They have also been observed in humans with infantile strabismus (e.g., Barnes et al., 2001; Goodyear et al., 2002; Clavagnier et al., 2015). This is not a surprise since most of them (except the direction of movement and the high spatial frequency maps) are genetically programmed and are thus present even without any visual experience (Milleret et al., 1988; Crair et al., 1998; Crowley and Katz, 1999; Li et al., 2006, 2008; Mitchell et al., 2009; Tani et al., 2012; Smith et al., 2015b). Functional specificity of feed-forward connections as well as long-range intrinsic and interhemispheric ones is also still present within these maps. Thus, for example, columns activated by the same orientation remain preferentially interconnected both within one given hemisphere and between the hemispheres (e.g., Schmidt et al., 1997). Correlating activities are also ensured through such connections between these columns (Roelfsema et al., 1994). But all of the cortical maps display abnormalities (whether observed in animals or humans) because their development depends on post-natal visual experience which has been incoherent and discordant because of the misalignment of the eyes.
Thus, for example, some columns within the maps are poorly activated, in particular when activated by the amblyopic eye (Goodyear et al., 2002; Schmidt et al., 2004). In correlation with this, some columns within the cortical maps are smaller than normal, such as those for ocular dominance, in particular when activated through the amblyopic eye (Löwel, 1994; Goodyear et al., 2002; Crawford and Harwerth, 2004). Because of interocular suppression, the excitation/inhibition balance within these maps is also disturbed (Sengpiel et al., 1994, 2006; see also Scholl et al., 2013). Some columns are discordantly paired through cortico-cortical connections. Thus, for example, in infantile strabismus, intra-cortical horizontal connections extend primarily between neurons activated by only one eye (instead of binocularly driven neurons in normal conditions) which indicates that fibers between coactive neurons (from one eye) are abnormally selectively stabilized (Schmidt et al., 1997; Schmidt and Löwel, 2008). Correlated activities between columns activated by the same attribute within each map are still present but they are also reduced, in particular those between different ocular dominance domains (i.e., those activated by the left eye and the right eye respectively) since binocularity is reduced (Roelfsema et al., 1994). But, of great importance here, all this is malleable, even in the adult (discussed below).

Functional organization of visual cortical networks underlying integrated perception of various attributes of the visual scene

(a) Subjects with normal vision

Considering the specific organization of both intra- and inter-hemispheric $\mathrm{V}_{1}$ neuronal networks implicated in the perception of the respective visual attributes (elaborated above), a key question then is whether and how the principle of convergence would apply there when several visual attributes are present, which is most often the case in natural environments. In fact, the principle of convergence applies in this situation despite its complexity, providing however the various inputs are coherent. This is indeed possible because: (i) each single neuron in $V_{1}$ can be specifically activated by several visual attributes (cf. section Convergence at the Level of Single Neurons). Thus, each one is also included in multiple cortical columns and several global cortical maps for the respective attributes; (ii) all of those neurons which are simultaneously activated by the exact same visual attributes, i.e., which display exactly the same functional properties, are included within the same columns as well as within the same parts of the global cortical maps; (iii) the assemblies of neurons sharing the same functional properties are always preferentially interconnected whether considering one single or several visual attributes. Thus, for example, the principle of convergence will be adhered to by all of the neurons in $\mathrm{V}_{1}$ whose receptive fields overlap at the center of the visual field and which are activated by a thin vertical bar moving slowly rightward while the right eye is visually stimulated. They will indeed all be located in the foveal representation of the retinotopic map of $\mathrm{V}_{1}$. They will also 
be included in 3 sets of columns located in this same cortical region activated respectively by the vertical orientation (in the global orientation map), movements at $5 \mathrm{deg} / \mathrm{s}$ to the right (in the global map of direction of movement) and the right eye (in the global ocular dominance map). The same applies for all neurons whose receptive fields overlap within the right hemifield at $0^{\circ}$ elevation and $30-40^{\circ}$ lateral of the vertical meridian, and which are activated by a horizontally oriented border moving at $100 \mathrm{deg} / \mathrm{s}$ upwards, detected through the left eye only, etc. The number of possible combinations is enormous; the principle of convergence may thus apply in $V_{1}$ to all sorts of neuronal networks activated by more or less numerous visual attributes, and implicated in the perception of more or less extended portions of the visual field. Of particular interest here, the principle of convergence thus also applies to extended neuronal networks in $\mathrm{V}_{1}$ while they are activated by various visual attributes present in large parts of the visual scene. Again, the preferred relations between the different columns activated by the same visual attribute within the cortical maps are implicated in this. But, additionally, both the long-range intra-cortical and inter-hemispheric connections will also contribute, allowing relations between extended and remote portions of $\mathrm{V}_{1}$. Thus, for example, interactions between regions $V_{1}$ encoding portions of space separated by several degrees become possible (Gilbert, 1983 for review). This is thought to be crucial for elaborating a global perception of the visual scene taking into account both the elements "of interest" in the visual scene but also the context (see below).

The principle of convergence does not apply here solely because neurons activated by the same visual attributes are preferentially inter-connected anatomically. Synchronization of oscillatory neuronal activity likely also plays a major role in this (cf. Milner, 1974; Von der Malsburg, 1981 for the theory). Wolf Singer and his collaborators were the first to support this view experimentally from their analyses of responses of single neurons in primary visual areas A17 and A18 of cats to single attributes. They indeed recorded synchronization of neuronal activity during the visual stimulation, mainly in the $\beta$ and $\gamma$ frequency ranges, i.e., $20-100 \mathrm{~Hz}$ (e.g., Gray et al., 1989; Engel et al., 1991; Fries et al., 2002; see also Singer and Gray, 1995; Singer, 1999, 2013; Engel et al., 2001; Fries, 2005; for reviews). These authors and many others further demonstrated that such oscillatory synchrony also applies to extended neuronal networks in visual cortex in general (i.e., in $\mathrm{V}_{1}$ and beyond) when activated by single visual attributes, in higher mammals including humans (Singer, 1999, 2013; Engel et al., 2001; Fries, 2005; for reviews). Very importantly here, in agreement with Milner and Von der Malsburg' theory (see above), these synchronizations are presently considered by Singer, his collaborators and many others to solve the "binding problem." That is, they assemble all of the attributes of a visual scene into a single coherent representation for visual perception. These synchronizations are even considered as being able to take into account cognitive factors such as the context, attentional state, etc. (e.g., Singer, 1999, 2013; Fries, 2005; for reviews). Synchronization of different zones has also been recently demonstrated to predict perceptual content (Hipp et al., 2011) and, when abnormal, to be involved in brain pathologies such as schizophrenia and autism (Uhlhaas and Singer, 2006; Uhlhaas et al., 2009a,b, 2011). However, the role of synchronization during the elaboration of visual perception should be confirmed or at least it should not be presented as universal in the present context. This is because this view has been challenged by many other authors. Thus, for example, Thiele and Stoner (2003) found that perceptual binding of two moving patterns had no effect on synchronization of the neurons responding to the two patterns. In the primary visual cortex, Dong et al. (2008) found that whether two neurons were responding to contours of the same shape or different shapes had no effect on neural synchrony. Revonsuo and Newman (1999) reported similar negative findings. Without going into details, a number of highly recognized researchers such as Shadlen and Movshon (1999) and Merker (2013) have also raised concerns.

(b) Patients with infantile strabismus

Considering the anatomical and functional organization of $\mathrm{V}_{1}$ in general, the principle of convergence may evidently also apply to visual neural networks of infantile strabismic patients underlying integrated perception of various attributes of the visual scene. However, not surprisingly, alterations in intracortical wiring and neural activity (e.g., Löwel and Singer, 1992; Schmidt and Löwel, 2008; see above for details) lead to reduced convergence and reduced neuronal synchrony in visual cortex relative to normal (Roelfsema et al., 1994). Recent data from the Hess team support this: they showed that interactions between cells in disparate brain regions are reduced when driven by the amblyopic eye of infantile strabismic subjects, from the dorsal lateral geniculate nucleus of the thalamus to higher visual areas, via $\mathrm{V}_{1}$ area (Li et al., 2011). They also demonstrated that amblyopia (in infantile strabismic patients) is associated to temporal synchrony deficits (Huang et al., 2012). This would then lead to altered visual perception, globally.

- These data indicate that the principle of convergence we have introduced and tested here applies to neuronal networks in $V_{1}$, whatever their size. Similar to single neurons, these networks are indeed also powerful integrators that are activated simultaneously by various attributes of the visual scene, providing, however, they work in a coherent way.

- This holds true both in normally viewing and strabismic people, although the principle of convergence is applied more comprehensively in normal vision.

These conclusions are the first two inescapable conditions to support our hypothesis that present treatments of infantile strabismus may be improved. Area $V_{1}$ indeed receives most $(\sim 90 \%)$ of the retinal inputs in higher mammals. And 
convergence needs to exit in it although it is mainly implicated in the decomposition of the visual scene in its basic features during the elaboration of visual perception (i.e., the so-called "segmentation" process).

\section{Convergence in Superior Visual Cortical Areas}

Here we focus on $V_{1}$ because it is activated by most of the retinal inputs (traveling through the geniculate pathway) in higher mammals and its anatomo-functional characteristics are rather well documented. But globally, there are more than 30 additional "superior" visual cortical areas in the so-called "visual cortex." In the present context, this raises again the questions of whether and how the principle of convergence may also be applicable to each of these cortical areas and to each neural network in which they are included. Among other arguments to justify these questions, one may evoke the fact that similar to $V_{1}$, each "superior" visual area includes neurons that are organized retinotopically, into "columns" and into "functional maps". But they are each implicated differently in the elaboration of visual perception (see Table 2 for details). Also each may belong to different neural networks (Figure 6). They are all activated (directly or not) via $V_{1}$ through "feed-forward" connections but each receives afferents from different other visual areas (Figure 6B). In most cases, each "superior" visual area also sends projections back through what are called "feed-back" connections but again these may project to different lower visual cortical areas, including $\mathrm{V}_{1}$ (Felleman and Van Essen, 1991). Beyond their specific corticocortical connections, some of these areas are also reciprocally interconnected with sub-cortical thalamic regions. As above, the amount of convergence will be compared successively in normally viewing subjects and those with infantile strabismus.

\section{Subjects With Normal Vision \\ Evaluation of convergence in each superior visual cortical area}

Most of the "superior" visual areas are listed in Table 2. For each, we have indicated the effective visual attributes and the resulting main functions. Thus, for example, the intra-parietal area (IP) displays a retinotopic organization and includes neurons which are activated by the orientation, the velocity/direction of movement and the position disparity of the visual stimuli. Accordingly, it contributes to extract the 3D shape and position of objects from $2 \mathrm{D}$ retinal images. As another example, $\mathrm{V}_{2}$ may be activated by almost all the visual attributes. It is mainly implicated in figure-ground organization, i.e., the tendency of the visual system to simplify a scene into the main object that we are looking at (the figure) and everything else that forms the background (or ground). In fact, various (but in general not all) attributes converge onto each of these superior visual areas. But the more the area is distant from $V_{1}$ (cf. Figure 6B), the more it contributes to the elaboration of visual perception in an increasingly complicated manner.

- The principle of convergence is respected within each of these superior visual cortical areas although fewer visual attributes converge on them compared to $\mathrm{V}_{1}$. This is coherent with the fact that each of these "superior" visual areas contribute specifically to the elaboration of visual perception (see also below).

\section{Cortico-cortical feed-forward connections}

The superior visual cortical areas may receive their inputs from $\mathrm{V}_{1}$ through two main pathways: either the dorsal or the ventral streams (Figure 6A; see Ungerleider and Haxby, 1994; Van Essen and Gallant, 1994; Shen et al., 1999 for reviews). Each includes different visual cortical areas which contribute to different aspects of the elaboration of visual perception (see just below for details). Whether and how the principle of convergence may still apply at the level of each of these streams again needs to be evaluated in the present context. Because of their complexity and limited space, only major properties and representative data are summarized below, with a few examples. More details including references are provided in Table 2.

The dorsal stream. extends from $\mathrm{V}_{1}$ to the posterior parietal lobe. It includes many visual areas such as $\mathrm{V}_{3}, \mathrm{~V}_{3 \mathrm{~A}}, \mathrm{MT} / \mathrm{V}_{5}$, MST $\left(\mathrm{V}_{5 \mathrm{~A}}\right)$, IP (sub-divided at least in MIP, LIP, VIP, AIP, CIP) and $\mathrm{PO} / \mathrm{V}_{6}$ which thus globally participate to more and more highly specialized and elaborated aspects of visual perception. Each area thus has specific functions which complement one another (cf. Figure 6B; see also Table 2 for details, including a key to these abbreviations). Thus, for example, the Middle Temporal area $\left(M T / V_{5}\right)$ is mostly specialized in analyzing motion (detection, direction and speed including gradients). It contributes to extracting $3 \mathrm{D}$ form information from $2 \mathrm{D}$ motion of an object. It plays a prominent role in the highlevel perceptual analysis of gestures, namely the construction of its visual representation. In addition, it is modulated by gaze direction and is activated during optokinetic stimulation. Complementing this, the Medial Superior Temporal area (MST) is mainly involved in the analysis of the movements of objects in space. It is also highly implicated in the perception of self-motion and optic flow (i.e., an apparent motion of visual space in the opposite direction to the movement of the subject). MST is also implicated in regulating pursuit eye movements and saccades. Both areas are consequently fundamental for the perception of movement including direction of movement, processing heading direction, and thus are involved in locomotion but each clearly contributes in a different way.

Altogether, the dorsal stream responds to the question "Where?" with respect to objects in space and thus is vital for spatial perception and spatial localization. It also helps respond to the question "How?" by dealing with visually guided action, that is, the subject's orienting movements relative to images localized in space. As summarized in Table 2, to ensure all these functions, the dorsal stream is more activated by the M (magnocellular) pathway. This is in agreement with the fact that it is the "movement" here that is mostly concerned. But it is also activated through the $\mathrm{P}$ (parvocellular) and $\mathrm{K}$ (koniocellular) pathways which provide additionally information about other visual attributes, i.e., the location in space, orientation, spatial frequency, ocular dominance, binocular disparity, depth perception, color and contrast. Information about hand and head position also have to be added. Since each 


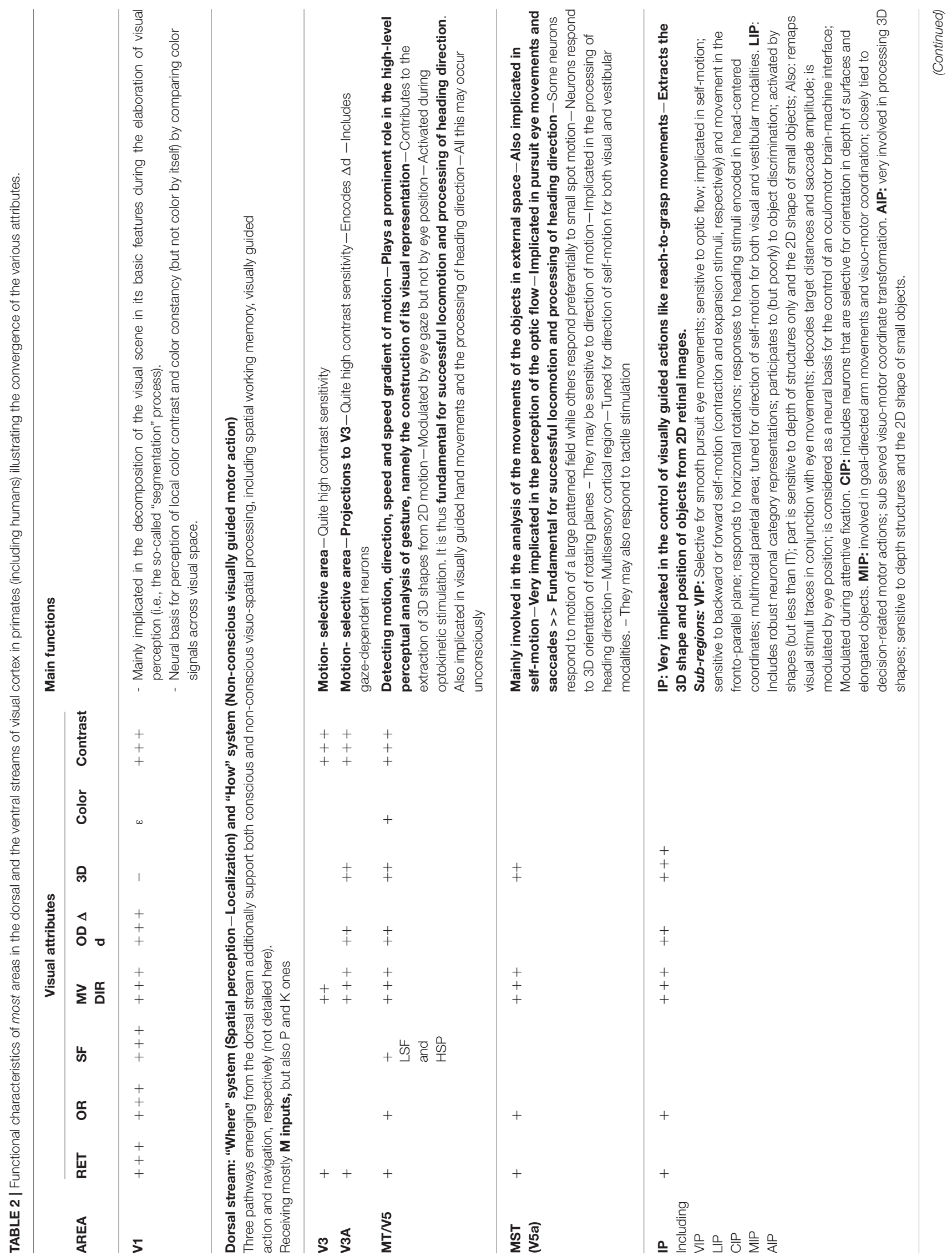




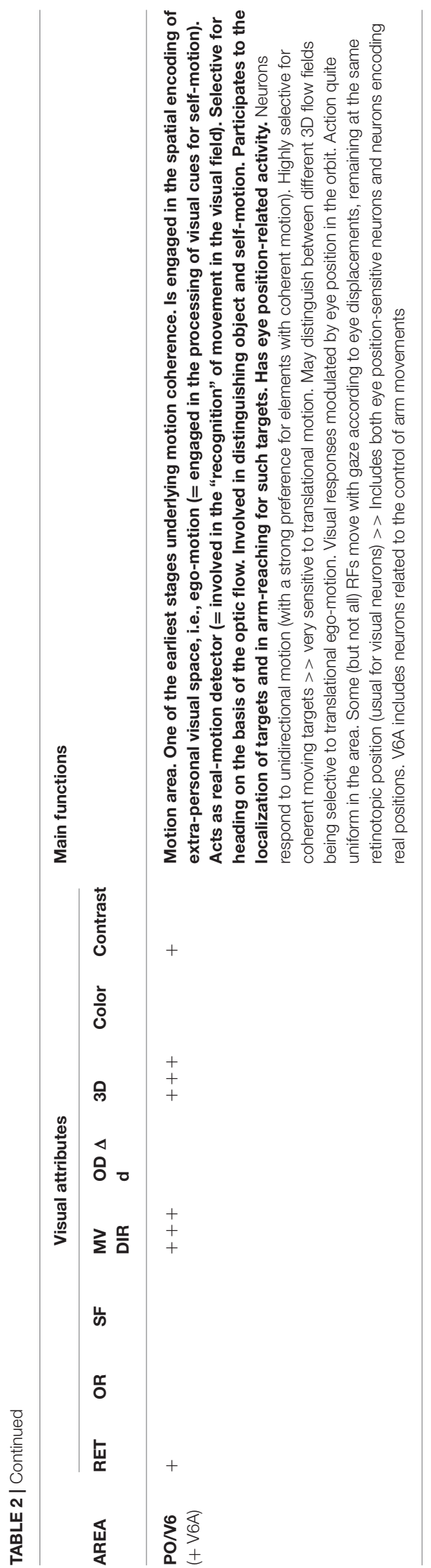

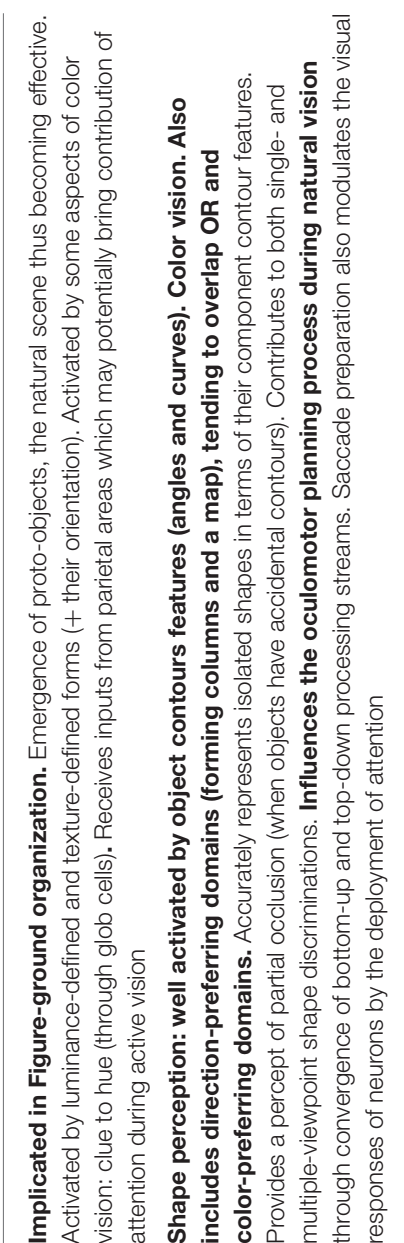

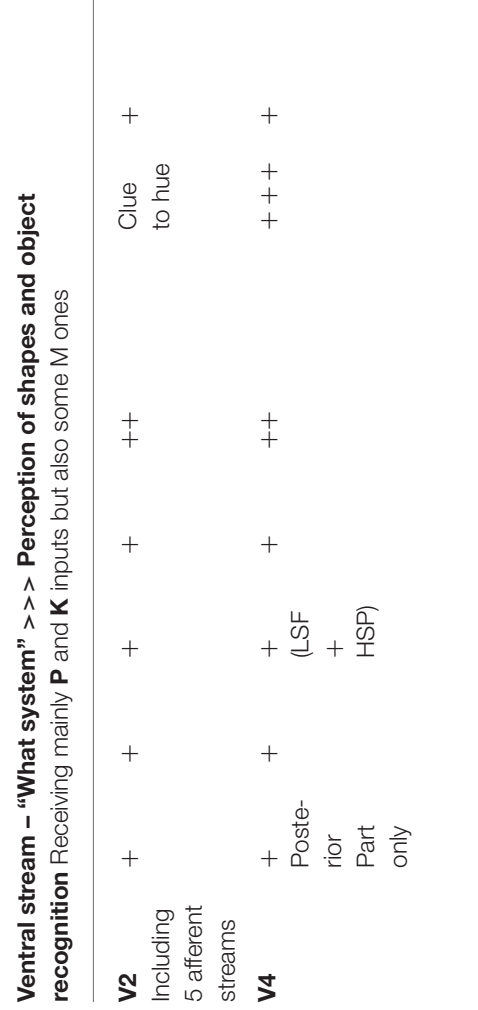

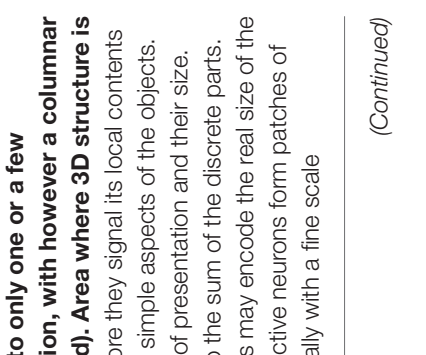

응 के क

\& ब

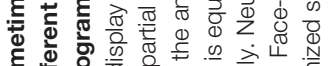

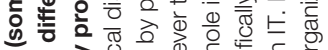
घ ॠ

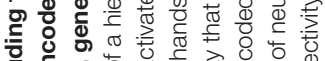

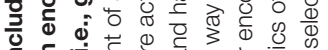

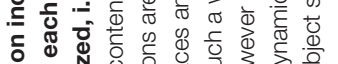
는 눈

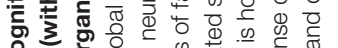

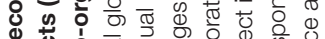

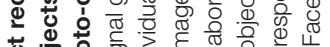

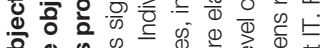

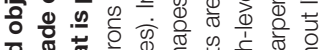

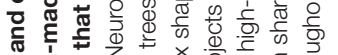

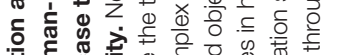

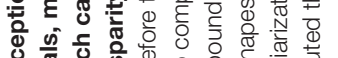

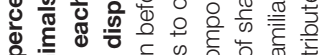
类.

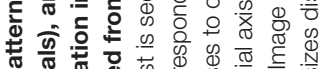

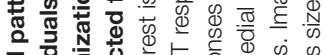

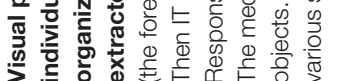

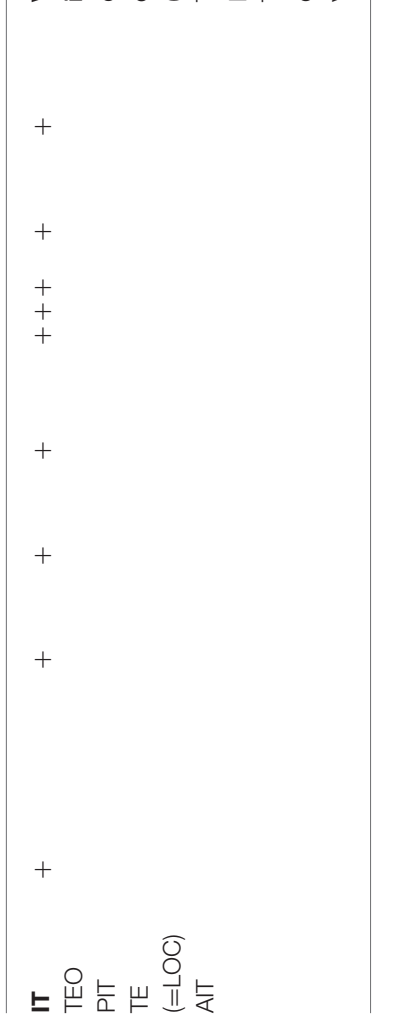




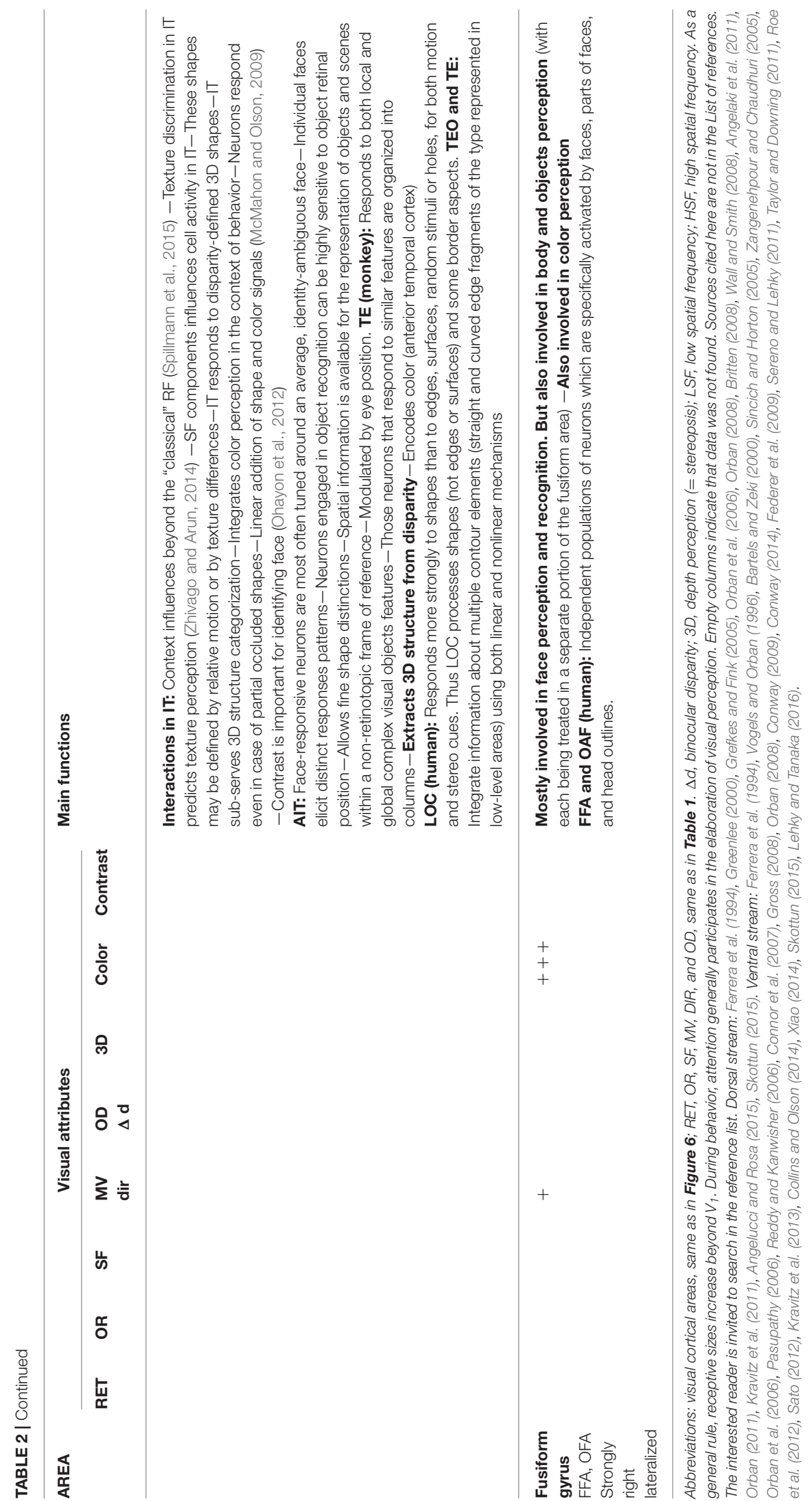


A

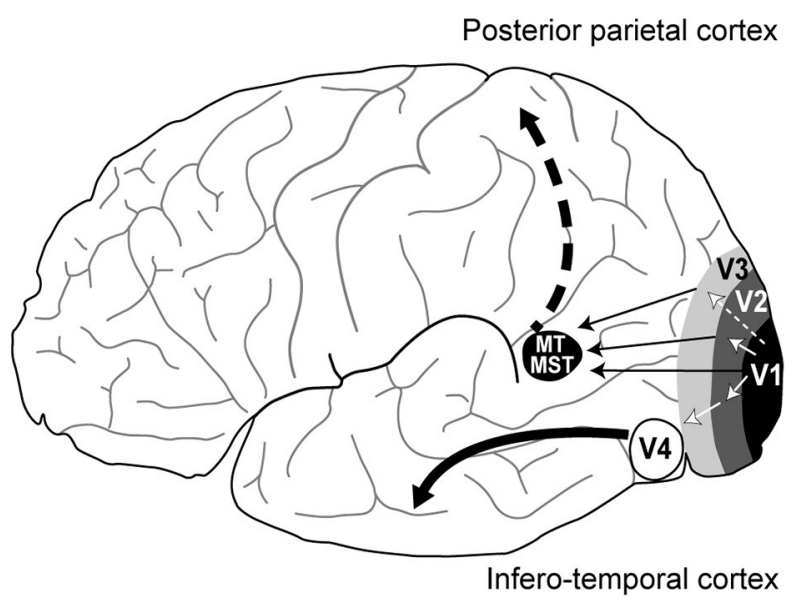

B

Parietal areas = "Dorsal stream"

Visual guidance and Action

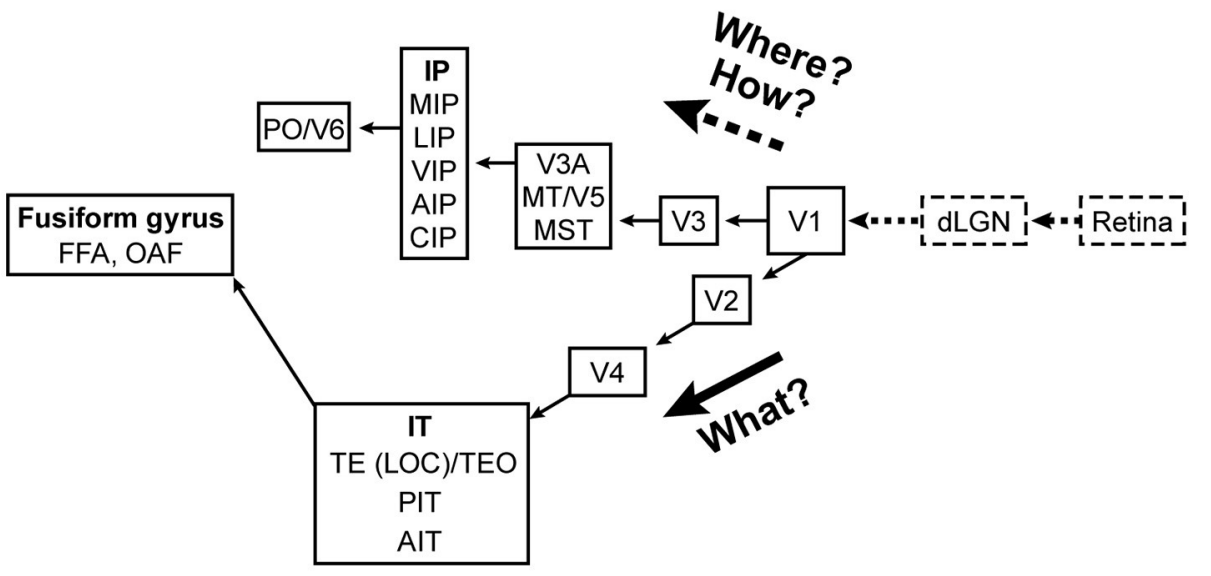

Occipito-temporal areas = "Ventral stream"

Identification of objects and the visual scenes

(recognition and categorization)

FIGURE 6 | Dorsal and ventral visual streams. (A) Brain localization in humans. Starting with $V_{1}$, the dorsal stream goes to posterior parietal cortex while the ventral stream goes to infero-temporal cortex. (B) The various areas included in the respective streams. Dorsal stream: $V_{3} ; V_{3 A} ; M_{T} N_{5}$, Middle Temporal area; MST, Medial Superior Temporal area; IP, intra-parietal area; MIP, medial intra-parietal area; LIP, lateral intra-parietal area; VIP, ventral intra-parietal area; AIP, anterior intra-parietal area; CIP, caudal part of the intra-parietal area; PO/N , parieto-occipital area; Ventral stream: IT, infero-temporal cortex; TE (=LOC)/TEO, Inferior Temporal Areas; PIT, posterior infero-temporal cortex; AIT, Anterior infero-temporal cortex; FFA, fusiform face area, OFA, occipital face area. Note that both streams interact with one another (not represented here, but see Table 5 and text for details). Reproduced from Figure 2.4 in C. Milleret (2017), with permission from Elsevier Masson and copyrights.

area included within this stream is activated differently by the different visual attributes (cf. section Evaluation of Convergence in Each Superior Visual Cortical Area), each is also differentially activated by the $\mathrm{M}, \mathrm{P}$, and $\mathrm{K}$ pathways.

- Thus, the principle of convergence of different attributes of the visual scene also applies to the dorsal stream of the visual system in higher mammals.
The ventral stream. runs from $\mathrm{V}_{1}$ to the infero-temporal lobe (Figure 6A). It includes various areas such as $\mathrm{V}_{2}, \mathrm{~V}_{4}$, IT (subdivided at least into TE (LOC)/TEO, PIT, and AIT regions) and the fusiform gyrus (including at least the FFA and the OAF areas) and thus is distinct from the dorsal stream. Similar to the latter, however, these areas globally participate to the elaboration of a more and more complex aspects of visual perception. Thus, for example, $V_{4}$ is activated by objects' contour features such as 
curves and angles. It contributes to both single- and multipleviewpoint based on shape discrimination. It is here that true color vision emerges, meaning for example that long and short wavelengths are now perceived respectively as "red" or "blue." But it also includes neurons preferentially activated by one given direction of movement and influences oculomotor planning processes. The Infero-Temporal area (IT) is one of the highest visual cortical areas. It is implicated in identification of faces, animals, man-made objects, etc. IT is also the visual area where $3 \mathrm{D}$ structure is extracted from binocular disparity. Both $\mathrm{V}_{4}$ and IT belong to the "What?" system since they are clearly implicated in the perception of shapes and recognition of objects.

As summarized in Table 2, to ensure these functions, the ventral stream mainly receives $\mathrm{P}$ and $\mathrm{K}$ inputs (for the elaboration of perception of shapes and color). But it also receives $\mathrm{M}$ inputs to encode movements. Again, information about various attributes of the visual scene are needed in order that each area in this stream carries out its own function. Since they differ from one area to the other, this indicates again that each is differentially activated through the $\mathrm{P}, \mathrm{K}$, and $\mathrm{M}$ pathways.

- The principle of convergence is thus also respected in the ventral stream, even if the processes are more complex than in $\mathrm{V}_{1}$.

\section{Cortico-cortical feed-back connections}

Higher visual cortical areas such as $V_{2}, V_{3}, V_{4}$, and $M T / V_{5}$ areas project back to $\mathrm{V}_{1}$. In the hierarchy of the cortical areas, the feed-forward connections are broadly defined as those that terminate mainly in layer IV (see Figure 3). In contrast, the feedback connections originate from neurons in superficial and/or deep layers and terminate mainly outside layer IV. The most superficial terminal fields exhibit a very precise "point-to-point" retinotopic connectivity while the deeper ones display a more diffuse organization (e.g., Batardiere et al., 1998; Markov et al., 2014). Some authors have even shown that the most superficial subset of feed-back connections terminate in a patchy fashion in layers II-III (Inter-blob zone), IV-B and V-VI of $\mathrm{V}_{1}$, and show modular and orientation specificity while the deepest ones are not only diffuse, but also unspecific and strictly terminating in layer IA (Angelucci et al., 2002; see also Angelucci and Bressloff, 2006 for review). Note that some exceptions to this rule have however been reported with some spatial overlap of feed-forward and feed-back terminals (Angelucci et al., 2002).

At least from our knowledge, the contributions of the feed-back connections during the global elaboration of visual perception are fundamental but complex and far from being completely understood. For example, Gilbert and Li wrote that such connections likely "carry rich and varied information about behavioral context, including attention, expectation, perceptual tasks, working memory and motor commands" (Gilbert and Li, 2013), but this remains to be clarified. On the basis of various studies however, such connections are at least recognized as contributing to refine and modulate neural activity in the lower structures. This has been demonstrated in $\mathrm{V}_{1}$ unit recordings, where feed-forward intra-cortical horizontal connections and feed-back connections from superior visual cortical areas play complementary roles during the elaboration of visual perception (e.g., Stettler et al., 2002; Schwabe et al., 2006; Piëch et al., 2013; Ramalingam et al., 2013; discussed further below).

Altogether, the feed-back connections likely contribute to perceptual grouping of the elements of a single object that are represented in a fragmented manner by neurons with limited receptive fields. This includes contour integration, distinguishing contextual interactions and perceptual segregation of overlapping objects, including perceptual discrimination accuracy. Their implication in perceptual grouping and contextual interactions at least evidently implies that such feed-back connections necessarily favor a coherent convergence of information within $\mathrm{V}_{1}$ area during the elaboration of visual perception, at least by some aspects.

- Thus, for coherence, even if this question has not been investigated specifically yet, our guess here is that the principle of convergence needs to be also respected by the feed-back connections.

\section{Trans-thalamic cortical routes}

The superior visual cortical areas are also reciprocally connected with various sub-cortical thalamic regions belonging to the extra-geniculate nuclei complex (e.g., thalamo-cortical: Berman and Wurtz, 2011; Gamberini et al., 2016; cortico-thalamic: Ungerleider et al., 1984; Van Essen, 2005). Such trans-thalamic cortical routes also contribute in some ways to the elaboration of visual perception. Thus whether and how the principle of convergence may still apply at their level needs to be examined as well. We cannot analyze each of these trans-thalamic cortical routes here. Anyway, most of them are not totally characterized. Thus, to approach this question, we have rather decided to focus on the representative route formed by $\mathrm{MT} / \mathrm{V}_{5}$ and its reciprocal connections with the pulvinar nucleus located within the thalamus (e.g., Ungerleider et al., 1984; Shipp, 2001; Berman and Wurtz, 2008; for review, see Casanova, 2004). Both of these structures are strongly implicated in higher-order motion integration $\left(\mathrm{MT} / \mathrm{V}_{5}\right.$ : cf. section Cortico-Cortical Feed-Forward Connections and Table 2 for details; pulvinar nucleus: Villeneuve et al., 2005; see also Merabet et al., 1998; Casanova et al., 2001; Dumbrava et al., 2001).

As shown above, $\mathrm{MT} / \mathrm{V}_{5}$ already benefits from considerable convergence through the dorsal stream although it is mostly activated via the $\mathrm{M}$ pathway (cf. section Cortico-Cortical FeedForward Connections and Table 2 for details). The same holds true for the pulvinar nucleus which is even considered as a "connectional hub" and a major "relay" between the thalamus and the cortex (Bridge et al., 2016). The convergence of abundant visual information is more especially ensured in the pulvinar nucleus by its reciprocal connections with many superior visual cortical areas including $\mathrm{V}_{2}, \mathrm{~V}_{3}, \mathrm{~V}_{3 \mathrm{~A}}, \mathrm{~V}_{4}, \mathrm{MST}$, and FST, in addition to $\mathrm{MT} / \mathrm{V}_{5}$, belonging to both the dorsal and the ventral streams (e.g., Grieve et al., 2000; Van Essen, 2005; Arcaro et al., 2015; for review, see for example Bridge et al., 2016). Thus the pulvinar nucleus likely receives information from all three visual pathways ( $\mathrm{M}, \mathrm{P}$, and $\mathrm{K}$ ) through these reciprocal cortico-thalamic connections and integrates all of the visual attributes, even if 
it has been more implicated in motion perception. Note that, in complement, the pulvinar nucleus receives retinal afferents through the extra-geniculate pathway, originating at the level of the optic tracts. These afferents project from the retina either directly or indirectly through the Superior Colliculus (located in the midbrain, also partially implicated in vision) to different parts of the inferior pulvinar nucleus, with the indirect ones being likely dominant (e.g., Berman and Wurtz, 2011; Bridge et al., 2016; Kwan et al., 2018). In agreement with the visual information being exchanged between the pulvinar nucleus and the visual cortex (see above), these retinal fibers apparently comprise axons of all three major ganglion cell classes, i.e., at the origin of the M, P, and K pathways (e.g., Cowey et al., 1994).

Altogether, these data indicate that the principle of convergence may also be respected within the trans-thalamic cortical routes. On the basis of the above described example, this convergence is even likely very extensive suggesting an implication of these routes in many (but not yet completely identified) situations.

\section{Infantile Strabismic Patients}

Consistent with functional changes in $\mathrm{V}_{1}$, superior visual cortical areas of both the dorsal and ventral streams are also affected after infantile strabismus (e.g., Thompson et al., 2012 who conducted their study in humans). Thus, for example, it has been established that global and local perception of forms, including faces, is altered; both global and the local motion perception is altered as well (e.g., Birch, 2013; Hamm et al., 2014; Levi et al., 2015 for reviews). But also, similar to $\mathrm{V}_{1}$, their general anatomo-functional organization remains unperturbed since it mainly results from innate and genetic factors. Note that although little is known about changes in feed-back connections and trans-thalamic cortical routes in infantile strabismus, our guess is that the same applies to them. One may underline however that Thompson et al. recently reported unexpected but very interesting findings concerning the latter connections, more specifically the ones between $\mathrm{MT} / \mathrm{V}_{5}$ and the pulvinar nucleus (cf. section Trans-thalamic Cortical Routes). In the light of previous work indicating a functional $M T / V_{5}$ deficit in case of amblyopia, they first conducted a psychophysical experiment investigating the ability of amblyopic eyes to perceive coherent and incoherent plaid stimuli (Thompson et al., 2008). To their surprise, however, they found that amblyopic eyes mediated largely normal perception of both coherent and incoherent plaids. They thus proposed two possible (nonexclusive) explanations for this finding. The first was that the integration of local motion signals in $M T / V_{5}$ is abnormally susceptible to noise in amblyopia and it is the noise in the stimuli rather than the motion integration deficit per se that causes the impaired performances (Mansouri and Hess, 2006). The second explanation was that an alternative network of neural areas is recruited to support normal plaid perception by the amblyopic eye. Candidate areas included those that have been shown to process with selectivity for coherent plaid motion, i.e., $\mathrm{V}_{1}$ (Guo et al., 2004), V 3 (Gegenfurtner et al., 1997; Wenderoth et al., 1999) and the pulvinar nucleus (Merabet et al., 1998; Villeneuve et al., 2005). To resolve this, Thompson et al., used functional magnetic resonance imaging (fMRI) to compare responses in the visual cortex and thalamus to incoherent and coherent motion of plaid stimuli while they were perceived normally by the amblyopic eye or the non-amblyopic eye (Thompson et al., 2012). Without entering into the details, their results have indicated that while the perception of the plaid stimuli was constant for both amblyopic and non-amblyopic viewing conditions, the network of neural areas that supported this perception was different. Both cortical responses in $\mathrm{MT} / \mathrm{V}_{5}$ and sub-cortical responses in the pulvinar nucleus were correlatively different. To interpret these data, the authors propose that the visual system of strabismic subjects with amblyopia is able to compensate the $\mathrm{MT} / \mathrm{V}_{5}$ deficit by recruiting an alternate network to perceive correctly higher-order motion. The pulvinar nucleus was considered as a very good candidate for that although it might be confirmed in this function.

The principle of convergence is maintained in infantile strabismus, even if the neural bases for perception of respective attributes of the visual scene may be altered. This is reinforced by additional data (in particular from psychophysical measures) reported below.

As a general conclusion:

- The principle of convergence may be extended to all visual cortical areas and the neural networks in which they are included both in normally sighted subjects and those with infantile strabismus. In each case, the elaboration of visual perception is achieved through a coherent link of their activity even some abnormalities exist after strabismus.

- To support our hypothesis, such convergence of information about the different visual attributes both in each superior visual area and within the neural networks in which they are included is also one inescapable condition to consider that the present treatments of infantile strabismus may be possibly improved compared to presently.

\section{PRINCIPLE OF INTERACTIONS AND OF INTER-DEPENDENCY OF ALL THE ATTRIBUTES OF THE VISUAL SCENE}

As developed in section Principle of Convergence in Visual Cortex, the principle of convergence is applicable at any level (neurons, columns, cortical maps and neural networks) in the whole visual cortex both in normally viewing and infantile strabismic subjects, even though the latter have some weaknesses. Indeed, strabismic subjects are still able to quite coherently perceive visual scenes in spite of their visual system abnormalities.

Thus, interactions are facilitated between the various attributes for visual perception whether vision is normal or not. All of the attributes of the visual scene are also inter-dependent. This is fortunate from the perspective we are developing here since interaction and inter-dependency of the various attributes of the visual scene, at any level that they may occur, are also major keys here for considering that rehabilitation of full visual function may be possible after infantile strabismus. This is illustrated below in higher mammals including humans, both during post-natal development and in the adult, through functional and anatomical data obtained by exploring $\mathrm{V}_{1}$ and superior visual cortical areas 
as well as through psychophysical data. Data from mathematical modeling will also be evoked. As usual, we start with subjects with normal view and then discuss subjects with infantile strabismus.

\section{Subjects With Normal View Interactions and Inter-Dependency During Post-natal Development}

First, it is important to emphasize here that the principle of convergence applies to the post-natal period of development. For half a century, we have been known that each neuron in $\mathrm{V}_{1}$ responds to each visual attribute from birth or soon after birth from the day the eyes open (e.g., Hubel and Wiesel, 1963b; Frégnac and Imbert, 1978; Milleret et al., 1988, 1994). A cortical map is also initially present for each attribute of the visual scene (except apparently for direction of movement and high spatial frequencies; see above). The visual responses are initially quite poor and sluggish but they get increasingly stronger and are refined with post-natal visual experience (e.g., Chapman et al., 1996; Crair et al., 1998; Crowley and Katz, 1999; Tani et al., 2012). In parallel, the cortical feed-forward and feed-back networks also develop and refine both anatomically and functionally (e.g., Movshon and Van Sluyters, 1981; Wiesel, 1982; Garey and de Courten, 1983; Innocenti, 1986; Wang et al., 2016). The same holds true for the trans-thalamic cortical routes (Bridge et al., 2016). Interactions and inter-dependency between the different visual attributes thus exist from the earliest stages after birth.

On the basis of the anatomo-functional organization of $\mathrm{V}_{1}$ reviewed above, not surprisingly, a key initial characteristic of $\mathrm{V}_{1}$ neurons is their potential to interact and their interdependency over short and long distances in cortex even when considering only a single attribute of the visual scene. This was investigated for example by Schuett et al. (2001) who studied plasticity of orientation preference maps in $\mathrm{A} 17$ of kittens (i.e., $\mathrm{V}_{1}$ homolog). Pairing a brief visual stimulus of a given orientation $\left(\mathrm{OR}_{1}\right)$ with electrical stimulation of the cortex in a region underlying another orientation $\left(\mathrm{OR}_{2}\right)$, they found that their relative timing determines the direction of plasticity: the recorded neuron changed its orientation preference $\left(\mathrm{OR}_{1}\right)$ toward the orientation of the point that was stimulated $\left(\mathrm{OR}_{2}\right)$ if the cortex was activated first visually and then electrically; by contrast, the cortical response to the paired orientations was diminished if the electrical activation came first. This allowed demonstrating interactions and inter-dependency between columns underlying different orientations within the global orientation map. In addition, they showed that pinwheel centers (singularities in the orientation cortical map; Figure 5B) are less affected by the pairing than the surrounding orientation columns which allowed to conclude that plasticity is however not uniformly distributed within the cortex during the critical period.

Furthermore, the development of the cortical maps corresponding to the different visual attributes may be interdependent. For example, Tani et al. (2012) investigated kitten's primary visual cortex with optical imaging of intrinsic signals and showed that the maturation of the orientation, spatial frequency and retinotopic maps are tightly linked. Among other findings, they indeed demonstrated that orientation maps for lower spatial frequencies appear first in the whole retinotopic map (where the whole visual field is represented) while the orientation maps for high spatial frequencies appear only later in correlation with the maturation of the part of the retinotopic map where the central vision is treated (Tusa et al., 1981). Inter-dependency was also shown by Wang et al. (2010) who reported that orientation preference in $V_{1}$ (of mice) matches binocularly only by the end of the critical period. Through an electrophysiological approach, they indeed observed that the preferred orientation of individual cortical cells is mismatched between the two eyes soon after birth but that the binocular similarity of orientation preference improves and reaches adult levels during the critical period, i.e., with post-natal visual experience (see also Espinosa and Stryker, 2012 for review). This implicitly indicates that orientation preference and ocular dominance are inter-dependent during development.

- Thus, as early as the neural bases of responses to a given attribute develop, this has an impact on the development of the neural bases of other visual attributes. However, the time course of their respective critical periods has to overlap (see above).

\section{Interactions and Inter-Dependency in the Adult Single attributes}

In $\mathrm{V}_{1}$, interactions concerning a single attribute occur first at the level of single neuron, because of the organization of its receptive field. As mentioned in section Convergence at the Level of Single Neurons, each receptive field includes at least a "classical" part as defined by Hubel and Wiesel $(1962,1965)$ evoking spike activity and a larger surrounding "silent" zone sensitive to visual stimulation but without generating any spikes (Bringuier et al., 1999). More recently, three distinct regions have in fact been distinguished (Angelucci et al., 2002; Angelucci and Bressloff, 2006 for review): (i) The "classical" receptive field (or receptive field center); (ii) The "spatial summation" receptive field which also generates spikes during visual stimulation but whose size is contrast dependent when mapped with an optimal size stimulus: smaller when highly contrasted, but larger with low contrast; (iii) The "surrounding" (silent) receptive field which may be inhibitory or excitatory. The latter study also showed that feed-forward retino-geniculo-cortical pathways produce both the "classical" receptive field and the "high-contrast spatial summation" receptive field while intra-cortical feed-forward connections and feed-back connections from higher visual areas produce the "low-contrast spatial summation" receptive field and the "surrounding" (silent) receptive field respectively. These receptive field types in $V_{1}$ are evidence of modulation by contextual stimuli (belonging however to the same attribute family) lying near or far outside the "surrounding" (silent) receptive field. This has been demonstrated for orientations since responses to discrete stimulation within the "classical" receptive field (via light bars or Gabor patches) are often facilitated by iso-oriented stimuli presented in the "high-contrast spatial summation" receptive field while they are usually suppressed when presented in the "surrounding" (silent) receptive field (e.g., Blakemore and Tobin, 1972; Nelson and Frost, 1978; Gilbert and Wiesel, 1990; DeAngelis et al., 1994; Mizobe et al., 2001; Chisum et al., 2003). A specific case of short-range modulation is "colinear facilitation," a phenomenon which is thought to underlie 
perceptual grouping of contour elements (Kapadia et al., 1995; Hess and Field, 1999). The fact that the most superficial feedback projections terminate in layers II-III of $\mathrm{V}_{1}$ in a patchy fashion, showing modular and orientation specificity (see above) is consistent with this proposed role in orientation-specific center-surround interactions. Such latter interactions have also been demonstrated for contrast detection (Levitt and Lund, 1997; Polat et al., 1998; Mizobe et al., 2001). Note that center/surround organization of each receptive field also exists in superior visual areas, for example when encoding of direction of movement and velocity of movement of visual stimuli in MT area is under control sRF (Allman et al., 1985).

Interactions and inter-dependency concerning each single attribute evidently also occur at the level of visual cortical maps. A first example is adaptation-induced plasticity of orientation observed during electrophysiological recordings and intrinsic signal imaging of cortical maps in $\mathrm{V}_{1}$ of adult cats. Following prolonged exposure to a single oriented stimulus, changes of the neuronal responses to orientation occur and the direction of the shifts toward (attractive) or away (repulsive) from the adapter in the orientation global map depends on adaptation duration. This capacity for adaptive changes of the neurons is again not uniformly distributed in visual cortex, i.e., being the highest in iso-orientation domains and the lowest at and near pinwheels. Synchronous firing of orientation selective neurons is also modified during adaptation-induced plasticity (e.g., Dragoi et al., 2000, 2001; Schummers et al., 2005; Ghisovan et al., 2008, 2009; Nemri et al., 2009; Cattan et al., 2014). Experimentally induced synchrony also induces major changes in the orientation maps in adult cats (Godde et al., 2002). Such adaptive processes involve interactions over short and long distances through cortico-cortical connections (Yousef et al., 2001). In summary; adaptation-induced plasticity in the adult visual cortex is a dynamic time-dependent process that involves network interactions and that can lead to response depression and enhancement. A second example is the implication of the feed-back connections from superior visual areas in shaping visual cortical maps in $\mathrm{V}_{1}$, in particular the orientation and direction of movement maps. Suppressing feed-back signals from the posterior parietal cortex in the cat even abolishes the global layout of the direction of movement maps (e.g., Galuske et al., 2002; Tong et al., 2011). With fMRI in humans, Williams et al. (2008) also demonstrated that the foveal zone of $V_{1}$ is sensitive to information about objects presented in the periphery because of feed-back connections from superior visual cortical areas (see also Yu and Shim, 2016).

These data show how interactions in visual cortex allow local signals concerning one attribute to be integrated across visual space to generate global percepts as well as how they contribute to perceptual figure-ground segmentation of visual scenes and contextual modulation (e.g., Gilbert et al., 1996; Seriès et al., 2003).

\section{Interactions and inter-dependency between various visual attributes}

Interactions and inter-dependency between pairs of attributes. There is also evidence that interactions and inter-dependency exist between each pair of visual attributes (see Table 3 and IT section of Table 1). For example, these have been described for spatial frequency and contrast (Enroth-Cugell and Robson, 1966; Campbell and Maffei, 1981), spatial frequency and motion (e.g., Bisti et al., 1985), form (= orientation + spatial frequency) and color perception (e.g., Moutoussis, 2015), ocular dominance and orientation (Crair et al., 1997; Nakagama et al., 2006) as well as for spatial location (retinotopy) and velocity of movement (e.g., Orban et al., 1978).

Interactions and inter-dependency between triplets of visual attributes. Ophthalmologists, orthoptists and optometrists presently concentrate their efforts on the three following visual attributes and their inter-dependency in patients with infantile strabismus: visual acuity of each eye, binocularity and 3D perception, with the binocularity being associated here to the alignment of the eyes through surgery. But interactions and inter-dependency between other triplets of visual attributes have also been described. Thus, for example, as illustrated in Table 4, the trios "orientation, ocular dominance and spatial frequency" (Hübener et al., 1997), "orientation, direction of movement and ocular dominance" (Kim et al., 1999; Buzás et al., 2001) "direction, spatial frequency and contrast" (Lalanne and Lorenceau, 2006) as well as "spatial frequency, temporal frequency (i.e., motion) and color" (Shoham et al., 1997) are also tightly inter-linked. In fact, because of the extensive convergence of visual information within the whole visual cortex, all possible combinations likely lead to interactions.

Interactions and inter-dependency between all of the visual attributes. The question of the interactions and interdependences between all the attributes during visual perception is a key issue here since such interactions participate actively to the elaboration of the global visual perception. Let us examine this at the neuronal level first in $V_{1}$ and then beyond, in superior visual areas.

\section{$\mathrm{V}_{1}$ Area}

We have shown above that $\mathrm{V}_{1}$ contains two distinct populations of neurons: "Pop 1," activated through the $\mathrm{M}$ and/or P channels, which is the largest and has the most convergence of attributes, and "Pop 2," activated through the P and/or K channels, which is smaller and has less convergence (section Convergence at the Level of Single Neurons; Table 1). Pop 1 neurons (simple or complex) have rectangular receptive fields which are activated by orientation, velocity and/or direction of movement and are mostly binocular while Pop 2 includes neurons with circular receptive fields almost exclusively activated by colors through one eye. Correspondingly, we hypothesize that the degree of interactions and inter-dependence between the various visual attributes within each neuronal population corresponds to their degree of convergence. Thus, there would be more attribute interactions in Pop 1 than in Pop 2. But both neuronal populations are activated by the $\mathrm{P}$ channel, which suggests that attributes from each population may also interact and be inter-dependent. The fact that some neurons of Pop 2, located in the blobs in layer II-III, are also sensitive to velocity of movement supports this hypothesis (cf. Table 1). The next paragraph provides further supports for this. 
TABLE 3 | Examples of interactions and inter-dependency of pairs of visual attributes in adults with normal vision.

\begin{tabular}{|c|c|c|}
\hline Interactions & Main results & References \\
\hline RF size/Contrast & The static RF features vary with contrast and contrast adaptation in $V_{1}$ of macaque. & (Durand et al., 2012) (P) \\
\hline OR/Contrast & $\begin{array}{l}\text { - See section Single Attributes } \\
\text { - Neurons in primate } V_{1} \text { and } V_{2} \text { responding selectively to orientated luminance contours and } \\
\text { neurons signaling non-luminance defined contours are distributed invariably in all the OR } \\
\text { columns }\end{array}$ & $\begin{array}{l}\text { (Angelucci and Bressloff, 2006) (M) } \\
\text { (An et al., 2014) (OI) }\end{array}$ \\
\hline SF/Contrast & The sensibility to contrast (1/C) varies with SF with an optimal at a certain SF & $\begin{array}{l}\text { (Enroth-Cugell and Robson, 1966) (P); } \\
\text { (Campbell and Maffei, 1981) (PSY) }\end{array}$ \\
\hline OR/RET & Alternative view: both $\mathrm{OR}$ and RET maps in $\mathrm{V}_{1}$ are independent & $\begin{array}{l}\text { (Buzás et al., 2003) (OI) } \\
\text { (Paik and Ringach, 2012) (M) }\end{array}$ \\
\hline OR/Movement (TF) & $\begin{array}{l}\text { - An apparent speed (perceptive illusion depending on the context) is OR dependent } \\
\text { - Shape and motion interact at perceptual and attentional levels during processing of structure } \\
\text { - } \text { - Brom motion stimuli } \\
\text { segmentivity is higher during perceptual form/motion integration (binding) than during } \\
\text { - Perceptual alterations between unbound moving contours and bound shape motion engage } \\
\text { a ventral/dorsal interplay } \\
\text { - Local form-motion interactions influence global form perception } \\
\text { - } \beta \text { but not } \gamma \text { band oscillations index visual form-motion integration } \\
\text { - Local (OR) and global (MV) information are already linked in } V_{1}\end{array}$ & $\begin{array}{l}\text { (Seriès et al., 2002) (PSY) } \\
\text { (Georges et al., 2002) (PSY) } \\
\text { (Miskiewicz et al., 2008) (PSY) } \\
\text { (Aissani et al., 2011) (MEG-PSY) } \\
\text { (Caclin et al., 2012) (PSY) } \\
\text { (McCarthy et al., 2012) (PSY) } \\
\text { (Aissani et al., 2014) (PSY) } \\
\text { (Gérard-Mercier et al., 2016) (P) }\end{array}$ \\
\hline Natural scene/MV & Optimal speed estimation in natural image movies predicts human performance & (Burge and Geisler, 2015) (PSY) \\
\hline SF/MV & SF in $V_{1}$ depends on the motion velocity (MV) of the visual stimulus & (Bisti et al., 1985) (P) \\
\hline Motion/Color & $\begin{array}{l}\text { - Color and motion are linked during visual perception } \\
\text { - But whether the binding between both occurs as early as } V_{1} \text { is still under debate }\end{array}$ & $\begin{array}{l}\text { (Blaser et al., 2005) (PSY) } \\
\text { (Linares and López-Moliner, 2006) (PSY) } \\
\text { (Zhang et al., 2016) (PSY) }\end{array}$ \\
\hline RET/Motion & $\begin{array}{l}\text { Velocities encoded in visual cortex increase with eccentricity within the visual field (i.e., larger in } \\
\text { periphery than centrally) }\end{array}$ & (Orban et al., 1978) (P) \\
\hline
\end{tabular}

RET, OR, SF, MV, DIR and OD, same as in Table 1. RF, receptive field; PW, pinwheel; TF, temporal frequency. P, physiology; Ol, optical imaging; 2P, two-photon microscopic imagery; MEG, magneto-encephalography; ANAT, anatomical study; PSY, psychophysics; M, models.

Figure 5C shows that, viewed from the surface, $V_{1}$ appears as a retinotopic map of visual space with "overlapping" functional spatially periodic maps that represent stimulus features such as edge orientation, direction of movement, spatial frequency etc. But, during visual perception, all these maps have to interact in a coordinated way. Their spatial relationships may ensure that all combinations of stimulus features are represented uniformly across the visual field. But the question here is "How?" Both experimental approaches and theoretical models attacked this question. From these studies, two main principles have emerged. First, cortical maps in V1 are optimized for uniform coverage. This principle permits continuous smooth mapping of stimulus properties across the cortical surface, and "coverage uniformity" that is uniform representation of combinations of map features over visual space (Swindale, 1991, 2000; Swindale et al., 2000). Here stimulus features such as edge orientation, velocity/direction of movement and spatial frequency are thought to be encoded in $V_{1}$ by overlapping feature maps arranged so that the location of neurons activated by a particular combination of stimulus features can be predicted 
TABLE 4 | Illustrating interactions (or frames of interactions) occurring between 3 and 4 visual attributes in normally viewing adult: RET, OR, PW, SF, MV, DIR and OD; P, OI, 2P, ANAT, PSY, M, same abbreviations as in the other Tables.

\begin{tabular}{|c|c|c|}
\hline Interactions & Main results & References \\
\hline OR/OD/Color & $\begin{array}{l}\text { - } \mathrm{OR} \text { and } \mathrm{OD} \text { maps in } \mathrm{V}_{1} \text { cross systematically approximately at right angle } \\
\text { - Most } \mathrm{PW} \text { in } \mathrm{OR} \text { maps and about } 50 \% \text { of the blobs in } \mathrm{V}_{1} \text { coincide with the center of OD domains } \\
\text { - The OR and color maps in } \mathrm{V}_{1} \text { are each only loosely related to OD maps }\end{array}$ & $\begin{array}{l}\text { (Bartfeld and Grinvald, 1992) (OI } \\
+ \text { ANAT) } \\
\text { (Landisman and Ts'o, 2002) (OI } \\
+ \text { P) }\end{array}$ \\
\hline OR/Color/Luminance & OR perception is influenced by both chromatic and luminance & (Clifford et al., 2003) (PSY) \\
\hline OR/OD/SF & $\begin{array}{l}\text { - } \mathrm{OR} \text { and } \mathrm{OD} \text { maps in } \mathrm{V}_{1} \text { often cross at right angles while most } \mathrm{PWs} \text { are concentrated in the center } \\
\text { regions of the } \mathrm{OD} \text { columns } \\
\text { - albeit weaker than for OR/OD, geometric relationships are also observed between the OR and SF } \\
\text { domains. } \\
\text { - The OD and SF maps in } \mathrm{V}_{1} \text { are also spatially related: there is a tendency for the low SF domains } \\
\text { to avoid the border regions of the OD columns. }\end{array}$ & (Hübener et al., 1997) (OI) \\
\hline ON-OFF/RET/OR & $\begin{array}{l}\text { The spatially separate ON and OFF subfields of simple cells in layer II-III of } V_{1} \text { exhibit topographically } \\
\text { distinct relationships with RET and OR maps }\end{array}$ & (Lee et al., 2016) (2P) \\
\hline OR/DIR/OD & $\begin{array}{l}\text { - OR, DIR, and OD maps in } V_{1} \text { are represented in the cortex in a mutually dependent manner } \\
\text { - Baskets cells in } V_{1} \text { establish connections with OR, DIR, and OD maps }\end{array}$ & $\begin{array}{l}\text { (Kim et al., 1999) (OI) } \\
\text { (Buzás et al., 2001) (OI + ANAT) }\end{array}$ \\
\hline SF/TF/Color & $\begin{array}{l}\text { Cytochrome oxidase within blobs of } V_{1} \text { coincide with domains engaged in the processing of low SF } \\
\text { and high TF contents of the visual scene }\end{array}$ & (Shoham et al., 1997) (OI) \\
\hline RET/OR/Color & $\begin{array}{l}\text { Apparent color-orientation bindings in the periphery can be influenced by feature binding in central } \\
\text { vision }\end{array}$ & (Suzuki et al., 2013) (PSY) \\
\hline OR/RET/SF & $\begin{array}{l}\text { High SFs and low SFs activate OR maps in } V_{1} \text { when central and peripheral vision are respectively } \\
\text { concerned }\end{array}$ & (Ribot et al., 2013) (OI + M) \\
\hline
\end{tabular}

$R F$, receptive field; $T F$, temporal frequency. MEG, magneto-encephalography.

from the intersections of these maps. An alternative principle has been proposed stipulating that patterns of activity elicited by complex stimuli are best understood in the framework of a single map of spatio-temporal energy rather than by overlapping and intersecting multiple maps. The authors supporting such principle (e.g., Basole et al., 2003, 2006; Mante and Carandini, 2005) indeed observed that a single population of neurons can be effectively activated by multiple combinations of orientation, length, motion axis and speed.

\section{Superior Visual Cortical Areas}

Considering the convergence of the three channels $\mathrm{M}, \mathrm{P}$, and $\mathrm{K}$ within both the dorsal and the ventral streams and the respective functional roles of each stream in visual processing, it is not surprising that multiple interactions occur between all the visual attributes within both streams (see Table 2 for details; cf. also section Evaluation of Convergence in Each Superior Visual Cortical Area).

Both streams are also linked through numerous reciprocal and unidirectional connections (e.g., Ungerleider et al., 2008; Pollen, 2011; see also Cloutman, 2013 for review). This enables additional interactions and inter-dependences. Functional data indeed support this notion. For example, such interactions may allow "integrating" simultaneously faces, houses, motion and action (Keizer et al., 2008). Such cooperation allows the perception of 3D object shape from $2 \mathrm{D}$ random-dot motion (Iwaki et al., 2013). It has also been demonstrated that a circumscribed damage to ventral stream impairs central motion perception, even for non-form motion (Gilaie-Dotan et al., 2013); cf. Table 5 for some more examples.

- Thus, all attributes of the visual scene interact and are interdependent during the elaboration of visual perception. This occurs in $\mathrm{V}_{1}$ and is further processed at the level of the superior visual cortical areas.

\section{Strabismic Subjects Before Any Medical Intervention}

Considering that the visual system sustains its general organization in spite of abnormal vision, the attributes of the visual scene also interact after infantile strabismus and are thus inter-dependent during the elaboration of visual perception. But, as a general rule, when brain processing and hence perception of one given visual attribute is altered, this has a negative impact on others. Such alteration might be even more extended than might be expected. Thus, for example, infantile strabismic amblyopes show localization deficits that are larger than expected when considering their losses in spatial resolution (Kiorpes et al., 1993). Also, thresholds for depth perception are increased when acuity is decreased (Husk et al., 2012); cf. Table 6 for more examples. Accordingly, the two general principles of interaction and inter-dependence also apply to cortical maps of subjects with infantile strabismus. Farley et al. (2007) even proposed that the global visual alteration resulting from infantile strabismus results in a "coordinated reorganization of multiple visual cortical maps." 
TABLE 5 | Interactions between the dorsal and ventral streams, implicating interactions between all the attributes of the visual scene.

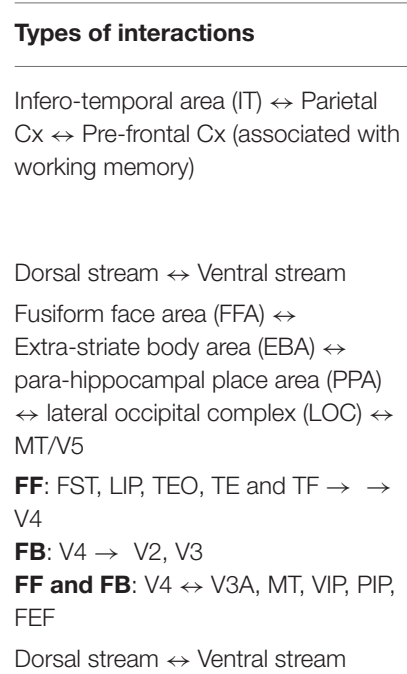

(Goodale et al., 2005) - Review
(Spiridon et al., 2006) (fMRl)

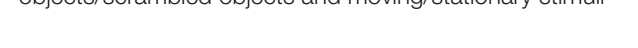

Humans simultaneously integrate images of faces, houses, motion and action. This is only possible through a binding process, with interactions between both the ventral and dorsal streams.

The $V_{1} N_{2}$ complex and ventral cortical areas $V_{3}, V_{4}$ together with dorsal cortical areas LIP, VIP and 7a with additional contributions from motion areas MTN5, FST and MST together with their sub-cortical relations have the physiological properties required to constitute a "posterior perceptual core" that underlies the normal primary perceptual experience of image content, space and sense of minimal self.

The perception of 3D object shape from 2D random-dot motion implicates cooperation between both the dorsal and ventral streams.

Circumscribed damage to ventral visual cortex impairs central motion perception, even for non-form motion

Information is transferred directly between the ventral and dorsal streams at multiple stages and locations along their trajectories

Color perception through S-cones implicates both the ventral and the dorsal streams. The combination of both signals would facilitate the extraction of shape-from-shadow signals to benefit global scene analysis and motion perception

Dorsal and ventral attention systems use distinct circuits but they display collaborative roles Posterior parietal cortex drives infero-temporal activations during 3D object vision
(Keizer et al., 2008) (PSY)

(Pollen, 2011) - Review

\author{
(Iwaki et al., 2013) (PSY) \\ (Gilaie-Dotan et al., 2013) (PSY) \\ (Cloutman, 2013)-Review \\ (Conway, 2014) - Review
}

(Vossel et al., 2014) -Review

(Van Dromme et al., 2016) (fMRI)

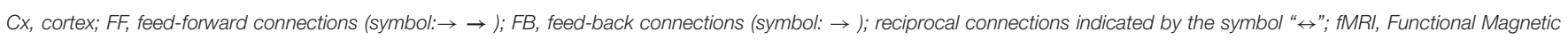
Resonance Imaging. Other abbreviations, same as in other Tables.

- The entirety of visual cortex follows both the principle of convergence and the principle of interactions and of interdependencies between the different attributes of the visual scene both in normally viewing and infantile strabismic subjects.

- Such principles may thus also apply in case of visual rehabilitation after infantile strabismus.

The latter is a major point in the present context since our main hypothesis here is that if improving the perception of at least one given attribute of the visual scene after early strabismus, after and even before eye surgery, it will improve the perception of some (if not all) other attributes. This hypothesis leads to interesting consequences, for example, that improving acuity through therapy will also be beneficial for spatial localization as well as the perception of movement etc... A few examples supporting this hypothesis will be evoked in section Data Which Support Our New Perspective.

\section{IMPACT OF THE VISUAL SYSTEM ON OTHER SENSORY AND MOTOR SYSTEMS}

Many sensory and motor systems in higher mammals are strongly vision-dependent and are thus also affected in case of infantile strabismus. They are also of main interest here since rehabilitation of vision might also restore these systems and their associated functions. In other words, rehabilitation after infantile strabismus likely might be extended far beyond visual perception.

A good model to approach this issue is postural stability, which plays a major role for equilibrium and orientation of the body (e.g., Rougier and Lacour, 2006 for review). Of course, this very complex process requires various sensorymotor interactions, and depends on various skeletal segments and muscles whose actions are controlled by static and dynamic reflexes and dependent on attention. But postural stability is also strongly under the influence of visual, somato-sensory (including proprioception) and vestibular sensory systems as well 
TABLE 6 | Examples illustrating interactions between at least 2 visual attributes in adult mammals with infantile strabismus.

\begin{tabular}{|c|c|c|}
\hline Interactions & Main results & References \\
\hline $\begin{array}{l}\text { Spatial } \\
\text { location/acuity }\end{array}$ & $\begin{array}{l}\text { Infantile strabismic amblyopes show localization deficits that are large relative to their losses in spatial } \\
\text { resolution (in contrast with anisometropic amblyopes) }\end{array}$ & (Kiorpes et al., 1993) (PSY) \\
\hline $\begin{array}{l}\text { Contrast } \\
\text { sensitivity/ } \\
\text { vernier acuity }\end{array}$ & $\begin{array}{l}\text { When Vernier stimuli are equal in terms of effective contrast, the extent of the Vernier acuity deficit is } \\
\text { reduced to approximately the extent of the spatial resolution deficit }\end{array}$ & (Kiorpes et al., 1993) (PSY) \\
\hline OR / OD & $\begin{array}{l}\text { In contrast to normally raised animals (cats), PW centers (in OR maps) after infantile strabismus no longer } \\
\text { show consistent topographical relationship to the peaks of OD domains although OR and OD maps } \\
\text { continue to cross orthogonally }\end{array}$ & (Engelmann et al., 2002) (OI) \\
\hline SF/OR & After infantile strabismus, the OR map in $V_{1}$ is altered with weakest (amblyopic) eye viewing only low SF & (Schmidt et al., 2004) (OI) \\
\hline $\begin{array}{l}\text { Acuity/ } \\
\text { depth perception }\end{array}$ & Depth thresholds are increased while acuity is decreased & (Husk et al., 2012) (PSY) \\
\hline
\end{tabular}

OR, PW, SF, OD, OI, PSY, abbreviations same as in other Tables.

as oculomotricity (e.g., Nashner, 1976; Horak, 2010; see also Paillard, 1971, 1976; Massion, 1994, 1997 for reviews).

The impact of vision on postural stability is rather well documented. The simplest evidence is that postural stability is better when both eyes are open than when they are closed (e.g., Baron, 1950; Gagey et al., 1973; Paulus et al., 1984; Isotalo et al., 2004; see also for example Guerraz and Bronstein, 2008 for review). Not surprisingly, blindness has dramatic consequences on posture (e.g., Alotaibi et al., 2016). This vision-dependence of posture has also been demonstrated in infantile strabismic subjects. As indicated in the Introduction, infantile strabismus also impairs postural stability (Marucchi, 1987; Marucchi and Gagey, 1987; Lions et al., 2014; Ezane et al., 2015). But of particular interest here the quality of vision in case of infantile strabismus (even before any surgery) has a substantial impact onto postural stability. Thus, Matsuo et al. (2006) reported that infantile strabismic children with some (even poor) binocular vision display better postural performance than those with none. Also, Gaertner et al. (2013) showed that bi-ocular visual stimulation improves control of posture for both near and far distances, in cases of convergent and divergent strabismus. Finally, even though vision is altered because of infantile strabismus, the postural parameters are also significantly better with the eyes open than when they are closed (Legrand et al., 2011).

The impact of the somato-sensory system through extraocular proprioception on postural stability is also well established in the literature. Recall that extraocular proprioceptive inputs originate from receptors within the extraocular muscles which are mainly located in the tendons and which project through the ophthalmic branch of the Vth nerve to multiple sites in the CNS (e.g., Milleret, 1988; Buisseret, 1995; Donaldson, 2000 for reviews). These receptors provide information about the position of the eyes to the brain during eye movements, including for example at the level of the vestibular nuclei (Ashton et al., 1988, 1989). Thus, under normal conditions, the extraocular proprioception contributes to maintain body equilibrium (Ushio et al., 1975, 1980; Eber et al., 1984; Roll et al., 1989). In infantile strabismus, the general idea is that such extraocular proprioception is unbalanced because of the misalignment of the eyes, thus contributing to generate postural instability (e.g., Legrand et al., 2011; Ezane et al., 2015; Bucci et al., 2016; Lions et al., 2016).

- We propose here that any improvement of vision and/or extraocular proprioception after eye surgery (or any other appropriate therapeutic protocols) might also lead to functional improvement of vision-dependent systems including postural stability. Reshaping and re-equilibration of various neural networks would be required for this but brain plasticity is likely able to solve this problem whatever the age.

\section{DATA WHICH SUPPORT OUR NEW PERSPECTIVE}

The three principles of convergence, interaction and interdependency underlie the processing of the various attributes in visual cortex during the elaboration of visual perception. Furthermore, the visual system influences other systems such as sensory and motor systems (cf. section Impact of the Visual System on Other Sensory and Motor Systems). Based on these "rules" our hypothesis here is that the rehabilitation of perception after infantile strabismus should be substantially extended compared to presently. In fact, as developed below, this is already supported by some data in literature established by fundamental research.

\section{Rehabilitation of Visual Perception Present Actions of Ophthalmologists and Collaborators in Cases of Infantile Strabismus}

In the clinic, the different forms of infantile strabismus are observed to affect both the sensory and the motor aspects of visual perception. It is also well known that infantile strabismus affects other abilities like postural stability (cf. section Impact of the Visual System on Other Sensory and Motor Systems). It has also been shown (in 55 children from 7 to 15 years old) that nonverbal abilities are altered in cases of infantile strabismus, with a significant relation between infantile strabismus and constructive praxia $(p=0.009)$, visual memory $(p=0.037)$, strategy formation (0.040) and the quality of drawings (Gligorović et al., 2011). Whether this is a direct consequence of infantile strabismus 
or whether the abnormal cerebral patterns (abnormal neuronal activities, asynchrony) which lead to infantile strabismus are the primum movens of both infantile strabismus AND other disabilities is presently unknown however.

Regardless of the answer, recall that ophthalmologists, orthoptists and optometrists presently focus the treatment of infantile strabismus on only three major items:

1) Visual acuity. The monocular strabismic amblyopia must be avoided and cured in all cases. This is performed classically through patching or through optical or pharmacological penalization of the fellow (non-deviated) eye to increase vision of the amblyopic (deviated) eye. The patching treatment is effective and sufficient in most cases. However, sometimes the effectiveness of these procedures may be limited because of poor compliance and variable outcomes. Additionally, if the amblyopia is severe, these treatments are difficult to initiate; amblyopia treatment can also be less effective if initiated after age 10. This partially explains the reason why additional "new" techniques such as "binocular therapy" have been proposed (cf. section Rehabilitation of Monocular Vision in Each Eye).

2) Alignment of the eyes. Eye alignment is adjusted through optical treatment and/or surgery. In some forms of infantile strabismus such as pure accommodative strabismus (which is a late-onset strabismus), only an optical treatment of hyperopia allows alignment of the eyes.

3) Rehabilitation of binocular vision. Rehabilitation can only be achieved in patients who do not display an early-onset infantile strabismus (i.e., developed before 12-24 post-natal months), and in patients for whom monocular amblyopia is avoided or cured AND alignment of the eyes is obtained.

Even focusing on these particular items, several challenges remain in the management of infantile strabismus. In addition to those evoked in the Introduction, two others exist. First, both amblyopia and infantile strabismus are linked since infantile strabismus may cause amblyopia, and also an amblyopic eye may become strabismic. However, so far, new therapeutic tools of treatment for visual rehabilitation mainly focus on the treatment of amblyopia in general and not on strabismic amblyopia only. Also, whether rehabilitation could be addressed to infantile strabismus without amblyopia remains unclear. This interdependency between amblyopia and strabismus along with the lack of effective new therapeutical tools are obstacles to reach a global rehabilitation of all visual and extra-visual aspects of infantile strabismus. Second, the mechanisms which lead to the development of amblyopia (including strabismic amblyopia) are presently rather well known, although still discussed (cf. for example Hess et al., 2009; Clavagnier et al., 2015). Due to these mechanisms, which implicate malleable neural networks, amblyopia may also be treated relatively easily through peripheral treatments (see above for details). In contrast, the mechanisms responsible for infantile strabismus and the lack of binocular vision remain less precisely identified. Most infantile strabismus at least seems however to result mostly from central and robust abnormalities which develop pre- or post-natally (Barnes et al., 2001; Bui Quoc and Milleret, 2014). It is therefore the cortical deficits which must be addressed. The same may apply to subjects with late infantile strabismus whose lack of binocular vision cannot be managed. But ophthalmologists, orthoptists and optometrists are still wondering how to treat the brain of infantile strabismic patients (Tychsen, 2005). Presently all treatments are systematically peripheral and, not surprisingly, are not always successful.

On the other hand, the rehabilitation of the other altered aspects of visual perception, concerning spatial location, contrast sensitivity, motion or orientation detection and color vision has not been considered to date and therefore must be developed. In particular, the abnormal perception of the deviated eye (even after realignment) must be restored (Economides et al., 2012). This also applies to the likely abnormal perception deriving from the non-deviated eye (see above). However, medical and paramedical professionals currently lack tools to both evaluate and rehabilitate these aspects of visual perception. Hopefully, the hypothesis developed here will inspire improvements for this.

\section{Data Showing That Rehabilitation of Vision May Be Improved After Infantile Strabismus}

Some data in the literature already support the hypothesis we are developing here. Of particular interest, they have been obtained by using only non-invasive innovative techniques.

\section{Rehabilitation of monocular vision in each eye}

As underlined above, preventing and/or treating amblyopia of the deviated eye is the first condition to rehabilitate vision in the case of infantile strabismus. Monocular equilibrium and maximal vision in both eyes are absolutely necessary to then have good chances to obtain normal binocular vision (whether or not eye surgery is involved). Thus current efficient techniques to penalize the good eye forcing the amblyopic eye to improve are imperative. On the basis of the present knowledge, the efficiency of this technique is acknowledged, even if each eye works separately. Indeed, for a short period of time, penalization/patching may be permanent until obtaining iso-acuity. But it may then be conducted part-time, which does NOT constantly make each eye work separately.

However, it may be of interest to complement the patching method by a binocular therapy to eliminate amblyopia and promote cooperation between the two eyes. R. Hess, B. Thompson and their collaborators are developing precisely such an approach and are among the leaders in the field (e.g., Baker et al., 2007; Mansouri et al., 2008; Hess et al., 2010a,b, 2011; To et al., 2011; Black et al., 2012; Zhou et al., 2012; Li et al., 2013, 2015; see also Hess and Thompson, 2013, 2015; Hess et al., 2014a,b for reviews). In brief, the method is based on a dichoptic stimulation which aims to increase acuity and decrease the interocular suppression phenomenon in amblyopia. Of particular interest here, to reach equal stimulation, the contrast of the image presented to the amblyopic eye is increased while the contrast of the image presented to the fellow fixing eye is decreased. We must note however that nowadays this technique has mainly proven effective in adults because testing on children is much more difficult to access. Whether it is applicable to all types of strabismus also remains unclear. Presently at least, binocular 
therapy thus cannot be considered as an alternate method to replace patching or penalization.

Also of interest, quite recently, Marc Bear and his colleagues have proposed a novel and promising technique to restore acuity which is completely different from the above described ones. They indeed hypothesized that "a period of retinal inactivity can reduce the threshold for synaptic potentiation such that subsequent visual experience promotes synaptic strengthening and increased responsiveness in the visual cortex" (Fong et al., 2016). To test this hypothesis, they used two different animal models. In young mice, they have shown that the otherwise stable loss of cortical responsiveness caused by monocular deprivation is reversed when binocular visual experience follows temporary inactivation of the retinas (through binocular intravitreal tetrodotoxin [TTX] injection). In 3 month-old kittens, they have shown that a severe impairment of visual acuity is also fully reversed by binocular experience following treatment (again binocular intra-vitreal TTX injection) and, further, that prolonged retinal inactivation alone can erase anatomical consequences of monocular deprivation. They conclude that temporary retinal inactivation represents a highly efficacious means to promote recovery of function. It indeed reveals to be able to "reboot" the brain, which allows the lazy (amblyopic) eye to recover acuity permanently, without any detriment to the strongest (fixing) eye. Until now, such a technique has only been applied to animals with amblyopia. The authors now plan to determine whether the treatment might also be suitable for clinical use in humans.

- Although not included yet in the current treatments of amblyopia after infantile strabismus by medical and paramedical professionals, at least the data obtained by Hess and his group obtained through the "binocular therapy" strengthen the hypothesis we have developed here since modifying the contrast of patterns presented to each eye may have a positive impact on the perception of the high spatial frequencies, i.e., acuity.

\section{Rehabilitation of binocular vision and $3 D$ perception}

Binocular vision is also abnormal in the case of infantile strabismus and/or amblyopia (strabismic amblyopia). Several protocols have been developed to restore it and some are also based on the principles developed above (section Principle of Convergence in Visual Cortex and Principle of Interactions and of Inter-Dependency of all the Attributes of the Visual Scene).

As previously discussed, balanced monocular vision in both eyes is necessary for restoring binocular vision, in combination with the alignment of the eyes. Dichoptic stimulation has been used in the twentieth century for the treatment of infantile strabismus and amblyopia, and orthoptic treatment of abnormal retinal correspondence has attempted to reestablish binocularity. Unfortunately, this has been proven ineffective with the additional risk of creating a permanent diplopia. For these reasons, although interesting, innovative and promising, dichoptic vision training such as that proposed by R. Hess and B. Thompson' work must be considered with caution even if such training rehabilitates $3 \mathrm{D}$ perception in some cases.
Brain stimulation is another option. It aims at both readjusting the "excitatory/inhibitory balance" and re-equilibrating the "cortical synchronization" (Deco et al., 2014) which are both altered in infantile strabismus (cf. above). Of interest here, transcranial direct current stimulation of the brain has also been recently shown to target specific processing channels in human visual cortex (Costa et al., 2015). This method is already currently used in clinics in cases of brain disorders such as epilepsy, bipolarity, Parkinson's disease and schizophrenia (e.g., Koch, 2013; Kimiskidis et al., 2014; for reviews). On such bases, Benjamin Thompson, Robert Hess and their collaborators have compared the effect of dichoptic treatment alone and in combination with visual cortex trans-cranial direct current stimulation on measurements of binocular (stereopsis) and monocular (visual acuity) visual function in a group of 16 young adults (mean age $22.1 \pm 1.1$ years) with amblyopia. It was shown that the dual treatment leads to greater improvements in stereoacuity than dichoptic treatment alone (e.g., Thompson et al., 2010; Clavagnier et al., 2013; Spiegel et al., 2013b).

Another approach is the use of motion stimulation which can enhance stereopsis. In 120 normal children and 30 strabismic patients (9 esotropia, 14 exotropia, 7 intermittent exotropia), Handa et al. (2010) studied the binocular performance with static or moving stereograms. In exotropic patients, they found that 19 subjects (90.4\%) succeeded in performing the moving stereogram test whereas $13(61.9 \%)$ succeeded in only performing the static stereogram test, suggesting a possible effect of motion stimulation in improving stereopsis.

- Equalizing acuity (or almost so) in both eyes increases stereoacuity and thus binocular vision. The use of moving stimuli also improves stereopsis and therefore binocularity. These examples again support our hypothesis.

\section{Rehabilitation of contrast sensitivity}

Contrast sensitivity may also be improved through dichoptic training in case of amblyopia. Quite recently, Li et al. (2015) demonstrated this in 30 adults after 10 days of dichoptic training delivered through a dichoptic video game viewed through video goggles $(n=15)$ or an iPod touch equipped with a lenticular overlay screen $(n=15)$. It should be noted that similar improvement has been observed with the two methods for all spatial frequencies which were tested.

Comparing contrast sensitivity before and after anodal transcranial direct current stimulation in 8 of 13 amblyopic adults, Spiegel and colleagues showed that contrast sensitivity may also be transiently improved using such an approach (Spiegel et al., 2013a,b). In the same study, the authors also demonstrated that the activation of the visual cortex itself may be normalized in individuals with amblyopia after this type of stimulation.

Finally, video game training itself (without any specific dichoptic device) has also been shown to enhance contrast sensitivity in case of amblyopia (Li et al., 2009).

- Consistent with our principles of interaction and of interdependency, these results demonstrate that at least acuity and contrast sensitivity are tightly inter-linked: increased acuity may increase contrast sensitivity and vice versa. 
- The data presented above also show that these types of interactions apply to a large range of spatial frequencies, which is, of course, beneficial for visual perception in the present context.

- Increasing synchrony within the visual cortex, by functionally influencing the neuronal networks underlying the treatment of information in relation to all the visual attributes, may contribute to the rehabilitation of contrast sensitivity.

Note that the data summarized above have been obtained from a wide range of ages: children from $\sim 5$ years and adults including elderly subjects, with however a predominance of adults. This allows underlying the existence of brain plasticity late in life (e.g., Mansouri et al., 2014) which likely results from the modulation of the inhibitory intra-cortical networks (Milleret and Buser, 1984, 1993; Watroba et al., 2001; Baroncelli et al., 2011). Once again, this is fundamental in the present context of rehabilitation since our final goal is to extend rehabilitation of vision after infantile strabismus as much as possible, i.e., in terms of both perception and age.

\section{Motion detection and orientation assessment}

Amblyopic subjects, whether strabismic or not, have also been shown to exhibit a global deficit in motion detection in both eyes. Thus, for example, Davis et al. (2008) reported that the peaks of motion-onset visually evoked potentials (VEPs) observed after visual stimulation of the amblyopic (strabismic) eye occur later than the ones observed from the fellow (non-strabismic) eye in visual cortex of adult patients with early- or late-onset strabismic amblyopia. These same authors also found that the peak times of VEPs observed after visual stimulation of the amblyopic and the fellow eyes are shorter than normal. Accordingly, Thompson et al. (2008) reported that the perception of moving patterns is abnormal in both eyes. Abnormal cortical processing of pattern motion in areas MT and MST of the dorsal stream has also been shown with functional magnetic resonance imaging (Thompson et al., 2012). Moreover, using a psychophysical approach, Husk et al. (2012) showed that structure-from-motion processing, which requires cooperation between both the dorsal and ventral streams (see Table 2) is altered. The efficiency in processing local orientation (implicating the ventral stream, where orientation coherence is processed) has also been reported to be poorer than normal (Husk and Hess, 2013). But as far as we are aware, no study has yet proposed any new therapeutic techniques that would focus on the improvement of these altered components in cases of infantile strabismus or amblyopia.

\section{Color vision}

In patients with late- but not early-onset strabismic amblyopia, Davis et al. (2008) have also reported that the peak times for color VEPs are significantly longer than normal when the amblyopic eye is stimulated. However, as with motion and orientation detection, we are not aware of any study that has ever examined any possible improvements in color vision after treatment of infantile strabismus. Not any improvement of strabismus using rehabilitation techniques focusing on color vision has been reported either.

\section{Rehabilitation of Posture After Strabismus Surgery Through Visual Perception}

In section Impact of the Visual System on Other Sensory and Motor Systems, we discussed how the quality of vision of infantile strabismic children positively influences postural stability before any medical intervention, even if this vision is poorer than normal. We also discussed that oculomotricity contributes actively to posture at least through extraocular proprioception.

In addition, it has been established recently that: (a) postural control of children with infantile strabismus may be improved progressively within the weeks (2-8) following eye surgery (Legrand et al., 2011); (b) wearing prisms instead of having eye surgery alters rather than improves postural stability (Legrand et al., 2012). For these authors, the new "relations" that exist between the extraocular muscles and the CNS are thus of vital importance in the process of improving posture after eye surgery; (c) the quality of visual inputs, in terms of acuity and binocular vision at least, is of great importance in improving postural stability after infantile strabismus surgery (Lions et al., 2016); (d) The improvement of extraocular proprioceptive information which results from eye surgery also plays a major role in improving postural control (Bucci et al., 2016). The latter observation is directly linked to the improvement of the quality of eye movements after eye surgery. It has been demonstrated that the speed and accuracy of saccades, the vergence and the combined eye movements (and therefore binocular coordination) are generally poor before eye surgery and much better after eye surgery (Bucci et al., 2002, 2009).

- From this example, in agreement with our hypothesis, we demonstrate that rehabilitation after surgery for infantile strabismus may also take place in domains other than acuity, ocular alignment and binocularity (thus stereopsis), and even far beyond the visual system itself. Postural stability indeed involves very complex sensory-motor networks extending from the head to the feet and vice versa.

- Some questions however remain such as: (i) Does the rehabilitation of postural components by strabismus eye surgery leads to rehabilitation to normal performance levels? (ii) If yes, how long would such rehabilitation take? (iii) Are performance levels sustained over time? (iv) How much postural rehabilitation may be expected after treatment of different types of strabismus? etc... These questions are presently under study.

- With more knowledge and by installing adequate infrastructure (for both specific medical follow-up and training), our view is that, in the near future, it might become possible to optimize the rehabilitation of many children with infantile strabismus, far beyond visual perception, including postural stability. This could occur after eye surgery but we suggest that such improvements could be achieved by combining surgery with other methods, including daily visual experience and perhaps specific visual trainings (see below). We also believe that such rehabilitation might also be extended to adult subjects. 


\section{CONCLUSION}

In cases of infantile strabismus (for recall occurring during childhood), with either early-onset or late-onset, ophthalmologists, orthoptists and optometrists presently aim at rehabilitating vision by focusing on monocular visual acuity (prevention and treatment of amblyopia), motor balance/symmetry/alignment of the eyes, extraocular proprioceptive balance/symmetry in both eyes and binocularity/stereopsis. The perceptual alterations of the other attributes of the visual scene such as spatial location, orientation, velocity/direction of movement, contrast and color are therefore not treated. Yet such attributes play a major role during visual perception. The alterations of vision-dependent systems which, for example, lead to the alteration of postural stability, are also not explicitly taken into account. This would be useful though since, among other functions, postural stability affects locomotion and orientation of body in space. Medical and paramedical practitioners are aware of this problem. However, as outlined above, appropriate tools do not currently exist to address these issues. In addition, the incomplete knowledge of the complex relationships between strabismus and the brain is an impediment. Here, to deal with this problem, we propose that rehabilitation of perception after infantile strabismus may be extended from present practices, possibly with relative ease and/or by using non-invasive approaches.

Based on the present knowledge of the organization of the visual system in higher mammals including humans, our first proposal here is that the current visual rehabilitation might be extended to ALL (or almost all) of the attributes of the visual scene which are altered in case of infantile strabismus. The information related to all these attributes converge, interact and are inter-dependent with numerous common targets within the visual system, in particular at the level of the visual cortex, from $\mathrm{V}_{1}$ and beyond, from individual neurons to complex neuronal visual networks. If the perception of one given visual attribute is improved, for example high spatial frequencies (i.e., acuity), we would expect that this might also improve the perception of other (if not all) visual attributes. For instance, this would mean that the practice of addressing only high spatial frequencies, as is currently the case in the treatment of monocular amblyopia, could in itself improve the perception of the other visual attributes in cases of amblyopia and/or strabismus. Two neuronal populations are however distinguishable in $\mathrm{V}_{1}$, namely the populations Pop 1 and Pop 2, which differ both by their size and their amount of convergence (cf. section Convergence at the Level of Single Neurons and Table 1). Since the population Pop 2 has the smallest size and the less convergence, we therefore expect that impairments in color perception after infantile strabismus may be more difficult to rehabilitate through convergence and interaction processes than the perception of the other attributes. However, interactions between the two populations Pop 1 and Pop 2 in $V_{1}$ and visual processing in superior visual areas could compensate for this (cf. Table 2).

As developed in section Data Which Support Our New Perspective, our hypothesis is already supported by published data. These results could have impact for future. However, only four attributes of the visual scene are presently really considered: the classical ones (visual acuity, i.e., spatial frequency, binocularity, i.e., eye alignment and stereopsis, i.e., $3 \mathrm{D}$ perception) and also the contrast. We hypothesize that other (if not all) attributes may also be involved, i.e., may improve by themselves but it is not currently known. Therefore, firstly, it would be of interest to examine this with appropriate psychophysical tests. For example, it would be informative to establish that the perception of velocity and direction of movement have also been improved in each situation described in the previous paragraph after rehabilitation of infantile amblyopia/strabismus. If not, developing specific tools to rehabilitate and/or stimulate perception of movement/direction could lead to improve global visual perception in cases of infantile strabismus. Improving spatial location could also be beneficial. New and appropriate perceptual training programs would also need to be developed. This is also relevant for the other visual attributes. This would be challenging but, if the results are as we expect, this may have great interest for patients with infantile strabismus, whatever their age.

Furthermore, we have shown that postural stability may also be improved when visual perception is improved after infantile strabismus. This indicates that the functionality of other systems which differ from the visual one but which are vision-dependent may also be improved when visual perception is improved. Promising data and the development of new therapeutic strategies already favor such new perspectives (cf. section Data Which Support Our New Perspective).

Note that: (a) our hypothesis applies to infantile strabismus with either early-onset (developing within the first 24 months of birth) or late-onset, with the limitation that rehabilitation of binocular vision and thus of 3D perception may not be expected in the former case; (b) in principle, our hypothesis may apply to all forms of infantile strabismus (convergent, divergent ...); (c) the simultaneous rehabilitation of different visual attributes has been already obtained both in children and adults with infantile strabismus. This is consistent with our hypothesis which considers that the visual system is essentially organized the same way in both cases. This is also consistent with brain capacity to "learn" throughout life including in adulthood (e.g., Buonomano and Merzenich, 1998; Gilbert et al., 2001, 2009; Sales et al., 2011), and even has the capacity of modifying the functional specializations of visual cortical areas (Adab et al., 2014; Chen et al., 2016). However, our expectation is that rehabilitation may be faster and/or even more extensive in youngest subjects because of a higher plasticity, in particular during the global critical period; (d) each visual attribute has its own critical period with its own time course (cf. Introduction and section Principle of Convergence in Visual Cortex). Thus, to optimize the rehabilitative processes discussed here, it would seem pertinent to, when possible, take advantage of the optimal plasticity that exists during each critical period, for each visual attribute. Unfortunately, this is presently difficult given the time course of the critical period is known for only two visual attributes (binocular vision and acuity); (e) rodent models have allowed identifying neuro-chemical changes that control the onset of the critical period for binocular vision, as well as molecular and 
structural brakes that lead to the diminution of the plasticity thereafter (e.g., Hensch, 2005; Sugiyama et al., 2008, 2009; Hensch and Bilimoria, 2012; Prochiantz et al., 2014; Prochiantz and Di Nardo, 2015; Bernard and Prochiantz, 2016 for reviews). However, no application of these findings has been developed for humans yet, as far as we are aware. Any application would likely help in the present context, although the functioning of the brain is probably too complex to be adjusted simply by a drug (or a cocktail of drugs). In addition, manipulating the brain of a child during development raises ethical questions that would need to be addressed; ( $f$ ) in all cases, the eyes need to be aligned (or realigned) to ensure the success of the rehabilitation process of perception and to extend this, not only for binocular vision but also for eye movements themselves. We reiterate here that if eye movements are (still) abnormal (in case of strabismus; before and/or after treatment), visual perception and all other processes which are vision-dependent will remain altered; $(\mathrm{g})$ in rehabilitation of vision, all the visual cortical areas from $V_{1}$ and beyond need to be rehabilitated, with recognition that the former will need to be rehabilitated first (e.g., Hooks and Chan, 2007); (h) exercise and attention play major roles during the rehabilitation processes we have discussed, up to adulthood. For example, it has been demonstrated recently that voluntary physical exercise may promote ocular dominance plasticity in $\mathrm{V}_{1}$ of the adult mouse (Kalogeraki et al., 2014). It has also been shown that attention may enhance spatial resolution (Mineault et al., 2016; see also Barbot, 2016 for review); (i) during the perceptual learning training, the stimuli being used, the duration of each session, the frequency of the sessions and their total duration are of importance and need to be determined carefully. These parameters may be subject to change depending on the attributes concerned; (j) perceptive rehabilitation after the training sessions seems to have a long-term effect, through a meta-plasticity of the brain, which is evidently fundamental here (e.g., Bocci et al., 2014).

\section{REFERENCES}

Abe, H., McManus, J. N., Ramalingam, N., Li, W., Marik, S. A., Borgloh, S. M., et al. (2015). Adult cortical plasticity studied with chronically implanted electrode arrays. J. Neurosci. 35, 2778-2790. doi: 10.1523/JNEUROSCI.3579-14.2015

Adab, H. Z., Popivanov, I. D., Vanduffel, W., and Vogels, R. (2014). Perceptual learning of simple stimuli modifies stimulus representations in posterior inferior temporal cortex. J. Cogn. Neurosci. 26, 2187-2200. doi: 10.1162/jocn_a_00641

Aissani, C., Cottereau, B., Dumas, G., Paradis, A. L., and Lorenceau,. J. (2011). Magnetoencephalographic signatures of visual form and motion binding. Brain Res. 1408, 27-40. doi: 10.1016/j.brainres.2011.05.051

Aissani, C., Martinerie, J., Yahia-Cherif, L., Paradis, A. L., and Lorenceau, J. (2014). Beta, but not gamma, band oscillations index visual form-motion integration. PLoS ONE 9:e95541. doi: 10.1371/journal.pone.0095541

Albrecht, D. G., and De Valois, R. L. (1981). Striate cortex responses to periodic patterns with and without the fundamental harmonics. J. Physiol. 319, 497-514. doi: 10.1113/jphysiol.1981.sp013922

Albrecht, D. G., De Valois, R. L., and Thorell, L. G. (1980). Visual cortical neurons: are bars or gratings the optimal stimuli? Science 207, 88-90

Allman, J., Miezin, F., and McGuinness, E. (1985). Direction- and velocityspecific responses from beyond the classical receptive field in the middle
To conclude, we propose approaches to improve rehabilitation after infantile strabismus and some suggestions for their implementation. Some data from fundamental research already support these views. However, further work remains to be carried out. To proceed, tools already in existence and those under development will need to be validated in order to be systematically available for medical practitioners. A multidisciplinary approach will also be required. A deeper cooperation between medical and paramedical practitioners from different domains will be essential. The body is indeed a "multi-sensorial" and a "sensory-motor" system and not a "mono-sensorial" one. A more profound working relationship between practitioners and neuroscientists will also be essential to allow a rapid transfer of fundamental knowledge to practice. The end result will be of great benefit to patients.

\section{AUTHOR CONTRIBUTIONS}

Study concept and design: CM. Neural bases for visual perception, psychophysical and modeling data about vision and postural stability (normal condition and after strabismus): CM. Epidemiology, etiology and treatments of strabismus: EB. Critical revision of the manuscript: $\mathrm{CM}$ and $\mathrm{EB}$.

\section{ACKNOWLEDGMENTS}

We would like to thank the Group LUZ- OPTIKID and the Group OPTIQUE DE BOURBON (Reunion Island, France) for their financial supports, Sidney Wiener and Chloe Ann Barker for discussions and comments on the manuscript, France Maloumian for assistance in the preparation of the figures; Frontiers in Integrative Neuroscience and Elsevier Masson for permissions to reproduce Figures 2, 5, 6 respectively. temporal visual area (MT). Perception 14, 105-126. doi: 10.1068/p 140105

Alotaibi, A. Z., Alghadir, A., Iqbal, Z. A., and Anwer, S. (2016). Effect of absence of vision on posture. J. Phys. Ther. Sci. 28, 1374-1377. doi: 10.1589/jpts.28.1374

An, X., Gong, H., Yin, J., Wang, X., Pan, Y., Zhang, X., et al. (2014). Orientation-cue invariant population responses to contrast-modulated and phase-reversed contour stimuli in macaque V1 and V2. PLoS ONE 9:e106753. doi: 10.1371/journal.pone. 0106753

Angelaki, D. E., Gu, Y., and Deangelis, G. C. (2011). Visual and vestibular cue integration for heading perception in extrastriate visual cortex. J. Physiol. 589(Pt 4), 825-833. doi: 10.1113/jphysiol.2010.194720

Angelucci, A., and Bressloff, P. C. (2006). Contribution of feed-forward, lateral and feedback connections to the classical receptive field center and extra-classical receptive field surround of primate V1 neurons. Prog. Brain Res. 154, 93-120. doi: 10.1016/S0079-6123(06)54005-1

Angelucci, A., Levitt, J. B., Walton, E. J., Hupe, J. M., Bullier, J., and Lund, J. S. (2002). Circuits for local and global signal integration in primary visual cortex. J. Neurosci. 22, 8633-8646. doi: 10.1523/JNEUROSCI.22-1908633.2002

Angelucci, A., and Rosa, M. G. (2015). Resolving the organization of the third tier visual cortex in primates: a hypothesis-based approach. Vis. Neurosci. 32:E010. doi: $10.1017 /$ S0952523815000073 
Anzai, A., Ohzawa, I., and Freeman, R. D. (1997). Neural mechanisms underlying binocular fusion and stereopsis: position vs. phase. Proc. Natl. Acad. Sci. U.S.A. 94, 5438-5443. doi: 10.1073/pnas.94.10.5438

Arcaro, M. J., Pinsk, M. A., and Kastner, S. (2015). The anatomical and functional organization of the human visual pulvinar. J. Neurosci. 35, 9848-9871. doi: 10.1523/JNEUROSCI.1575-14.2015

Ashton, J. A., Boddy, A., Dean, S. R., Milleret, C., and Donaldson, I. M. (1988). Afferent signals from cat extraocular muscles in the medial vestibular nucleus, the nucleus praepositus hypoglossi and adjacent brainstem structures. Neuroscience 26, 131-145. doi: 10.1016/0306-4522(88) 90132-7

Ashton, J. A., Milleret, C., and Donaldson, I. M. (1989). Effects of afferent signals from the extraocular muscles upon units in the cerebellum, vestibular nuclear complex and oculomotor nucleus of the trout. Neuroscience 31, 529-541. doi: 10.1016/0306-4522(89)90395-3

Baker, D. H., Meese, T. S., Mansouri, B., and Hess, R. F. (2007). Binocular summation of contrast remains intact in strabismic amblyopia. Invest. Ophthalmol. Vis. Sci. 48, 5332-5338. doi: 10.1167/iovs.07-0194

Banks, M. S., Aslin, R. N., and Letson, R. D. (1975). Sensitive period for the development of human binocular vision. Science 190, 675-677.

Barbot, A. (2016). How attention enhances spatial resolution: preferential gain enhancement of high spatial frequency neurons. J. Neurosci. 36, 12080-12082. doi: 10.1523/JNEUROSCI.2691-16.2016.

Barnes, G. R., Hess, R. F., Dumoulin, S. O., Achtman, R. L., and Pike, G. B. (2001). The cortical deficit in humans with strabismic amblyopia. J. Physiol. 533(Pt 1), 281-297. doi: 10.1111/j.1469-7793.2001.0281b.x

Baron, J. (1950). Musculature extrinsèque et équilibre des Poissons. C.R. Acad. Sci. (Paris), 230, 2231-2233.

Baroncelli, L., Maffei, L., and Sale, A. (2011). New perspectives in amblyopia therapy on adults: a critical role for the excitatory/inhibitory balance. Front. Cell Neurosci. 5:25. doi: 10.3389/fncel.2011.00025

Batardiere, A., Barone, P., Dehay, C., and Kennedy, H. (1998). Area-specific laminar distribution of cortical feedback neurons projecting to cat area 17: quantitative analysis in the adult and during ontogeny. J. Comp. Neurol. 396, 493-510. doi: 10.1002/(SICI)1096-9861(19980713)396:4\&lt;493::AIDCNE6\&gt;3.0.CO;2-X

Bartels, A., and Zeki, S. (2000). The architecture of the colour centre in the human visual brain: new results and a review. Eur. J. Neurosci. 12, 172-193. doi: 10.1046/j.1460-9568.2000.00905.x

Bartfeld, E., and Grinvald, A. (1992). Relationships between orientation-preference pinwheels, cytochrome oxidase blobs, and ocular-dominance columns in primate striate cortex. Proc. Natl. Acad. Sci. U.S.A. 89, 11905-11909. doi: 10.1073/pnas.89.24.11905

Basole, A., Kreft-Kerekes, V., White, L. E., and Fitzpatrick, D. (2006). Cortical cartography revisited: a frequency perspective on the functional architecture of visual cortex. Prog. Brain Res. 154, 121-134. doi: 10.1016/S0079-6123(06)54006-3

Basole, A., White, L. E., and Fitzpatrick, D. (2003). Mapping multiple features in the population response of visual cortex. Nature 423, 986-990. doi: $10.1038 /$ nature 01721

Berardi, N., Bisti, S., Cattaneo, A., Fiorentini, A., and Maffei, L. (1982). Correlation between the preferred orientation and spatial frequency of neurones in visual areas 17 and 18 of the cat. J. Physiol. 323, 603-618. doi: 10.1113/jphysiol.1982.sp014094

Berman, N., and Daw, N. W. (1977). Comparison of the critical periods for monocular and directional deprivation in cats. J. Physiol. 265, 249-259. doi: 10.1113/jphysiol.1977.sp011715

Berman, R. A., and Wurtz, R. H. (2008). Exploring the pulvinar path to visual cortex. Prog. Brain Res. 171, 467-473. doi: 10.1016/S0079-6123(08)00668-7

Berman, R. A., and Wurtz, R. H. (2011). Signals conveyed in the Pulvinar pathway from the superior colliculus to cortical area MT. J. Neurosci. 31, 373-384. doi: 10.1523/JNEUROSCI.4738-10.2011.

Bernard, C., and Prochiantz, A. (2016). Otx2-PNN Interaction to Regulate Cortical Plasticity. Neural Plast. 2016, 7931693. doi: 10.1155/2016/7931693

Birch, E. E. (2013). Amblyopia and binocular vision. Prog. Retin. Eye Res. 33, 67-84. doi: 10.1016/j.preteyeres.2012.11.001

Bisti, S., Carmignoto, G., Galli, L., and Maffei, L. (1985). Spatialfrequency characteristics of neurones of area 18 in the cat: dependence on the velocity of the visual stimulus. J. Physiol. 359, 259-268. doi: 10.1113/jphysiol.1985.sp015584

Black, J. M., Hess, R. F., Cooperstock, J. R., To, L., and Thompson, B. (2012). The measurement and treatment of suppression in amblyopia. J. Vis. Exp. 70:e3927. doi: $10.3791 / 3927$

Blakemore, C., and Tobin, E. A. (1972). Lateral inhibition between orientation detectors in the cat's visual cortex. Exp. Brain Res. 15, 439-440.

Blasdel, G. G., and Salama, G. (1986). Voltage-sensitive dyes reveal a modular organization in monkey striate cortex. Nature 321, 579-585. doi: $10.1038 / 321579 \mathrm{a} 0$

Blaser, E., Papathomas, T., and Vidnyánszky, Z. (2005). Binding of motion and colour is early and automatic. Eur. J. Neurosci. 21, 2040-2044. doi: 10.1111/j.1460-9568.2005.04032.x

Bocci, T., Caleo, M., Tognazzi, S., Francini, N., Briscese, L., Maffei, L., et al. (2014). Evidence for metaplasticity in the human visual cortex. J. Neural. Transm. 121, 221-231. doi: 10.1007/s00702-013-1104-z

Bolz, J., and Gilbert, C. D. (1986). Generation of end-inhibition in the visual cortex via interlaminar connections. Nature 320, 362-365. doi: 10.1038/320 $362 \mathrm{a} 0$

Bonhoeffer, T., and Grinvald, A. (1991). Iso-orientation domains in cat visual cortex are arranged in pinwheel-like patterns. Nature 353, 429-431. doi: $10.1038 / 353429 \mathrm{a} 0$

Bonhoeffer, T., and Grinvald, A. (1993a). Optical imaging of the functional architecture in cat visual cortex: the layout of direction and orientation domains. Adv. Exp. Med. Biol. 333, 57-69. doi: 10.1007/978-1-4899-2468-1_7

Bonhoeffer, T., and Grinvald, A. (1993b). The layout of isoorientation domains in area 18 of cat visual cortex: optical imaging reveals a pinwheel-like organization. J. Neurosci. 13, 4157-4180. doi: 10.1523/JNEUROSCI.13-10-04157.1993

Bonhoeffer, T., Kim, D. S., Malonek, D., Shoham, D., and Grinvald, A. (1995). Optical imaging of the layout of functional domains in area 17 and across the area $17 / 18$ border in cat visual cortex. Eur. J. Neurosci. 7, 1973-1988. doi: 10.1111/j.1460-9568.1995.tb00720.x

Bosking, W. H., Kretz, R., Pucak, M. L., and Fitzpatrick, D. (2000). Functional specificity of callosal connections in tree shrew striate cortex. J. Neurosci. 20, 2346-2359. doi: 10.1523/JNEUROSCI.20-06-02346.2000

Bosking, W. H., Zhang, Y., Schofield, B., and Fitzpatrick, D. (1997). Orientation selectivity and the arrangement of horizontal connections in tree shrew striate cortex. J. Neurosci. 17, 2112-2127. doi: 10.1523/JNEUROSCI.17-06-02112.1997

Bridge, H., Leopold, D. A., and Bourne, J. A. (2016). Adaptive pulvinar circuitry supports visual cognition. Trends Cogn. Sci. 20, 146-157. doi: 10.1016/j.tics.2015.10.003

Bringuier, V., Chavane, F., Glaeser, L., and Frégnac, Y. (1999). Horizontal propagation of visual activity in the synaptic integration field of area 17 neurons. Science 283, 695-699. doi: 10.1126/science.283.5402.695

Britten, K. H. (2008). Mechanisms of self-motion perception. Annu. Rev. Neurosci. 31, 389-410. doi: 10.1146/annurev.neuro.29.051605.112953

Bucci, M. P., Brémont-Gignac, D., and Kapoula, Z. (2009). Speed and accuracy of saccades, vergence and combined eye movements in subjects with strabismus before and after eye surgery. Vision Res. 49, 460-469. doi: 10.1016/j.visres.2008.12006.

Bucci, M. P., Kapoula, Z., Yang, Q., Roussat, B., and Brémont-Gignac, D. (2002). Binocular coordination of saccades in children with strabismus before and after surgery. Invest. Ophthalmol. Vis. Sci., 43, 1040-1047.

Bucci, M. P., Soufi, H., Villeneuve, P., Collevielle, L., Bui Quoc, E., and Lions, C. (2016). Importance of proprioceptive information for postural control in children with strabismus before and after strabismus surgery. Front. Syst. Neurosci. 10:67. doi: 10.3389/fnsys.2016.00067

Bui Quoc, E., and Milleret, C. (2014). Origins of strabismus and loss of binocular vision. Front. Integr. Neurosci. 8:71. doi: 10.3389/fnint. 2014.00071

Bui Quoc, E., Ribot, J., Quenech'Du, N., Doutremer, S., Lebas, N., Grantyn, A., et al. (2012). Asymmetrical interhemispheric connections develop in cat visual cortex after early unilateral convergent strabismus: anatomy, physiology and mechanisms. Front. Neuroan. 5:68. doi: 10.3389/fnana.2011.00068

Buisseret, P. (1995). Influence of extraocular muscle proprioception on vision. Physiol. Rev. 75, 323-338.

Buonomano, D. V., and Merzenich, M. M. (1998). Cortical plasticity: from synapses to maps. Annu. Rev. Neurosci. 21, 149-186. 
Burge, J., and Geisler, W. S. (2015). Optimal speed estimation in natural image movies predicts human performance. Nat. Commun. 6, 7900. doi: $10.1038 /$ ncomms 8900

Buzás, P., Eysel, U. T., Adorján, P., and Kisvárday, Z. F. (2001). Axonal topography of cortical basket cells in relation to orientation, direction, and ocular dominance maps. J. Comp. Neurol. 437, 259-285. doi: 10.1002/cne.1282

Buzás, P., Volgushev, M., Eysel, U. T., and Kisvárday, Z. F. (2003). Independence of visuotopic representation and orientation map in the visual cortex of the cat. Eur. J. Neurosci. 18, 957-968. doi: 10.1046/j.1460-9568.2003.02808.x

Caclin, A., Paradis, A. L., Lamirel, C., Thirion, B., Artiges, E., Poline, J. B., et al. (2012). Perceptual alternations between unbound moving contours and bound shape motion engage a ventral/dorsal interplay. J. Vis. 12:11. doi: $10.1167 / 12.7 .11$

Campbell, F. W., and Maffei, L. (1981). The influence of spatial frequency and contrast on the perception of moving patterns. Vision Res. 21, 713-721 doi: 10.1016/0042-6989(81)90080-8

Carandini, M., and Sengpiel, F. (2004). Contrast invariance of functional maps in cat primary visual cortex. J. Vis. 4, 130-143. doi: 10.1167/4.3.1

Casanova, C. (2004). "The visual functions of the pulvinar," in The Visual Neurosciences, eds L. M. Chalupa and J. S (Cambridge: Werner The MIT Press), 592-608.

Casanova, C., Merabet, L., Desautels, A., and Minville, K. (2001). Higherorder motion processing in the pulvinar. Prog. Brain Res. 134, 71-82. doi: 10.1016/S0079-6123(01)34006-2

Cattan, S., Bachatene, L., Bharmauria, V., Jeyabalaratnam, J., Milleret, C., and Molotchnikoff, S. (2014). Comparative analysis of orientation maps in areas 17 and 18 of the cat primary visual cortex following adaptation. Eur. J Neurosci. 40, 2554-2563. doi: 10.1111/ejn.12616

Chapman, B., Stryker, M. P., and Bonhoeffer, T. (1996). Development of orientation preference maps in ferret primary visual cortex. J. Neurosci. 16, 6443-6453. doi: 10.1523/JNEUROSCI.16-20-06443.1996

Chen, N., Cai, P., Zhou, T., Thompson, B., and Fang, F. (2016). Perceptual learning modifies the functional specializations of visual cortical areas. Proc. Natl. Acad. Sci. U.S.A. 113, 5724-5729 doi: 10.1073/pnas.1524160113

Chino, Y. M., Shansky, M. S., Jankowski, W. L., and Banser, F. A. (1983). Effects of rearing kittens with convergent strabismus on development of receptive field properties in striate cortex neurons. J. Neurophysiol. 50, 265-286. doi: 10.1152/jn.1983.50.1.265

Chino, Y. M., Smith, E. L., Yoshida, K., Cheng, H., and Hamamoto, J. (1994). Binocular interactions in striate cortical neurons of cats reared with discordant visual inputs. J. Neurosci. 14, 5050-5067. doi: 10.1523/JNEUROSCI.14-08-05050.1994

Chisum, H. J., Mooser, F., and Fitzpatrick, D. (2003). Emergent properties of layer $2 / 3$ neurons reflect the collinear arrangement of horizontal connections in tree shrew visual cortex. J. Neurosci. 23, 2947-2960. doi: 10.1523/JNEUROSCI.23-07-02947.2003

Clavagnier, S., Dumoulin, S. O., and Hess, R. F. (2015). Is the cortical deficit in amblyopia due to reduced cortical magnification, loss of neural resolution, or neural disorganization? J. Neurosci. 35, 14740-14755. doi: 10.1523/JNEUROSCI.1101-15.2015

Clavagnier, S., Thompson, B., and Hess, R. F. (2013). Long lasting effects of daily theta burst rTMS sessions in the human amblyopic cortex. Brain Stimul. 6, 860-867. doi: 10.1016/j.brs.2013.04.002

Clifford, C. W., Spehar, B., Solomon, S. G., Martin, P. R., and Zaidi, Q. (2003). Interactions between color and luminance in the perception of orientation. J. Vis. 3, 106-115. doi: 10.1167/3.2.1

Cloutman, L. L. (2013). Interaction between dorsal and ventral processing streams: where, when and how? Brain Lang. 127, 251-263. doi: 10.1016/j.bandl.2012.08.003

Collins, J. A., and Olson, I. R. (2014). Beyond the FFA: the role of the ventral anterior temporal lobes in face processing. Neuropsychologia. 61, 65-79. doi: 10.1016/j.neuropsychologia.2014.06.005

Connor, C. E., Brincat, S. L., and Pasupathy, A. (2007). Transformation of shape information in the ventral pathway. Curr. Opin. Neurobiol. 17, 140-147. doi: 10.1016/j.conb.2007.03.002

Conway, B. R. (2009). Color vision, cones, and color-coding in the cortex. Neuroscientist 15, 274-290. doi: 10.1177/1073858408331369
Conway, B. R. (2014). Color signals through dorsal and ventral visual pathways Vis. Neurosci. 31, 197-209. doi: 10.1017/S0952523813000382

Costa, T. L., Hamer, R. D., Nagy, B. V., Barboni, M. T., Gualtieri, M., Boggio, P. S., et al. (2015). Transcranial direct current stimulation can selectively affect different processing channels in human visual cortex. Exp. Brain Res. 233, 1213-1223. doi: 10.1007/s00221-015-4199-7

Cowey, A., Stoerig, P., and Bannister, M. (1994). Retinal ganglion cells labelled from the pulvinar nucleus in macaque monkeys. Neuroscience 61, 691-705. doi: 10.1016/0306-4522(94)90445-6

Crair, M. C., Gillespie, D. C., and Stryker, M. P. (1998). The role of visual experience in the development of columns in cat visual cortex. Science 279, 566-570.

Crair, M. C., Ruthazer, E. S., Gillespie, D. C., and Stryker, M. P. (1997). Ocular dominance peaks at pinwheel center singularities of the orientation map in cat visual cortex. J. Neurophysiol. 77, 3381-3385. doi: 10.1152/jn.1997.77.6.3381

Crawford, M. L., and Harwerth, R. S. (2004). Ocular dominance column width and contrast sensitivity in monkeys reared with strabismus or anisométropie. Invest. Ophthalmol. Vis. Sci. 45, 3036-3042. doi: 10.1167/iovs.04-0029

Crowley, J. C., and Katz, L. C. (1999). Development of ocular dominance columns in the absence of retinal input. Nat. Neurosci. 2, 1125-1130. doi: 10.1038/16051

Das, A., and Gilbert, C. D. (1997). Distortions of visuotopic map match orientation singularities in primary visual cortex. Nature 387, 94-598. doi: 10.1038/ 42461

Davis, A. R., Sloper, J. J., Neveu, M. M., Hogg, C. R., Morgan, M. J., and Holder, G. E. (2008). Differential changes in color and motion-onset visual evoked potentials from both eyes in early- and late-onset strabismic amblyopia. Invest. Ophthalmol. Vis. Sci. 49, 4418-4426. doi: 10.1167/iovs.07-1437

Daw, N. W. (1998). Critical periods and amblyopia. Arch. Ophthalmol. 116, 502-504.

Daw, N. W. (2009). Critical periods: motion sensitivity is early in all areas. Curr. Biol. 19, R336-R338. doi: 10.1016/j.cub.2009.02.048

Daw, N. W., Berman, N. E., and Ariel, M. (1978). Interaction of critical periods in the visual cortex of kittens. Science 199, 565-567. doi: 10.1126/science.622560

DeAngelis, G. C., Freeman, R. D., and Ohzawa, I. (1994). Length and width tuning of neurons in the cat's primary visual cortex. J. Neurophysiol. 71, 347-374. doi: 10.1152/jn.1994.71.1.347

DeAngelis, G. C., Ohzawa, I., and Freeman, R. D. (1991). Depth is encoded in the visual cortex by a specialized receptive field structure. Nature 352, 156-159. doi: $10.1038 / 352156 \mathrm{a} 0$

Deco, G., Ponce-Alvarez, A., Hagmann, P., Romani, G. L., Mantini, D., and Corbetta, M. (2014). How local excitation-inhibition ratio impacts the whole brain dynamics. J. Neurosci. 34, 7886-7898. doi: 10.1523/JNEUROSCI.5068-13.2014

Donaldson, I. M. (2000). The functions of the proprioceptors of the eye muscles. Philos. Trans. R. Soc. Lond. B 355, 1685-1754. doi: 10.1098/rstb.2000.0732

Dong, Y., Mihalas, S., Qiu, F., von der Heydt, R., and Niebur, E. (2008), Synchrony and the binding problem in macaque visual cortex. J. Vision 8, 1-16. doi: $10.1167 / 8.7 .30$

Dragoi, V., Rivadulla, C., and Sur, M. (2001). Foci of orientation plasticity in visual cortex. Nature 411, 80-86. doi: 10.1038/35075070

Dragoi, V., Sharma, J., and Sur, M. (2000). Adaptation-induced plasticity of orientation tuning in adult visual cortex. Neuron 28, 287-298. doi: 10.1016/S0896-6273(00)00103-3

Duan, Y., Norcia, A. M., Yeatman, J. D., and Mezer, A. (2015). The structural properties of major white matter tracts in strabismic amblyopia. Invest. Ophthalmol. Vis. Sci. 56, 5152-5160. doi: 10.1167/iovs.15-17097

Dumbrava, D., Faubert, J., and Casanova, C. (2001). Global motion integration in the cat's lateral posterior-Pulvinar complex. Eur. J. Neurol. 13, 2218-2226. doi: 10.1046/j.0953-816x.2001.01598.x

Durand, J. B., Celebrini, S., and Trotter, Y. (2007). Neural bases of stereopsis across visual field of the alert macaque monkey. Cereb. Cortex 17, 1260-1273. doi: 10.1093/cercor/bhl050

Durand, J. B., Girard, P., Barone, P., Bullier, J., and Nowak, L. G. (2012). Effects of contrast and contrast adaptation on static receptive field features in macaque area V1. J. Neurophysiol. 108, 2033-2050. doi: 10.1152/jn.00936.2011

Eber, A. M., Strubel-Streicher, D., Guillot, M., and Collard, M. (1984). Loss of balance, vertigo and nystagmus induced by maximum excentration of gaze. The role of extraocular proprioception. Rev. Neurol. 140, 131-137. 
Economides, J. R., Adams, D. L., and Horton, J. C. (2012). Perception via the deviated eye in strabismus. J Neurosci. 32, 10286-10295. doi: 10.1523/JNEUROSCI.1435-12.2012

Engel, A. K., Fries, P., and Singer, W. (2001). Dynamic predictions: oscillations and synchrony in top-down processing. Nature Rev. 2, 704-716. doi: 10.1038/35094565

Engel, A. K., König, P., Kreiter, A. K., and Singer, W. (1991). Stimulus-dependent neuronaloscillations in cat visual cortex: inter-columnar interactions as determined by cross-correlation analysis. Eur. J. Neurosci. 2, 588-606. doi: 10.1111/j.1460-9568. 1990.tb00449

Engelmann, R., Crook, J. M., and Löwel, S. (2002). Optical imaging of orientation and ocular dominance maps in area 17 of cats with convergent strabismus. Vis. Neurosci. 19, 39-49. doi: 10.1017/S0952523801191042

Enroth-Cugell, C., and Robson, J. G. (1966). The contrast sensitivity of retinal ganglion cells of the cat. J. Physiol. 187, 517-552. doi: 10.1113/jphysiol.1966.sp008107

Epelbaum, M., Milleret, C., Buisseret, P., and Dufier, J. L. (1993). The sensitive period for strabismic amblyopia in man. Ophthalmology $100 \mathrm{~N}^{\circ} 3,323-327$. doi: 10.1016/S0161-6420(13)32170-8

Espinosa, J. S., and Stryker, M. P. (2012). Development and plasticity of the primary visual cortex. Neuron 75, 230-249. doi: 10.1016/j.neuron.2012. 06.009

Ezane, M. D., Lions, C., Bui Quoc, E., Milleret, C., and Bucci, M. P. (2015). Spatial and temporal analyzes of posture in strabismic children. Graefes Arch. Clin. Exp. Ophthalmol. 253, 1629-1639. doi: 10.1007/s00417-0153134-8

Farley, B. J., Yu, H., Jin, D. Z., and Sur, M. (2007). Alteration of visual input results in a coordinated reorganization of multiple visual cortex maps. J. Neurosci. 27, 10299-10310. doi: 10.1523/JNEUROSCI.2257-07.2007

Federer, F., Ichida, J. M., Jeffs, J., Schiessl, I., McLoughlin, N., and Angelucci, A. (2009). Four projection streams from primate V1 to the cytochrome oxidase stripes of V2. J. Neurosci. 29, 15455-15471. doi: 10.1523/JNEUROSCI.1648-09.2009.

Felleman, D. J., and Van Essen, D. C. (1991). Distributed hierarchical processing in the primate cerebral cortex. Cereb. Cortex 1, 1-47. doi: 10.1093/cercor/1.1.1

Ferrera, V. P., Rudolph, K. K., and Maunsell, J. H. (1994). Responses of neurons in the parietal and temporal visual pathways during a motion task. J. Neurosci. 14, 6171-6186. doi: 10.1523/JNEUROSCI.14-10-06171.1994

Fong, M. F., Mitchell, D. E., Duffy, K. R., and Bear, M. F. (2016). Rapid recovery from the effects of early monocular deprivation is enabled by temporary inactivation of the retinas. Proc. Natl. Acad. Sci. U.S.A. 113, 14139-14144. doi: $10.1073 /$ pnas. 1613279113

Frégnac, Y., Bringuier, V., Chavane, F., Glaeser, L., and Lorenceau, J. (1996). An intracellular study of space and time representation in primary visual cortical receptive fields. J. Physiol. 90, 189-197. doi: 10.1016/S0928-4257(97)81422-2

Frégnac, Y., and Imbert, M. (1978). Early development of visual cortical cells in normal and dark-reared kittens: relationship between orientation selectivity and ocular dominance. J. Physiol. 278, 27-44. doi: 10.1113/jphysiol.1978.sp012290

Fries, P. (2005). A mechanism for cognitive dynamics: neuronal communication through neuronal coherence. Trends Cogn. Sci. 9, 474-480. doi: 10.1016/j.tics.2005.08.011

Fries, P., Schröder, J. H., Roelfsema, P. R., Singer, W., and Engel, A. K. (2002). Oscillatory neuronal synchronization in primary visual cortex as a correlate of stimulus selection. J. Neurosci. 22, 3739-3754. doi: 10.1523/JNEUROSCI.22-09-03739.2002

Frostig, R. D., Lieke, E. E., Ts'o, D. Y., and Grinvald, A. (1990). Cortical functional architecture and local coupling between neuronal activity and the microcirculation revealed by in vivo high-resolution optical imaging of intrinsic signals. Proc. Natl. Acad. Sci. U.S.A. 87, 6082-6086. doi: $10.1073 /$ pnas.87.16.6082

Gaertner, C., Creux, C., Espinasse-Berrod, M. A., Orssaud, C., Dufier, J. L., and Kapoula, Z. (2013). Benefit of bi-ocular visual stimulation for postural control in children with strabismus. PLOS ONE 8:e60341. doi: 10.1371/journal.pone.0060341.

Gagey, P. M., Baron, J. B., Lespargot, J., and Poli, J. P. (1973). Variations de l'activité tonique posturale et activité des muscles oculocéphalogyres en cathédrostatisme. Agressologie 14B, 87-95.
Galuske, R. A., Schmidt, K. E., Goebel, R., Lomber, S. G., and Payne, B. R. (2002) The role of feedback in shaping neural representations in cat visual cortex. Proc. Natl. Acad. Sci. U.S.A. 99, 17083-17088. doi: 10.1073/pnas.242399199

Gamberini, M., Bakola, S., Passarelli, L., Burman, K. J., Rosa, M. G. P., Fattori, P., et al. (2016). Thalamic projections to visual and visuo-motor areas (V6 and V6A) in the Rostral Bank of the parieto-occipital sulcus of the Macaque. Brain Struct. Funct. 221, 1573-1589. doi: 10.1007/s00429-015-0990-2

Garey, L. J., and de Courten, C. (1983). Structural development of the lateral geniculate nucleus and visual cortex in monkey and man. Behav. Brain Res. 10, 3-13.

Garraghty, P. E., Roe, A. W., Chino, Y. M., and Sur, M. (1989). Effects of convergent strabismus on the development of physiologically identified retinogeniculate axons in cats. J. Comp. Neurol. 289, 202-212. doi: 10.1002/cne.902890203

Gegenfurtner, K. R., Kiper, D. C., and Levitt, J. B. (1997). Functional properties of neurons in macaque area V3. J. Neurophysiol. 77, 1906-1923. doi: 10.1152/jn.1997.77.4.1906

Georges, S., Seriès, P., Frégnac, Y., and Lorenceau, J. (2002). Orientation dependent modulation of apparent speed: psychophysical evidence. Vision Res. 42, 2757-2772. doi: 10.1016/S0042-6989(02)00303-6

Gerard-Mercier, F., Carelli, P. V., Pananceau, M., Troncoso, X. G., and Frégnac, Y. (2016). Synaptic correlates of low-level perception in V1. J. Neurosci. 36, 3925-3942. doi: 10.1523/JNEUROSCI.4492-15.2016

Ghisovan, N., Nemri, A., Shumikhina, S., and Molotchnikoff, S. (2008). Synchrony between orientation-selective neurons is modulated during adaptation-induced plasticity in cat visual cortex. BMC Neurosci. 9:60. doi: 10.1186/1471-2202-9-60

Ghisovan, N., Nemri, A., Shumikhina, S., and Molotchnikoff, S. (2009). Long adaptation reveals mostly attractive shifts of orientation tuning in cat primary visual cortex. Neuroscience 164, 1274-1283. doi: 10.1016/j.neuroscience.2009.09.003

Gilaie-Dotan, S., Saygin, A. P., Lorenzi, L. J., Egan, R., Rees, G., and Behrmann, M. (2013). The role of human ventral visual cortex in motion perception. Brain. 136(Pt 9), 2784-2798. doi: 10.1093/brain/awt214

Gilbert, C. D. (1983). Microcircuitry of the visual cortex. Annu. Rev. Neurosci. 6, 217-247.

Gilbert, C. D., Das, A., Ito, M., Kapadia, M., and Westheimer, G. (1996). Spatial integration and cortical dynamics. Proc. Natl. Acad. Sci. U.S.A. 93, 615-622. doi: $10.1073 /$ pnas.93.2.615

Gilbert, C. D., and Li, W. (2013). Top-down influences on visual processing. Nat. Rev. Neurosci. 14, 350-363. doi: 10.1038/nrn3476

Gilbert, C. D., Li, W., and Piech, V. (2009). Perceptual learning and adult cortical plasticity. J. Physiol. 587, 2743-2751. doi: 10.1113/jphysiol.2009.171488

Gilbert, C. D., Sigman, M., and Crist, R. E. (2001). The neural basis of perceptual learning. Neuron 31, 681-697. doi: 10.1016/S0896-6273(01)00424-X

Gilbert, C. D., and Wiesel, T. N. (1983). Clustered intrinsic connections in cat visual cortex. J. Neurosci. 3, 1116-1133. doi: 10.1523/JNEUROSCI.03-05-01116.1983

Gilbert, C. D., and Wiesel, T. N. (1989). Columnar specificity of intrinsic horizontal and cortico-cortical connections in cat visual cortex. J. Neurosci. 9, 2432-2442. doi: 10.1523/JNEUROSCI.09-07-02432.1989

Gilbert, C. D., and Wiesel, T. N. (1990). The influence of contextual stimuli on the orientation selectivity of cells in primary visual cortex of the cat. Vision Res. 30, 1689-1701. doi: 10.1016/0042-6989(90)90153-C

Gligorović, M., Vuvcinić, V., Eškirović, B., and Jablan, B. (2011). The influence of manifest strabismus and stereoscopic vision on non-verbal abilities of visually impaired children. Res. Dev. Disabil. 32, 1852-1859. doi: $10.1016 /$ j.ridd.2011.03.018

Godde, B., Leonhardt, R., Cords, S. M., and Dinse, H. R. (2002). Plasticity of orientation preference maps in the visual cortex of adult cats. Proc. Natl. Acad. Sci. U.S.A. 99, 6352-6357. doi: 10.1073/pnas.082407499

Goodale, M. A., Króliczak, G., and Westwood, D. A. (2005). Dual routes to action: contributions of the dorsal and ventral streams to adaptive behavior. Prog. Brain Res. 149, 269-283. doi: 10.1016/S0079-6123(05)49019-6

Goodyear, B. G., Nicolle, D. A., and Menon, R. S. (2002). High resolution fMRI of ocular dominance columns within the visual cortex of human amblyopes. Strabismus. 10, 129-136. doi: 10.1076/stra.10.2.129.8140

Gray, C. M., Koenig, P., Engel, A. K., and Singer, W. (1989). Oscillatory responses in cat visual cortex exhibit inter-columnar synchronization which reflects global stimulus properties. Nature 23, 334-337. doi: 10.1038/338334a0 
Greenlee, M. W. (2000). Human cortical areas underlying the perception of optic flow: brain imaging studies. Int. Rev. Neurobiol. 44, 269-292. doi: 10.1016/S0074-7742(08)60746-1

Grefkes, C., and Fink, G. R. (2005). The functional organization of the intraparietal sulcus in humans and monkeys. J. Anat. 207, 3-17. doi: 10.1111/j.1469-7580.2005.00426.x

Grieve, K. L., Acuña, C., and Cudeiro, J. (2000). The primate pulvinar nuclei: vision and action. Trends Neurosci. 23, 35-39. doi: 10.1016/S0166-2236(99)01482-4

Grinvald, A., Frostig, R. D., Siegel, R. M., and Bartfeld, E. (1991). High-resolution optical imaging of functional brain architecture in the awake monkey. Proc. Natl. Acad. Sci. U.S.A. 88, 11559-11563. doi: 10.1073/pnas.88.24.11559

Gross, C. G. (2008). Single neuron studies of inferior temporal cortex. Neuropsychologia 46, 841-852. doi: 10.1016/j.neuropsychologia.2007.11.009

Guerraz, M., and Bronstein, A. M. (2008). Ocular versus extraocular control of posture and equilibrium. Neurophysiol. Clin. 38, 391-398. doi: 10.1016/j.neucli.2008.09.007

Guo, K., Benson, P. J., and Blakemore, C. (2004). Pattern motion is present in V1 of awake but not anaesthetized monkeys. Eur. J. Neurosci. 19, 1055-1066. doi: $10.1111 / \mathrm{j} .1460-9568.2004 .03212 . x$

Hamm, L. M., Black, J., Dai, S., and Thompson, B. (2014). Global processing in amblyopia: a review. Front. Psychol. 5:583. doi: 10.3389/fpsyg.2014.00583

Handa, T., Ishikawa, H., Nishimoto, H., Goseki, T., Ichibe, Y., Ichibe, H., et al. (2010). Effect of motion stimulation without changing binocular disparity on stereopsis in strabismus patients. Am. Orthopt. J. 60, 87-94. doi: $10.3368 /$ aoj.60.1.87

Harwerth, R. S., Smith, E. L., Duncan, G. C., Crawford, M. L. J., and Von Norden, G. K. (1986). Multiple sensitive periods in the development of the primate visual system. Science 232, 235-238. doi: 10.1126/science.3952507

Hendry, S. H., and Reid, R. C. (2000). The koniocellular pathway in primate vision. Annu. Rev. Neurosci. 23, 127-153. doi: 10.1146/annurev.neuro.23.1.127

Hendry, S. H., and Yoshioka, T. (1994). A neurochemically distinct third channel in the macaque dorsal lateral geniculate nucleus. Science 264, 575-577. doi: $10.1126 /$ science. 8160015

Hensch, T. K. (2005). Critical period plasticity in local cortical circuits. Nat. Rev. Neurosci. 6, 877-888. doi: 10.1038/nrn1787

Hensch, T. K., and Bilimoria, P. M. (2012). Re-opening windows: manipulating critical periods for brain development. Cerebrum. 2012:11.

Hess, R. F., Babu, R. J., Clavagnier, S., Black, J., Bodier, W., and Thompson, B. (2014b). The iPod binocular home- based treatment for amblyopia in adults: efficacy and compliance. Clin. Exp. Opt. 97, 389-398. doi: 10.1111/cxo.12192.

Hess, R., and Field, D. (1999). Integration of contours: new insights. Trends Cogn. Sci. 3, 480-486.

Hess, R. F., Li, X., Mansouri, B., Thompson, B., and Hansen, B. C. (2009). Selectivity as well as sensitivity loss characterizes the cortical spatial frequency deficit in amblyopia. Hum. Brain Mapp. 30, 4054-4069. doi: $10.1002 / \mathrm{hbm} .20829$

Hess, R. F., Mansouri, B., and Thompson, B. (2010a). A new binocular approach to the treatment of amblyopia in adults well beyond the critical period of visual development. Restor. Neurol. Neurosci. 28, 793-802. doi: 10.3233/RNN-2010-0550

Hess, R. F., Mansouri, B., and Thompson, B. (2010b). A binocular approach to treating amblyopia: anti suppression therapy. Optom. Vis. Sci. 87, 697-704. doi: 10.1097/OPX.0b013e3181ea18e9

Hess, R. F., Mansouri, B., and Thompson, B. (2011). Restoration of binocular vision in amblyopia. Strabismus 19, 110-118. doi: 10.3109/09273972.2011.600418

Hess, R. F., and Thompson, B. (2013). New insights into amblyopia: binocular therapy and noninvasive brain stimulation. J. AAPOS 17, 89-93. doi: 10.1016/j.jaapos.2012.10.018

Hess, R. F., and Thompson, B. (2015). Amblyopia and the binocular approach to its therapy. Vision Res. 114, 4-16. doi: 10.1016/j.visres.2015.02.009

Hess, R. F.,Thompson, B., and Baker, D. H. (2014a). Binocular vision in amblyopia: structure, suppression and plasticity. Ophthalmic Physiol. Opt. 34, 146-162. doi: $10.1111 /$ opo. 12123

Hipp, J. F., Engel, A. K., and Siegel, M. (2011). Oscillatory synchronization in large-scale cortical networks predicst perception. Neuron 69, 387-396. doi: 10.1016/j.neuron.2010.12.027

Ho, C. S., and Giaschi, D. E. (2006). Deficient maximum motion displacement in amblyopia. Vision Res. 46, 4595-4603. doi: 10.1016/j.visres.2006.09.025
Ho, C. S., and Giaschi, D. E. (2009). Low- and high-level motion perception deficits in anisometropic and strabismic amblyopia: evidence from fMRI. Vision Res. 49, 2891-2901. doi: 10.1016/j.visres.2009.07.012

Ho, C. S., Paul, P. S., Asirvatham, A., Cavanagh, P., Cline, R., and Giaschi, D. E. (2006). Abnormal spatial selection and tracking in children with amblyopia. Vision Res. 46, 3274-3283. doi: 10.1016/j.visres.2006.03.029

Holmin, J., and Nawrot, M. (2015). Motion parallax thresholds for unambiguous depth perception. Vision Res. $115(\mathrm{Pt}$ A), 40-47. doi: $10.1016 /$ j.visres.2015.07.002

Hooks, B. M., and Chan, C. (2007). Critical periods in the visual system: changing views for a model of experience-dependent plasticity. Neuron 56, 312-326. doi: 10.1016/j.neuron.2007.10.003

Horak, F. B. (2010). Postural compensation for vestibular loss and implications for rehabilitation. Restor. Neurol. Neurosci.28, 57-68. doi: 10.3233/RNN-2010-0515

Houzel, J. C., Milleret, C., and Innocenti, G. (1994). Morphology of callosal axons interconnecting areas 17 and 18 of the cat. Eur. J. Neurosci. 6, 898-917.

Huang, P. C., Li, J., Deng, D., Yu, M., and Hess, R. F. (2012). Temporal synchrony deficits in amblyopia. Invest. Ophthal. Vis. Sci. 53, 8325-8332. doi: $10.1167 /$ iovs.12-10835

HUBEL, D. H., and WIESEL, T. N. (1962). Receptive fields, binocular interaction and functional architecture in the cat's visual cortex. J. Physiol. 160, 106-154. doi: 10.1113/jphysiol.1962.sp006837

Hubel, D. H., and Wiesel, T. N. (1963a). Shape and arrangement of columns in cat 's striate cortex. J. Physiol. 165, 559-568.

HUBEL, D. H., and WIESEL, T. N. (1963b). Receptive fields of cells in striate cortex of very young, visually inexperienced kittens. J. Neurophysiol. 26, 994-1002.

Hubel, D. H., and Wiesel, T. N. (1965). Receptive fields and functional architecture in two non striate visual areas (18 and 19) of the cat. J. Neurophysiol. 28, 229-289. doi: 10.1152/jn.1965.28.2.229

Hubel, D. H., and Wiesel, T. N. (1969). Anatomical demonstration of columns in the monkey striate cortex. Nature 1221, 747-750. doi: 10.1038/221747a0

Hubel, D. H., and Wiesel, T. N. (1970). The period of susceptibility to the physiological effects of unilateral eye closure in kittens. J. Physiol. 206, 419-436. doi: 10.1113/jphysiol.1970.sp009022

Hubel, D. H., and Wiesel, T. N. (1988). Receptive fields and functional architecture of monkey striate cortex. J. Physiol. 195, 215-243. doi: 10.1113/jphysiol.1968.sp008455

Hubel, D. H., Wiesel, T. N., and Stryker, M. P. (1978). Anatomical demonstration of orientation columns in macaque monkey. J. Comp. Neurol. 177, 361-380.

Hübener, M., Shoham, D., Grinvald, A., and Bonhoeffer, T. (1997). Spatial relationships among three columnar systems in cat area 17. J. Neurosci. 17, 9270-9284. doi: 10.1523/JNEUROSCI.17-23-092 70.1997

Husk, J. S., Farivar, R., and Hess, R. F. (2012). Amblyopic deficits in processing structure-from-motion. J. Vis. 12:4. doi: 10.1167/12.4.4

Husk, J. S., and Hess, R. F. (2013). Global processing of orientation in amblyopia. Vision Res. 82, 22-30. doi: 10.1016/j.visres.2013.02.005

Innocenti, G. M. (1986). "General organization of callosal connections in the cerebral cortex," in Cerebral Cortex. Vol. 5., eds E. G. Jones and A. Peters (New York, NY: Plenum Press), 291-354.

Innocenti, G. M., and Frost, D. O. (1979). Effects of visual experience on the maturation of the efferent system to the corpus callosum. Nature 280, 231-234.

Isotalo, E., Kapoula, Z., Feret, P. H., Gauchon, K., Zamfirescu, F., and Gagey, P. M. (2004). Monocular versus binocular vision in postural control. Auris. Nasus Larynx. 31, 11-17. doi: 10.1016/j.anl.2003.10.001

Issa, N. P., Trepel, C., and Stryker, M. P. (2000). Spatial frequency maps in cat visual cortex. J. Neurosci. 20, 8504-8514. doi: 10.1523/JNEUROSCI.20-22-08504.2000

Iwaki, S., Bonmassar, G., and Belliveau, J. W. (2013). Dynamic cortical activity during the perception of three-dimensional object shape from two-dimensional random-dot motion. J. Integr. Neurosci. 12, 355-367. doi: 10.1142/S0219635213500210

Kalil, R. E., Spear, P. D., and Langsetmo, A. (1984). Response properties of striate cortex neurons in cats raised with divergent or convergent strabismus. J. Neurophysiol. 52, 514-537. doi: 10.1152/jn.1984.52.3.514

Kalogeraki, E., Greifzu, F., Haack, F., and Löwel, S. (2014). Voluntary physical exercise promotes ocular dominance plasticity in adult mouse primary visual cortex. J. Neurosci. 34, 15476-15481. doi: 10.1523/JNEUROSCI.2678-14.2014 
Kapadia, M. K., Ito, M., Gilbert, C. D., and Westheimer, G. (1995). Improvement in visual sensitivity by changes in local context: parallel studies in human observers and in V1 of alert monkeys. Neuron 15, 843-856. doi: 10.1016/0896-6273(95)90175-2

Kara, P., and Boyd, J. D. (2009). A micro-architecture for binocular disparity and ocular dominance in visual cortex. Nature 458, 627-631. doi: 10.1038 /nature07721

Keech, R. V., and Kutschke, P. J. (1995). Upper age limit for the development of amblyopia. J. Pediatr. Ophthalmol. Strabismus. 32, 89-93.

Keizer, A. W., Colzato, L. S., and Hommel, B. (2008). Integrating faces, houses, motion, and action: spontaneous binding across ventral and dorsal processing streams. Acta Psychol. 127, 177-185. doi: 10.1016/j.actpsy.2007.04.003

Kenet, T., Bibitchkov, D., Tsodyks, M., Grinvald, A., and Arieli, A. (2003). Spontaneously emerging cortical representations of visual attributes. Nature 425, 954-956. doi: 10.1038/nature02078

Kim, D. S., Matsuda, Y., Ohki, K., Ajima, A., and Tanaka, S. (1999). Geometrical and topological relationships between multiple functional maps in cat primary visual cortex. Neuroreport 10, 2515-2522. doi: 10.1097/00001756-199908200-00015

Kimiskidis, V. K., Valentin, A., and Kälviäinen, R. (2014). Transcranial magnetic stimulation for the diagnosis and treatment of epilepsy. Curr. Opin. Neurol. 27, 236-241. doi: 10.1097/WCO.0000000000000071.

Kiorpes, L. (2006). Visual processing in amblyopia: animal studies. Strabismus 14, 3-10. doi: 10.1080/09273970500536193

Kiorpes, L. (2015). Visual development in primates: neural mechanisms and critical periods. Dev. Neurobiol. 75, 1080-1090. doi: 10.1002/dneu.22276

Kiorpes, L., Kiper, D. C., and Movshon, J. A. (1993). Contrast sensitivity and vernier acuity in amblyopic monkeys. Vision Res. 33, 2301-2311. doi: 10.1016/0042-6989(93)90107-8

Kiorpes, L., Kiper, D. C., O’Keefe, L. P., Cavanaugh, J. R., and Movshon, J. A. (1998). Neuronal correlates of amblyopia in the visual cortex of macaque monkeys with experimental strabismus and anisométropie. J. Neuroosci. 18, 6411-6424.

Kiorpes, L., and McKee, S. P. (1999). Neural mechanisms underlying amblyopia. Curr. Opin. Neurobiol. 9, 480-486.

Kisvárday, Z. F. (2016). “Topography of excitatory cortico-cortical connections in three main tiers of the visual cortex: functional implications of the patchy horizontal network," in Axon and Brain architecture, ed K. S. Rockland (Elsevier Academic Press), Chapter 7, 135-158. doi: 10.1016/B978-0-12-801393-9.00007-4

Kisvárday, Z. F., Buzás, P., and Eysel, U. T. (2001). Calculating direction maps from intrinsic signals revealed by optical imaging. Cereb. Cortex 11, 636-647. doi: 10.1093/cercor/11.7.636

Kisvárday, Z. F., Crook, J. M., Buzás, P., and Eysel, U. T. (2000). Combined physiological-anatomical approaches to study lateral inhibition. J. Neurosci. Methods. 103, 91-106. doi: 10.1016/S0165-0270(00)00299-5

Klainguti, G. (2005). Early surgery in infantile esotropia. Klin. Monbl. Augenheilkd. 222, 172-174. doi: 10.1055/s-2005-857974

Koçak-Altintas, A. G., Satana, B., Koçak, I., and Duman, S. (2000). Visual acuity and color vision deficiency in amblyopia. Eur. J. Ophthalmol. 10, 77-81. doi: $10.1177 / 112067210001000113$

Koch, G. (2013). Do studies on cortical plasticity provide a rationale for using noninvasive brain stimulation as a treatment for Parkinson's disease patients? Front. Neurol. 4:180. doi: 10.3389/fneur.2013.00180

Koshino, H., Carpenter, P. A., Keller, T. A., and Just, M. A. (2005). Interactions between the dorsal and the ventral pathways in mental rotation: an fMRI study. Cogn. Affect. Behav. Neurosci. 5, 54-66. doi: 10.3758/CABN.5.1.54

Kravitz, D. J., Saleem, K. S., Baker, C. I., and Mishkin, M. (2011). A new neural framework for visuospatial processing. Nat. Rev. Neurosci. 12, 217-230. doi: $10.1038 / \mathrm{nrn} 3008$

Kravitz, D. J., Saleem, K. S., Baker, C. I., Ungerleider, L. G., and Mishkin, M. (2013). The ventral visual pathway: an expanded neural framework for the processing of object quality. Trends Cogn. Sci. 17, 26-49. doi: 10.1016/j.tics.2012.10.011

Kremkow, J., Jin, J., Wang, Y., and Alonso, J. M. (2016). Principles underlying sensory map topography in primary visual cortex. Nature 533, 52-57. doi: $10.1038 /$ nature 17936

Kuffler, S. W. (1953). Discharge patterns and functional organization of mammalian retina. J. Neurophysiol. 16, 37-68
Kwan, W. C., Mundinano, I. C., de Souza, M. J., Lee, S. C. S., Martin, P. R., Grünert, U., et al. (2018). Unravelling the subcortical and retinal circuitry of the primate inferior pulvinar. J. Comp. Neurol. doi: 10.1002/cne.24387. [Epub ahead of print].

Lalanne, C., and Lorenceau, J. (2006). Directional shifts in the barber pole illusion: effects of spatial frequency, spatial adaptation, and lateral masking. Vis. Neurosci. 23, 729-739. doi: 10.1017/S0952523806230050

Landisman, C. E., and Ts'o, D. Y. (2002). Color processing in macaque striate cortex: relationships to ocular dominance, cytochrome oxidase, and orientation. J. Neurophysiol. 87, 3126-3137 doi: 10.1152/jn.2002.87.6.3126

Lee, K. S., Huang, X., and Fitzpatrick, D. (2016). Topology of ON and OFF inputs in visual cortex enables an invariant columnar architecture. Nature 533, 90-94. doi: 10.1038/nature17941

Legrand, A., Bui-Quoc, E., and Bucci, M. P. (2012). Re-alignment of the eyes, with prisms and with eye surgery, affects postural stability differently in children with strabismus. Graefes Arch. Clin. Exp. Ophthalmol. 250, 849-855. doi: 10.1007/s00417-011-1845-Z

Legrand, A., Bui Quoc, E., Vacher, S. W., Ribot, J., Lebas, N., Milleret, C., et al. (2011). Postural control in children with strabismus: effect of eye surgery. Neurosci. Lett. 501, 96-101. doi: 10.1016/j.neulet.2011.06.056

Leguire, L. E., Rogers, G. L., and Bremer, D. L. (1991). Visual-evoked response binocular summation in normal and strabismic infants. Defining the critical period. Invest. Ophthalmol. Vis. Sci. 32, 126-133.

Lehky, S. R., and Tanaka, K. (2016). Neural representation for object recognition in inferotemporal cortex. Curr. Opin. Neurobiol. 37, 23-35. doi: 10.1016/j.conb.2015.12.001

LeVay, S., Connolly, M., Houde,. J., and Van Essen, D. C. (1985). The complete pattern of ocular dominance stripes in the striate cortex and visual field of the macaque monkey. J. Neurosci. 5, 486-501. doi: 10.1523/JNEUROSCI.05-02-00486.1985

Levi, D. M., Knill, D. C., and Bavelier, D. (2015). Stereopsis and amblyopia: a mini-review. Vision Res. 114, 17-30. doi: 10.1016/j.visres.2015.01.002

Levitt, J. B., and Lund, J. S. (1997). Contrast dependence of contextual effects in primate visual cortex. Nature 387, 73-76. doi: 10.1038/387073a0

Lewis, T. L., and Maurer, D. (2005). Multiple sensitive periods in human visual development: evidence from visually deprived children. Dev. Psychobiol. 46, 163-183. doi: 10.1002/dev.20055

Li, J., Spiegel, D. P., Hess, R. F., Chen, Z., Chan, L. Y., Deng, D., et al. (2015). Dichoptic training improves contrast sensitivity in adults with amblyopia. Vision Res. 114, 161-172. doi: 10.1016/j.visres.2015.01.017

Li, R., Polat, U., Makous, W., and Bavelier, D. (2009). Enhancing the contrast sensitivity function through action video game training. Nat. Neurosci. 12, 549-551. doi: 10.1038/nn.2296

Li, X., Mullen, K. T., Thompson, B., and Hess, R. F. (2011). Effective connectivity anomalies in human amblyopia. Neuroimage 54, 505-516. doi: 10.1016/j.neuroimage.2010.07.053

Li, J., Thompson, B., Deng, D., Chan, L. Y., Yu, M., and Hess, R. F. (2013). Dichoptic training enables the adult amblyopic brain to learn. Curr.Biol. 23, R308-R309. doi: 10.1016/j.cub.2013.01.059

Li, Y., Fitzpatrick, D., and White, L. (2006). The development of direction selectivity in ferret visual cortex requires early visual experience. Nat. Neurosci. 9, 676-681. doi: 10.1038/nn1684

Li, Y., Van Hooser, S. D., Mazurek, M., White, L. E., and Fitzpatrick, D. (2008). Experience with moving visual stimuli drives the early development of cortical direction selectivity. Nature 456, 952-956. doi: 10.1038/nature07417

Linares, D., and López-Moliner, J. (2006). Perceptual asynchrony between color and motion with a single direction change. J. Vis. 6, 974-981. doi: $10.1167 / 6.9 .10$

Lions, C., Bui-Quoc, E., Wiener-Vacher, S., and Bucci, M. P. (2014). Effect of proprioceptive information during postural control in strabismic children. Front. Physiol. 5:156. doi: 10.3389/fphys.2014.00156

Lions, C., Colleville, L., Bui-Quoc, E., and Bucci, M. P. (2016). Importance of visual quality for postural stability in strabismic children. Neurosci. Lett. 617, 127-133. doi: 10.1016/j.neulet.2016.02.008

Livingstone, M. S., and Hubel, D. H. (1984a). Anatomy and physiology of a color system in the primate visual cortex. J. Neurosci. 4, 309-356.

Livingstone, M. S., and Hubel, D. H. (1984b). Specificity of intrinsic connections in primate primary visual cortex. J. Neurosci. 4, 2830-2835. 
Livingstone, M. S., and Hubel, D. H. (1988). Segregation of form, color, motion and depth: anatomy, physiology and perception. Science 240, 740-749. doi: 10.1126/science.3283936.

Löwel, S. (1994). Ocular dominance columns development: strabismus changes the spacing of adjacent columns in cat visual cortex. J. Neurosci. 14, 7451-7468. doi: 10.1523/JNEUROSCI.14-12-07451.1994

Löwel, S., Schmidt, K. E., Kim, D. S., Wolf, F., Hoffsümmer, F., Singer, W., et al. (1998). The layout of orientation and ocular dominance domains in area17 of strabismic cats. Eur. J. Neurosci. 10, 2629-2643. doi: 10.1046/j.1460-9568.1998.00274.x

Löwel, S., and Singer, W. (1987). The pattern of ocular dominance columns in flat-mounts of the cat visual cortex. Exp. Brain Res. 68, 661-666.

Löwel, S., and Singer, W. (1992). Selection of intrinsic horizontal connections in the visual cortex by correlated neuronal activity. Science 255, 209-212. doi: $10.1126 /$ science. 1372754

Lu, H. D., and Roe, A. W. (2007). Optical imaging of contrast response in macaque monkey V1 and V2. Cereb. Cortex 17, 2675-2695. doi: 10.1093/cercor/bhl177

Lu, H. D., and Roe, A. W. (2008). Functional organization of color domains in V1 and V2 of macaque monkey revealed by optical imaging. Cereb. Cortex 18, 516-533. doi: 10.1093/cercor/bhm081

Lund, R. D., and Mitchell, D. E. (1979). Asymmetry in the visual callosal connections of strabismic cats. Brain Res. 167, 176-179. doi: 10.1016/0006-8993(79)90274-9

Maffei, L., Morrone, C., and Pirchio, M. (1979). Responses of visual cortical cells to periodic and non-periodic stimuli. J. Physiol. 296, 27-47. doi: 10.1113/jphysiol.1979.sp012989

Maldonado, P. E., Gödecke, I., Gray, C. M., and Bonhoeffer, T. (1997). Orientation selectivity in pinwheel centers in cat striate cortex. Science 276, 1551-1555. doi: 10.1126/science.276.5318.1551

Mansouri, B., and Hess, R. F. (2006). The global processing deficit in amblyopia involves noise segregation. Vis. Res. 46, 4104-4117. doi: 10.1016/j.visres.2006.07.017

Mansouri, B., Singh, P., Globa, A., and Pearson, P. (2014). Binocular training reduces amblyopic visual acuity impairment. Strabismus 22, 1-6. doi: 10.3109/09273972.2013.877945

Mansouri, B.,Thompson, B., and Hess, R. F. (2008). Measurement of suprathresold old binocular interactions in amblyopia. Vis.Res. 48, 2775-2784. doi: 10.1016/j.visres.2008.09.002

Mante, V., and Carandini, M. (2005). Mapping of stimulus energy in primary visual cortex. J. Neurophysiol. 94, 788-798. doi: 10.1152/jn.01094.2004

Markov, N. T., Vezoli, J., Chameau, P., Falchier, A., Quilodran, R., Huissoud, C., et al. (2014). Anatomy of hierarchy: feedforward and feedback pathways in macaque visual cortex. J. Comp. Neurol. 522, 225-259. doi: 10.1002/cne. 23458

Martin, K. A., Roth, S., and Rusch, E. S. (2014). Superficial layer pyramidal cells communicate heterogeneously between multiple functional domains of cat primary visual cortex. Nat. Commun. 5, 5252. doi: 10.1038/ ncomms6252

Marucchi, C. (1987). Coordimetry of version and postural exam complement. Agressologie 28, 9a.

Marucchi, C., and Gagey, P. M. (1987). Postural blindness. Agressologie 28:9b.

Massion, J. (1994). Postural control system. Curr. Opin. Neurobiol. 4, 877-887.

Massion, J. (1997). Cerveau et Motricité: Fonctions Sensori-Motrices. Paris: Presses Universitaires.

Matsuo, T., Narita, A., Senda, M., Hasebe, S., and Ohtsuki, H. (2006). Body sway increases immediately after strabismus surgery. Acta Med. Okayama 60, 13-24.

McCarthy, J. D., Cordeiro, D., and Caplovitz, G. P. (2012). Local form-motion interactions influence global form perception. Atten. Percept. Psychophys. 74, 816-823. doi: 10.3758/s13414-012-0307-y

McMahon, D. B., and Olson, C. R. (2009). Linearly additive shape and color signals in monkey inferotemporal cortex. J. Neurophysiol. 101, 1867-1875. doi: 10.1152/jn.90650.2008.

Meier, K., Sum, B., and Giaschi, D. (2016). Global motion perception in children with amblyopia as a function of spatial and temporal stimulus parameters. Vision Res. 127, 18-27. doi: 10.1016/j.visres.2016.06.011

Merabet, L., Desautels, A., Minville, K., and Casanova, C. (1998). Motion integration in a thalamic visual nucleus. Nature 396, 265-268. doi: $10.1038 / 24382$
Merker, B. (2013). "Cortical gamma oscillations: the functional key is activation, not cognition". Neurosci. Biobehav. Rev. 37, 401-417. doi: 10.1016/j.neubiorev.2013.01.013

Milleret, C. (1988). Functional Roles of Vision, Ocular Movements and Extraocular Proprioception During Development of Visual Cortex in the Cat. Doctoral thesis in Natural Sciences, University Pierre and Marie Curie (P6), Paris, France.

Milleret, C. (1994). Physiopathology of strabismic amblyopia. Encyclo. Med. Chir. (Paris) 21-595-A-05.

Milleret, C. (2017). "Traitement physiologique de l'information visuelle" in Déficiences Visuelles; Rapport de la Société française d’ophtalmologie, eds P. Y. Robert, B. Bodaghi, C. Corbé, G. Dupeyron, F. Gérin-Roig, B. Le Bail et al. (Elsevier; Masson), 13-19.

Milleret, C., and Buser, P. (1984). Receptive field sizes and responsiveness to light in area 18 of the adult cat after chiasmotomy. Postoperative evolution; role of visual experience. Exp. Brain Res. 57, 73-81. doi: 10.1007/BF00231133

Milleret, C., and Buser, P. (1993). Reorganization processes in the visual cortex also depend on visual experience in the adult cat. Prog. Brain Res. 95, 257-269. doi: 10.1016/S0079-6123(08)60374-X

Milleret, C., Buser, P., and Watroba, L. (2005). Unilateral paralytic strabismus in the adult cat induces plastic changes in interocular disparity along the visual midline: contribution of the corpus callosum. Vis. Neurosci. 22, 325-343. doi: $10.1017 /$ S0952523805223088

Milleret, C., Gary-Bobo, E., and Buisseret, P. (1988). Comparative development of cell properties in cortical area 18 of normal and dark-reared kittens. Exp. Brain Res. 71, 8-20.

Milleret, C., and Houzel, J. C. (2001). Visual interhemispheric transfer to areas 17 and 18 in convergent strabismic cats. Eur. J. Neurosci. 13, 137-152. doi: $10.1111 /$ j.1460-9568.2001.01360.x

Milleret, C., Houzel, J. C., and Buser, P. (1994). Pattern of development of the callosal transfer of visual information to cortical areas 17 and 18 in the cat. Eur. J. Neurosci. 6, 193-202.

Milner, P. M. (1974). A model for visual shape recognition. Psychol. Rev. 81, 521-535. doi: 10.1037/h0037149

Mineault, P. J., Tring, E., Trachtenberg, J. T., and Ringach, D. L. (2016). Enhanced spatial resolution during locomotion and heightened attention in mouse primary visual cortex. J. Neurosci. 36, 6382-6392. doi: 10.1523/JNEUROSCI.0430-16.2016

Miskiewicz, A., Buffat, S., Paradis, A. L., and Lorenceau, J. (2008). Shape and motion interactions at perceptual and attentional levels during processing of structure from motion stimuli. J. Vis. 8, 17.1-14. doi: 10.1167/8.16.17

Mitchell, D. E., Kennie, J., and Kung, D. (2009). Development of global motion perception requires early postnatal exposure to pattern light. Curr. Biol. 19, 645-649. doi: 10.1016/j.cub.2009.02.038

Mitzdorf, U. (1985). Current source-density method and application in cat cerebral cortex: investigation of evoked potentials and EEG phenomena. Physiol. Rev. 65, 37-100. doi: 10.1152/physrev.1985.65.1.37

Mitzdorf, U., and Singer, W. (1978). Prominent excitatory pathways in the cat visual cortex (A 17 and A 18): a current source density analysis of electrically evoked potentials. Exp. Brain Res. 33, 371-394 doi: 10.1007/BF00235560

Mizobe, K., Polat, U., Pettet, M. W., and Kasamatsu, T. (2001). Facilitation and suppression of single striate-cell activity by spatially discrete pattern stimuli presented beyond the receptive field. Vis. Neurosci. 18, 377-391. doi: 10.1017/S0952523801183045

Mountcastle, V. B. (1997). The columnar organization of the neocortex. Brain. 120(Pt 4), 701-722. doi: 10.1093/brain/120.4.701

Moutoussis, K. (2015). The physiology and psychophysics of the color-form relationship: a review. Front. Psychol. 6:1407. doi: 10.3389/fpsyg.2015.01407

Movshon, J. A., and Van Sluyters, R. C. (1981). Visual neural development. Annu. Rev. Psychol. 32, 477-522 doi: 10.1146/annurev.ps.32.020181.002401

Nakagama, H., Tani, T., and Tanaka, S.,(2006). Theoretical and experimental studies of relationship between pinwheel centers and ocular dominance columns in the visual cortex. Neurosci. Res. 55, 370-382. doi: 10.1016/j.neures.2006.05.004

Nashner, L. M. (1976). Adapting reflexes controlling the human posture. Exp. Brain Res. 26, 59-72. doi: 10.1007/BF00235249

Nauhaus, I., Nielsen, K. J., Disney, A. A., and Callaway, E. M. (2012). Orthogonal micro-organization of orientation and spatial frequency in primate primary visual cortex. Nat. Neurosci. 15, 1683-1690. doi: 10.1038/nn.3255 
Nelson, J. I., and Frost, B. J. (1978). Orientation-selective inhibition from beyond the classic visual receptive field. Brain Res. 139, 359-365. doi: 10.1016/0006-8993(78)90937-X

Nemri, A., Ghisovan, N., Shumikhina, S., and Molotchnikoff, S. (2009). Adaptive behavior of neighboring neurons during adaptationinduced plasticity of orientation tuning in VI. BMC Neurosci. 10:147. doi: 10.1186/1471-2202-10-147

Ohayon, S., Freiwald, W. A., and Tsao, D. Y. (2012). What makes a cell face selective? The importance of contrast. Neuron 74, 567-581. doi: 10.1016/j.neuron.2012.03.024

Ohki, K., Chung, S., Ch'ng, Y. H., Kara, P., and Reid, R. C. (2005). Functional imaging with cellular resolution reveals precise micro-architecture in visual cortex. Nature 433, 597-603. doi: 10.1038/nature03274

Ohki, K., Chung, S., Kara, P., Hübener, M., Bonhoeffer, T., and Reid, R. C. (2006). Highly ordered arrangement of single neurons in orientation pinwheels. Nature 442, 925-928. doi: 10.1038/nature05019

Ohzawa, I., DeAngelis, G. C., and Freeman, R. D. (1990). Stereoscopic depth discrimination in the visual cortex: neurons ideally suited as disparity detectors. Science 249, 1037-1041 doi: 10.1126/science.2396096

Orban, G. A. (2008). Higher order visual processing in macaque extrastriate cortex. Physiol. Rev. 88, 59-89. doi: 10.1152/physrev.00008.2007

Orban, G. A. (2011). The extraction of 3D shape in the visual system of human and nonhuman primates. Annu. Rev. Neurosci. 34, 361-388. doi: 10.1146/annurev-neuro-061010-113819

Orban, G. A., Janssen, P., and Vogels, R. (2006). Extracting 3D structure from disparity. Trends Neurosci. 29, 466-473. doi: 10.1016/j.tins.2006.06.012

Orban, G. A., Kennedy, H., and Maes, H. (1978). Influence of eccentricity on velocity characteristics of area 18 neurones in the cat. Brain Res. 159, 391-395. doi: 10.1016/0006-8993(78)90545-0

Orban, G. A., Kennedy, H., and Maes, H. (1981). Response to movement of neurons in areas 17 and 18 of the cat: velocity sensitivity. J. Neurophysiol. 45, 1043-1058. doi: 10.1152/jn.1981.45.6.1043

Paik, S. B., and Ringach, D. L. (2011). Retinal origin of orientation maps in visual cortex. Nat. Neurosci. 14, 919-925. doi: 10.1038/nn.2824

Paik, S. B., and Ringach, D. L. (2012). Link between orientation and retinotopic maps in primary visual cortex. Proc. Natl. Acad. Sci. U.S.A. 109, 7091-7096. doi: $10.1073 /$ pnas.1118926109

Paillard, J. (1971). Les déterminants moteurs de l'organisation de l'espace. Cah. Psychol. 14, 261-316.

Paillard, J. (1976). “Tonus, posture et movement," in Traité de Physiologie, Tome II. $3 r d E d n$., ed C. Kayser (Paris: Flammarion), 521-728.

Pasupathy, A. (2006). Neural basis of shape representation in the primate brain. Prog. Brain Res. 154, 293-313. doi: 10.1016/S0079-6123(06)54016-6

Paulus, W. M., Straube, A., and Brandt, T. (1984). Visual stabilization of posture. Physiological stimulus characteristics and clinical aspects. Brain. 107(Pt 4), 1143-1163. doi: 10.1093/brain/107.4.1143

Payne, B. R., and Peters, A. (2002). The Cat Primary Visual Cortex. San Diego, CA; London; New York, NY; Sydney; Tokyo; Toronto, ON: Academic Press.

Piëch, V., Li, W., Reeke, G. N., and Gilbert, C. D. (2013). Network model of topdown influences on local gain and contextual interactions in visual cortex. Proc. Natl. Acad. Sci. U.S.A. 110, E4108-E4117. doi: 10.1073/pnas.1317019110

Poggio, G. E. (1995). Mechanisms of stereopsis in monkey visual cortex. Cereb. Cortex 5, 193-204. doi: 10.1093/cercor/5.3.193

Poggio, G. F., and Poggio, T. (1984). The analysis of stereopsis. Annu. Rev. Neurosci. 7, 379-412. doi: 10.1146/annurev.ne.07.030184.002115

Polat, U., Mizobe, K., Pettet, M. W., Kasamatsu, T., and Norcia, A. M. (1998). Collinear stimuli regulate visual responses depending on cell's contrast threshold. Nature. 391, 580-584.

Pollen, D. A. (2011). On the emergence of primary visual perception. Cereb. Cortex 21, 1941-1953. doi: 10.1093/cercor/bhq285

Prochiantz, A., and Di Nardo, A. A. (2015). Homeoprotein signaling in the developing and adult nervous system. Neuron 85, 911-925. doi: 10.1016/j.neuron.2015.01.019

Prochiantz, A., Fuchs, J., and Di Nardo, A. A. (2014). Postnatal signalling with homeoprotein transcription factors. Philos. Trans. R. Soc. Lond. B. Biol. Sci. 369:20130518. doi: 10.1098/rstb.2013.0518

Rajavi, Z., Sabbaghi, H., Baghini, A. S., Yaseri, M., Sheibani, K., and Norouzi, G. (2015). Prevalence of color vision deficiency and its correlation with amblyopia and refractive errors among primary school children. J. Ophthalmic. Vis. Res. 10, 130-138. doi: 10.4103/2008-322X.163778

Ramalingam, N., McManus, J. N., Li, W., and Gilbert, C. D. (2013). Top-down modulation of lateral interactions in visual cortex. J. Neurosci. 33, 1773-1789. doi: 10.1523/JNEUROSCI.3825-12.2013

Reddy, L., and Kanwisher, N. (2006). Coding of visual objects in the ventral stream. Curr. Opin. Neurobiol. 16:40814. doi: 10.1016/j.conb.2006. 06.004

Revonsuo, A., and Newman, J. (1999). Binding and consciousness. Conscious Cogn. $8,123-127$

Ribot, J., Aushana, Y., Bui-Quoc, E., and Milleret, C. (2013). Organization and origin of spatial frequency maps in cat visual cortex. J. Neurosci. 33, 13326-13343. doi: 10.1523/JNEUROSCI.4040-12.2013

Ribot, J., Romagnoni, A., Milleret, C., Bennequin, D., and Touboul, J. (2016). Pinwheel-dipole configuration in cat early visual cortex. Neuroimage 128, 63-73. doi: 10.1016/j.neuroimage.2015.12.022

Rochefort, N. L., Buzás, P., Kisvárday, Z. F., Eysel, U. T., and Milleret, C. (2007). Layout of transcallosal activity in cat visual cortex revealed by optical imaging. Neuroimage 36, 804-821. doi: 10.1016/j.neuroimage.2007. 03.006

Rochefort, N. L., Buzás, P., Quenech'du, N., Koza, A., Eysel, U. T., Milleret, C., et al. (2009). Functional selectivity of interhemispheric connections in cat visual cortex. Cereb. Cortex 19, 2451-2465. doi: 10.1093/cercor/bhp001

Rockland, K. S., and Lund, J. S. (1983). Intrinsic laminar lattice connections in primate visual cortex. J. Comp. Neurol. 216, 303-318.

Roe, A. W., Chelazzi, L., Connor, C. E., Conway, B. R., Fujita, I., Gallant, J. L., et al. (2012). Toward a unified theory of visual area V4. Neuron 74, 12-29. doi: 10.1016/j.neuron.2012.03.011

Roelfsema, P. R., König, P., Engel, A. K., Sireteanu, R., and Singer, W. (1994). Reduced synchronization in the visual cortex of cats with strabismic amblyopia. Eur. J. Neurosci. 6, 1645-1655. doi: 10.1111/j.1460-9568

Roll, J. P., Vedel, J. P., and Roll, R. (1989). Eye, head and skeletal muscle spindle feedback in the elaboration of body references. Prog. Brain Res. 80, 113-123. discussion: 57-60.

Romagnoni, A., Ribot, J., Bennequin, D., and Touboul, J. (2015). Parsimony, exhaustivity and balanced detection in neocortex. PLoS Comput. Biol. 11:e1004623. doi: 10.1371/journal.pcbi.1004623

Rougier, P., and Lacour, M. (2006). "De Marey à nos jours: un siècle de recherche sur la posture et le mouvement," Collection Posture et Equilibre. Solal ed., 181.

Sales, A., De Pasquale, R., Bonaccorsi, J., Pietra, G., Olivieri, D., Berardi, N., et al.(2011). Visual perceptual learning induces longterm potentiation in the visual cortex. Neuroscience 172, 219-225. doi: 10.1016/j.neuroscience.2010.10.078

Sato, T. (2012). Hierarchical functional organization and face representation in inferotemporal cortex of monkeys. Brain Nerve. 64, 831-839.

Schiessl, I., and McLoughlin, N. (2003). Optical imaging of the retinotopic organization of V1 in the common marmoset. Neuroimage 20, 1857-1864. doi: 10.1016/j.neuroimage.2003.07.023

Schmidt, K. E., Kim, D. S., Singer, W., Bonhoeffer, T., and Löwel, S. (1997). Functional specificity of long-range intrinsic and interhemispheric connections in the visual cortex of strabismic cats. J. Neurosci. 17, 5480-5492. doi: 10.1523/JNEUROSCI.17-14-05480.1997

Schmidt, K. E., and Löwel, S. (2006a). The layout of functional maps in areas 18 of strabismic cats. Neuroscience 141, 1525-1531. doi: 10.1016/j.neuroscience.2006.04.056

Schmidt, K. E., and Löwel, S. (2006b). Optical imaging in cat area 18: strabismus does not enhance the segregation of ocular dominance domains. Neurimage 29, 439-445. doi: 10.1016/j.neuroimage.2005.07.031

Schmidt, K. F., and Löwel, S. (2008). Strabismus modifies intrinsic and inter-areal connections in cat area18. Neuroscience 152, 128-137. doi: 10.1016/j.neuroscience.2007.08.038

Schmidt, K. E., Singer, W., and Galuske, R. A. (2004). Processing deficits in primary visual cortex of amblyopic cats. J. Neurophysiol. 91, 1661-1671. doi: 10.1152/jn.00878.2003

Scholl, B., Tan, A. Y., and Priebe, N. J. (2013). Strabismus disrupts binocular synaptic integration in primary visual cortex. $J$. Neurosci. 33, 17108-17122. doi: 10.1523/JNEUROSCI.1831-1 3.2013 
Schuett, S., Bonhoeffer, T., and Hübener, M. (2001). Pairing-induced changes of orientation maps in cat visual cortex. Neuron 32, 325-337 doi: 10.1016/S0896-6273(01)00472-X

Schummers, J., Mariño, J., and Sur, M. (2002). Synaptic integration by V1 neurons depends on location within the orientation map. Neuron 36, 969-978. doi: 10.1016/S0896-6273(02)01012-7

Schummers, J., Sharma, J., and Sur, M. (2005). Bottom-up and topdown dynamics in visual cortex. Prog. Brain Res. 149, 65-81. doi: 10.1016/S0079-6123(05)49006-8

Schwabe, L., Obermayer, K., Angelucci, A., and Bressloff, P. C. (2006). The role of feedback in shaping the extra-classical receptive field of cortical neurons: a recurrent network model. J. Neurosci. 26, 9117-9129. doi: 10.1523/JNEUROSCI.1253-06.2006

Schwartz, J. H., Jessell, T. M., and Kandel, E. R. (2000). Principles of Neural Science, 4th End., eds E. R. Kandel, J. H. Schwartz, and T. M. Jessell. The McGraw-Hill Companies.

Sengpiel, F., and Blakemore, C. (1996). The neural basis of suppression and amblyopia in strabismus. Eye (Lond). 10(Pt 2):250-258. doi: 10.1038/eye.1996.54

Sengpiel, F., Blakemore, C., Kind, P. C., and Harrad, R. (1994). Interocular suppression in the visual cortex of strabismic cats. J. Neurosci. 14, 6855-6871. doi: 10.1523/JNEUROSCI.14-11-06855.1994

Sengpiel, F., Jirmann, K. U., Vorobyov, V., and Eysel, U. T. (2006). Strabismic suppression is mediated by inhibitory interactions in the primary visual cortex. Cereb. Cortex 16, 1750-1758. doi: 10.1093/cercor/bhj110

Sereno, A. B., and Lehky, S. R. (2011). Population coding of visual space: comparison of spatial representations in dorsal and ventral pathways. Front. Comput. Neurosci. 4:159. doi: 10.3389/fncom.2010.00159

Seriès, P., Georges, S., Lorenceau, J., and Frégnac, Y. (2002). Orientation dependent modulation of apparent speed: a model based on the dynamics of feedforward and horizontal connectivity in V1 cortex. Vision Res. 42, 2781-2797. doi: 10.1016/S0042-6989(02)00302-4

Seriès, P., Lorenceau, J., and Frégnac, Y. (2003). The "silent" surround of V1 receptive fields: theory and experiments. J. Physiol. (Paris.) 97, 453-474. doi: $10.1016 /$ j.jphysparis.2004.01.023

Shadlen, M. N., and Movshon, J. A. (1999). Synchrony unbound: a critical evaluation of the temporal binding hypothesis. Neuron 24, 67-77, 111-25. doi: 10.1016/S0896-6273(00)80822-3

Shen, L., Hu, X., Yacoub, E., and Ugurbil, K. (1999). Neural correlates of visual form and visual spatial processing. Hum. Brain Mapp. 8, 60-71. doi: 10.1002/(SICI)1097-0193(1999)8:1\&lt;60::AID-HBM5\&gt;3.0.CO;2-6

Shipp, S. (2001). Cortico-pulvinar connections of areas V5, V4, and V3 in the macaque monkey: a dual model of retinal and cortical topographies. J. Comp. Neurol. 439, 469-490. doi: 10.1002/cne.1363

Shmuel, A., and Grinvald, A. (1996). Functional organization for direction of motion and its relationship to orientation maps in cat area 18. J. Neurosci. 16, 6945-6964. doi: 10.1523/JNEUROSCI.16-21-06945.1996

Shmuel, A., and Grinvald, A. (2000). Coexistence of linear zones and pinwheels within orientation maps in cat visual cortex. Proc. Natl. Acad. Sci. U.S.A. 97, 5568-5573. doi: 10.1073/pnas.97.10.5568

Shoham, D., Hübener, M., Schulze, S., Grinvald, A., and Bonhoeffer, T. (1997). Spatio-temporal frequency domains and their relation to cytochrome oxidase staining in cat visual cortex. Nature 385, 529-533. Erratum in: Nature 1997; 386:302.

Sincich, L. C., and Horton, J. C. (2005). The circuitry of V1 and V2: integration of color, form, and motion. Annu. Rev. Neurosci. 28, 303-326. doi: $10.1146 /$ annurev.neuro.28.061604.135731

Singer, W. (1999). Neuronal synchrony: a versatile code of the definition of relations. Neuron, 24: 49-65. doi: 10.1016/S0896-6273(00)80821-1

Singer, W. (2013). Cortical dynamics revisited. Trends Congn. Sci. 17, 616-626. doi: 10.1016/j.tics.2013.09.006

Singer, W., and Gray, C. M. (1995). Visual feature integration and the temporal correlation hypothesis. Аnnu. Rev. Neurosci. 18, 555-586. doi: 10.1146/annurev.ne.18.030195.003011

Skottun, B. C. (2015). On the use of spatial frequency to isolate contributions from the magnocellular and parvocellular systems and the dorsal and ventral cortical streams. Neurosci. Biobehav. Rev. 56, 266-275. doi: $10.1016 / j . n e u b i o r e v .2015 .07 .002$
Smith, E. L., Chino, Y. M., Ni, J., Cheng, H., Crawford, M. L., and Harwerth, R. S. (1997). Residual binocular interactions in the striate cortex of monkeys reared with abnormal binocular vision. J. Neurophysiol. 78, 1353-1362. doi: 10.1152/jn.1997.78.3.1353

Smith, G. B., Sederberg, A., Elyada, Y. M., Van Hooser, S. D., Kaschube, M., and Fitzpatrick, D. (2015b). The development of cortical circuits for motion discrimination. Nat. Neurosci. 18, 252-261. doi: 10.1038/ nn. 3921

Smith, G. B., Whitney, D. E., and Fitzpatrick, D. (2015a). Modular representation of luminance polarity in the superficial layers of primary visual cortex. Neuron 88, 805-818. doi: 10.1016/j.neuron.2015.10.019

Soodak, R. E., Shapley, R. M., and Kaplan, E. (1987). Linear mechanism of orientation tuning in the retina and lateral geniculate nucleus of the cat. $J$. Neurophysiol. 58, 267-275. doi: 10.1152/jn.1987.58.2.267

Spiegel, D. P., Byblow, W. D., Hess, R. F., and Thompson, B. (2013a). Anodal transcranial direct current stimulation transiently improves contrast sensitivity and normalizes visual cortex activation in individuals with amblyopia. Neurorehabil. Neural. Repair. 27, 760-769. doi: 10.1177/1545968313 491006

Spiegel, D. P., Li, J., Hess, R. F., Byblow, W. D., Deng, D., Yu, M., et al. (2013b). Transcranial direct current stimulation enhances recovery of stereopsis in adults with amblyopia. Neurotherapeutics. 10, 831-839. doi: 10.1007/s13311-013-0200-y

Spillmann, L., Dresp-Langley, B., and Tseng, C. H. (2015). Beyond the classical receptive field: the effect of contextual stimuli. J. Vis. 15:7. doi: 10.1167/15.9.7

Spiridon, M., Fischl, B., and Kanwisher, N. (2006). Location and spatial profile of category-specific regions in human extrastriate cortex. Hum. Brain Mapp. 27, 77-89. doi: 10.1002/hbm.20169

Stettler, D. D., Das, A., Bennett, J., and Gilbert, C. D. (2002). Lateral connectivity and contextual interactions in macaque primary visual cortex. Neuron 36, 739-750. doi: 10.1016/S0896-6273(02)01029-2

Stone, J. (1983). Parallel Processing in the Visual System. The Classification of Retinal Ganglion Cells and Its Impact on the Neurobiology of Vision. Perspectives in Vision Research, ed C. Blakemore. New York, NY: Plenum Press.

Sugiyama, S., Di Nardo, A. A., Aizawa, S., Matsuo, I., Volovitch, M., Prochiantz, A., et al. (2008). Experience-dependent transfer of Otx2 homeoprotein into the visual cortex activates postnatal plasticity. Cell 134, 508-520. doi: 10.1016/j.cell.2008.05.054

Sugiyama, S., Prochiantz, A., and Hensch, T. K. (2009). From brain formation to plasticity: insights on Otx2 homeoprotein. Dev. Growth Differ. 51, 369-377. doi: 10.1111/j.1440-169X.2009.01093.x

Suzuki, M., Wolfe, J. M., Horowitz, T. S., and Noguchi, Y. (2013). Apparent colororientation bindings in the periphery can be influenced by feature binding in central vision. Vision Res. 82, 58-65. doi: 10.1016/j.visres.2013.02.011

Swindale, N. V. (1991). Coverage and the design of striate cortex. Biol Cybern. 65 , 415-424. doi: 10.1007/BF00204654

Swindale, N. V. (2000). How many maps are there in visual cortex? Cereb. Cortex 10, 633-643. doi: 10.1093/cercor/10.7.633

Swindale, N. V., Shoham, D., Grinvald, A., Bonhoeffer, T., and Hübener, M. (2000). Visual cortex maps are optimized for uniform coverage. Nat. Neurosci. 3, 822-826. doi: 10.1038/77731

Tailby, C., Dobbie, W. J., Solomon, S. G., Szmajda, B. A., Hashemi-Nezhad, M., Forte, J. D., et al. (2010). Receptive field asymmetries produce colordependent direction selectivity in primate lateral geniculate nucleus. J. Vis.10, 1. doi: $10.1167 / 10.8 .1$

Tani, T., Ribot, J., O'Hashi, K., and Tanaka, S. (2012). Parallel development of orientation maps and spatial frequency selectivity in cat visual cortex. Eur. J. Neurosci. 35, 44-55. doi: 10.1111/j.1460-9568.2011.07954.x

Taylor, J. C., and Downing, P. E. (2011). Division of labor between lateral and ventral extrastriate representations of faces, bodies, and objects. J. Cogn. Neurosci. 23, 4122-4137. doi: 10.1162/jocn_a_00091

Ten Tusscher, M. P. M., Houtman, A. C., De Mey, J., and Van Schuerbeek, P. (2018). Cortical visual connections via the corpus callosum are asymmetrical in human infantile esotropia. Strabismus 26, 22-27. doi: 10.1080/09273972.2017.1418898

Thiele, A., and Stoner, G. (2003), Neuronal synchrony does not correlate with motion coherence in cortical area MT. Nature 421, 366-370. doi: $10.1038 /$ nature 01285 
Thompson, B., Aaen-Stockdale, C. R., Mansouri, B., and Hess, R. F. (2008). Plaid perception is only subtly impaired in strabismic amblyopia. Vision Res. 48, 1307-1314. doi: 10.1016/j.visres.2008.02.020

Thompson, B., Mansouri, B., Koski, L., and Hess, R. F. (2010).From motor cortex to visual cortex: application of noninvasive brain stimulation to amblyopia. Dev. Psychobiol. 54, 263-273. doi: 10.1002/dev.20509

Thompson, B., Villeneuve, M. Y., Casanova, C., and Hess, R. F. (2012). Abnormal cortical processing of pattern motion in amblyopia: evidence from fMRI. Neuroimage 60, 1307-1315. doi: 10.1016/j.neuroimage.2012.01.078

To, L.,Thompson, B., Blum, J. R., Maehara, G., Hess, R. F., and Cooperstock, J. R. (2011). A game platform for treatment of amblyopia. IEEE Trans. Neural. Syst. Rehabil.Eng. 19, 280-289. doi: 10.1109/TNSRE.2011.2115255

Tong, L., Zhu, B., Li, Z., Shou, T., and Yu, H. (2011). Feedback from area 21a influences orientation but not direction maps in the primary visual cortex of the cat. Neurosci. Lett. 504, 141-145. doi: 10.1016/j.neulet.2011.09.019

Toth, L. J., Rao, S. C., Kim, D. S., Somers, D., and Sur, M. (1996). Subthreshold facilitation and suppression in primary visual cortex revealed by intrinsic signal imaging. Proc. Natl. Acad. Sci. U.S.A. 93, 9869-9874. doi: 10.1073 /pnas.93.18.9869

Trotter, Y., and Celebrini, S. (1999). Gaze direction controls response gain in primary visual cortex neurons. Nature 398, 239-242.

Trotter, Y., Celebrini, S., and Durand, J. B. (2004). Evidence for implication of primate area V1 in neural 3-D spatial localization processing. J. Physiol. (Paris). 98, 125-134. doi: 10.1016/j.jphysparis.2004.03.004

Trotter, Y., Celebrini, S., Stricanne, B., Thorpe, S., and Imbert, M. (1992). Modulation of neural stereoscopic processing in primate area V1 by the viewing distance. Science 257, 1279-1281.

Ts'o, D. Y., and Gilbert, C. D. (1988). The organization of chromatic and spatial interactions in the primate striate cortex. J. Neurosci. 8, 1712-1727.

Ts'o, D. Y., Frostig, R. D., Lieke, E. E., and Grinvald, A. (1990). Functional organization of primate visual cortex revealed by high resolution optical imaging. Science 249, 417-420. doi: 10.1126/science.2165630

Tsodyks, M., Kenet, T., Grinvald, A., and Arieli, A. (1999). Linking spontaneous activity of single cortical neurons and the underlying functional architecture. Science 286, 1943-1946. doi: 10.1126/science.286.5446.1943

Tusa, R. J., Palmer, L. A., and Rosenquist, A. C. (1981). "Multiple cortical visual areas: visual field topography in the cat," in Cortical Sensory Organization. 2. Multiple Visual Areas, ed C. N. Woolsey (Clifton, NJ: Humana Press), 1-31.

Tychsen, L. (2005). Can ophthalmologists repair the brain in infantile esotropia? Early surgery, stereopsis, monofixation syndrome, and the legacy of Marshall Parks. J. AAPOS. 9, 510-521. doi: 10.1016/j.jaapos.2005.06.007

Uhlhaas, P. J., Pipa, G., Neuenschwander,S., Wibral, M., and Singer, W. (2011). A new look at gamma? $(>60 \mathrm{~Hz}) \gamma$-band activity in cortical networks: function, mechanisms and impairment. Prog. Biophys. Mol. Biol. 105, 14-28. doi: 10.1016/j.pbiomolbio.2010.10.004

Uhlhaas, P. J., Roux, F., Rodriguez, E., Rotarska-Jagiela, A., and Singer, W. (2009b). Neural synchrony and the development of cortical networks. Trends Cogn. Sci. 14, 72-80. doi: 10.1016/ j.tics.2009.12.002

Uhlhaas, P. J., Roux, F., Singer, W., Haenschel, C., and Sireteanu, R. (2009a). The development of neural synchrony reflects late maturation and restructuring of functional networks in human. Proc. Natl. Acad. Sci. U.S.A. 106, 9866-9871. doi: 10.1073/pnas.0900390106

Uhlhaas, P. J., and Singer, W. (2006). Neural synchrony in brain disorders: relevance for cognitive dysfunctions and pathophysiology. Neuron 52, 155-168. doi: 10.1016/j.neuron.2006.09.020

Ungerleider, L. G., Desimone, R., Galkin, T. W., and Mishkin, M. (1984). Subcortical projections of area MT in the macaque. J. Comp. Neurol. 223, 368-386.

Ungerleider, L.G., Galkin, T.W., Desimone, R. and Gattass, R. (2008) Cortical connections of area V4 in the macaque. Cereb. Cortex. 18, 477-499. doi: $10.1093 /$ cercor/bhm061

Ungerleider, L. G., and Haxby, J. V. (1994). 'What' and 'where' in the human brain. Curr. Opin. Neurobiol. 4, 157-165. doi: 10.1016/0959-4388(94)90066-3

Ushio, N., Hinoki, M., Matsuura, K., and Baron, J. B. (1980). Role of oculomotor proprioception in the maintenance of body equilibrium; correlation with the cervical one. Agressologie, $21 \mathrm{E}: 143-152$.

Ushio, N., Matsuura, K., Hinoki, M., Baron, J. B., and Gagey, P. M. (1975). Two reflex stages in the equilibrium provoked by the proprioceptors of the oculomotor muscles. Analysis with the help of the Fukuda stepping test and of the orthostatic reflex test. Agressologie 16D, 39-52.

Vakkur, N., Bishop, P. O., and Kozak, W. (1963). Visual optics in the cat including posterior nodal distance and retinal landmarks. Vision Res. 3, 289-314. doi: 10.1016/0042-6989(63)90004-X

Van Dromme, I. C., Premereur, E., Verhoef, B. E., Vanduffel, W., and Janssen, P. (2016). Posterior parietal cortex drives infero-temporal activations during three-dimensional object vision. PLoS Biol. 14:e1002445. doi: 10.1371/journal.pbio.1002445

Van Essen, D. C. (2005). Corticocortical and thalamocortical information flow in the primate visual system. Prog. Brain Res. 149, 173-185. doi: 10.1016/S0079-6123(05)49013-5

Van Essen, D. C., and Gallant, J. L. (1994). Neural mechanisms of form and motion processing in the primate visual system. Neuron 13, 1-10. doi: 10.1016/0896-6273(94)90455-3

Vaney, D. I., Sivyer, B., and Taylor, W. R. (2012). Direction selectivity in the retina: symmetry and asymmetry in structure and function. Nat. Rev. Neurosci. 13, 194-208. doi: 10.1038/nrn3165

Vanni, M. P., and Casanova, C. (2013). Surround suppression maps in the cat primary visual cortex. Front. Neural. Circuits. 7:78. doi: 10.3389/fncir.2013.00078

Vidyasagar, T. R., and Eysel, U. T. (2015). Origins of feature selectivities and maps in the mammalian primary visual cortex. Trends Neurosci. 38, 475-485. doi: 10.1016/j.tins.2015.06.003

Villeneuve, M. Y., Kupers, R., Gjedde, A., Ptito, M., and Casanova, C. (2005). Patter-motion selectivity in the human Pulvinar. Neuroimage 28, 474-480. doi: 10.1016/j.neuroimage.2005.06.015

Vogels, R., and Orban, G. A. (1996). Coding of stimulus invariances by inferior temporal neurons. Prog. Brain Res. 112, 195-211. doi: 10.1016/S0079-6123(08)63330-0

Von der Malsburg, C. (1981). The correlation theory of brain function. MPI Biophysical Chemistry, Internal Report 81-2. Reprinted in Mod-els of Neural Networks II (1994), eds E. Domany, J.L. van Hemmen, and K. Schulten, Berlin: Springer.

Von Noorden, G. K. (1978). Application of basic research data to clinical amblyopia. Ophthalmology 85, 496-504. doi: 10.1016/S0161-6420(78)3 5652-9

Vossel, S., Geng, J. J., and Fink, G. R. (2014). Dorsal and ventral attention systems: distinct neural circuits but collaborative roles. Neuroscientist 20, 150-159. doi: $10.1177 / 1073858413494269$

Wall, M. B., and Smith, A. T. (2008). The representation of egomotion in the human brain. Curr. Biol. 18, 191-194. doi: 10.1016/j.cub.2007. 12.053

Wang, B. S., Sarnaik, R., and Cang, J. (2010). Critical period plasticity matches binocular orientation preference in the visual cortex. Neuron 65, 246-256. doi: 10.1016/j.neuron.2010.01.002

Wang, Q., Tanigawa, H., and Fujita, I. (2016). Postnatal development of intrinsic horizontal axons in macaque inferior temporal and primary visual cortices. Cereb. Cortex. 27, 2708-2726. doi: 10.1093/cercor/bhw105

Wässle, H., and Boycott, B. B. (1991). Functional architecture of the mammalian retina. Physiol. Rev. 71, 447-480.

Watanabe, I., Bi, H., Zhang, B., Sakai, E., Mori, T., Harwerth, R. S., et al. (2005). Directional bias of neurons in V1 and V2 of strabismic monkeys: temporal-to-nasal asymmetry? Invest. Ophthalmol. Vis. Sci. 46, 3899-3905. doi: 10.1167/iovs.05-0563

Watroba, L., Buser, P., and Milleret, C. (2001). Impairment of binocular vision in the adult cat induces plastic changes in the callosal cortical map. Eur. J. Neurosci. 14, 1021-1029. doi: 10.1046/j.0953-816x.2001.01720.x

Webster, M. A., and Mollon, J. D. (1997). Motion minima for different directions in color space. Vision Res. 37, 1479-1498. doi: 10.1016/S0042-6989(96)00289-1

Wei, W., and Feller, M. B. (2011). Organization and development of direction-selective circuits in the retina.Trends Neurosci. 34, 638-645. doi: 10.1016/j.tins.2011.08.002

Wenderoth, P., Watson, J. D., Egan, G. F., Tochon-Danguy, H. J., and O'Keefe, G. J. (1999). Second order components of moving plaids activate extrastriate cortex: a positron emission tomography study. NeuroImage 9, 227-234

Wiesel, T. N. (1982). Postnatal development of the visual cortex and the influence of environment. Nature 299, 583-591. doi: 10.1038/299583a0 
WIESEL, T. N., and HUBEL, D. H. (1963). Single-cell responses in striate cortex of kittens deprived of vision in one eye. J. Neurophysiol. 26, 1003-1017. doi: 10.1152/jn.1963.26.6.1003

Wiesel, T. N., and Hubel, D. H. (1966). Spatial and chromatic interactions in the lateral geniculate body of the rhesus monkey. J. Neurophysiol. 29, 1115-1156 doi: 10.1152/jn.1966.29.6.1115

Williams, M. A., Baker, C. I., Op de Beeck, H. P., Shim, W. M., Dang, S., Triantafyllou, C., et al. (2008). Feedback of visual object information to foveal retinotopic cortex. Nat. Neurosci. 11, 1439-1445. doi: 10.1038/nn.2218

Wong, A. M. (2012). New concepts concerning the neural mechanisms of amblyopia and their clinical implications. Can. J. Ophthalmol. 47, 399-409. doi: 10.1016/j.jcjo.2012.05.002

Xiao, Y. (2014). Processing of the S-cone signals in the early visual cortex of primates. Vis. Neurosci. 31, 189-195. doi: 10.1017/S0952523813000278

Yen, C. C., Fukuda, M., and Kim, S. G. (2011). BOLD responses to different temporal frequency stimuli in the lateral geniculate nucleus and visual cortex: insights into the neural basis of fMRI. Neuroimage 58, 82-90. doi: 10.1016/j.neuroimage.2011.06.022

Yinon, U. (1978). Age dependence of the effect of squint on cells in kittens visual cortex. Exp. Brain Res. 26, 151-157.

Yousef, T., Tóth, E., Rausch, M., Eysel, U. T., and Kisvárday, Z.F. (2001). Topography of orientation centre connections in the primary visual cortex of the cat. Neuroreport 12, 1693-1699. doi: 10.1097/00001756-200106130-00035

Yu, H., Farley, B. J., Jin, D. Z., and Sur, M. (2005). The coordinated mapping of visual space and response features in visual cortex. Neuron 47, 267-280. doi: 10.1016/j.neuron.2005.06.011

Yu, Q., and Shim, W. M. (2016). Modulating foveal representation can influence visual discrimination in the periphery. J. Vis. 16:15. doi: 10.1167/16.3.15
Zangenehpour, S., and Chaudhuri, A. (2005). Patchy organization and asymmetric distribution of the neural correlates of face processing in monkey inferotemporal cortex. Curr. Biol. 15, 993-1005. doi: 10.1016/j.cub.2005.04.031

Zeki, S. (1983). Colour coding in the cerebral cortex: the responses of wavelength-selective and colour-coded cells in monkey visual cortex to changes in wavelength composition. Neuroscience 9: 767-781. doi: 10.1016/0306-4522(83)90266-X

Zhang, Y., Zhang, X., Wang, Y., and Fang, F. (2016). Misbinding of color and motion in human early visual cortex: evidence from eventrelated potentials. Vision Res. 122, 51-59. doi: 10.1016/j.visres.2015. 12.010

Zhivago, K. A., and Arun, S. P. (2014). Texture discriminability in monkey inferotemporal cortex predicts human texture perception. J. Neurophysiol. 112, 2745-2755. doi: 10.1152/jn.00532.2014

Zhou, J., Jia, W., Huang, C. B., and Hess, R. F. (2012). The effects of unilateral mean luminance on binocular combination in normal and amblyopic vision. Sci. Rep. 3, 1-7. doi: 10.1038/srep02012

Conflict of Interest Statement: The authors declare that the research was conducted in the absence of any commercial or financial relationships that could be construed as a potential conflict of interest.

Copyright (C) 2018 Milleret and Bui Quoc. This is an open-access article distributed under the terms of the Creative Commons Attribution License (CC BY). The use, distribution or reproduction in other forums is permitted, provided the original author(s) and the copyright owner(s) are credited and that the original publication in this journal is cited, in accordance with accepted academic practice. No use, distribution or reproduction is permitted which does not comply with these terms. 